\title{
Scalable Simulation of Realistic Volume Fraction Red Blood Cell Flows through Vascular Networks
}

\author{
Libin $\mathrm{Lu}^{*}$ \\ Courant Institute of Mathematical \\ Sciences \\ New York University \\ New York, NY \\ libin@cs.nyu.edu
}

\author{
Matthew J. Morse* \\ Courant Institute of Mathematical \\ Sciences \\ New York University \\ New York, NY \\ mmorse@cs.nyu.edu
}

\author{
Abtin Rahimian \\ Department of Computer Science \\ University of Colorado Boulder \\ Boulder, $\mathrm{CO}$ \\ arahimian@acm.org
}

\author{
Georg Stadler \\ Courant Institute of Mathematical \\ Sciences \\ New York University \\ New York, NY \\ stadler@cims.nyu.edu
}

\author{
Denis Zorin \\ Courant Institute of Mathematical \\ Sciences \\ New York University \\ New York, NY \\ dzorin@cs.nyu.edu
}
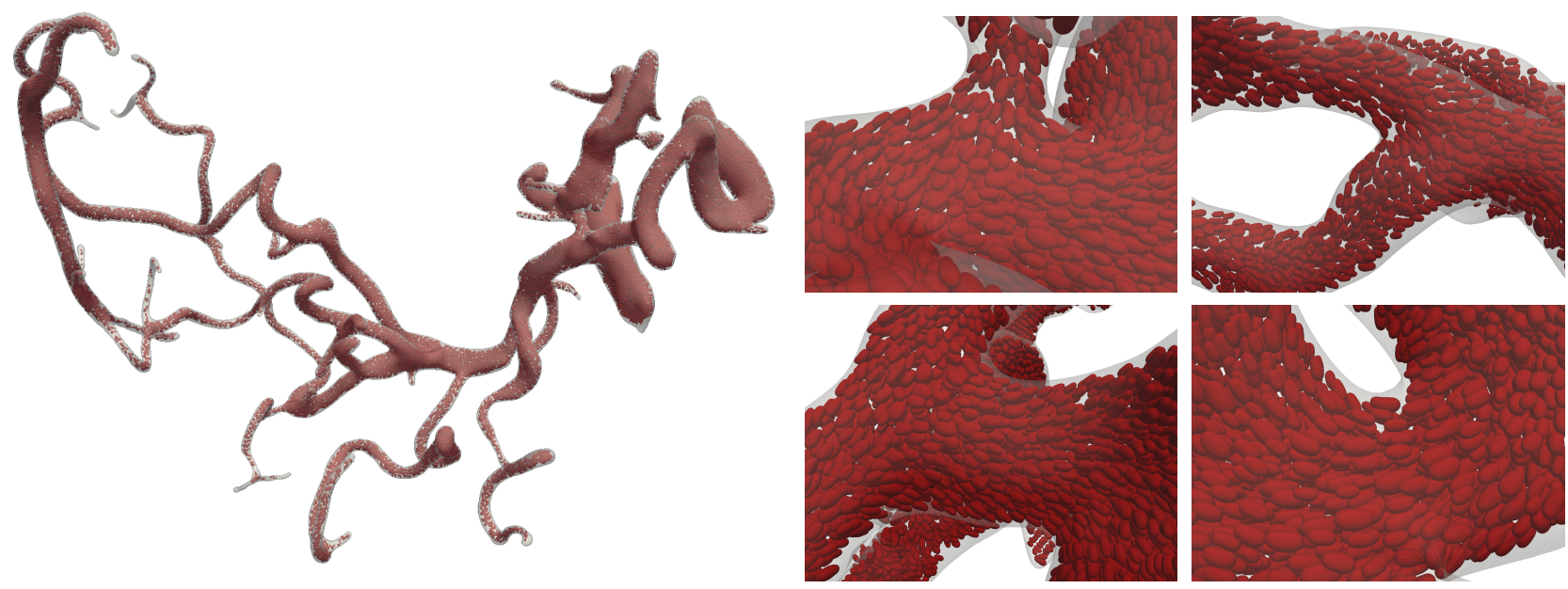

Figure 1: Simulation results for 40,960 RBCs in a complex vessel geometry. For our strong scaling experiments, we use the vessel geometry shown on the left, with inflow-outflow boundary conditions at various regions of the vessel geometry. To setup the problem, we fill the vessel with nearly-touching RBCs of different sizes. The figure above shows a setup with overall 40,960 RBCs at a volume fraction of 19\%, and 40,960 polynomial patches. The full simulation video is available at https://vimeo.com/329509229.

\begin{abstract}
High-resolution blood flow simulations have potential for developing better understanding biophysical phenomena at the microscale, such as vasodilation, vasoconstriction and overall vascular resistance. To this end, we present a scalable platform for the simulation
\end{abstract}

\footnotetext{
*Both authors contributed equally to this research.

Permission to make digital or hard copies of all or part of this work for personal or classroom use is granted without fee provided that copies are not made or distributed for profit or commercial advantage and that copies bear this notice and the full citation on the first page. Copyrights for components of this work owned by others than ACM must be honored. Abstracting with credit is permitted. To copy otherwise, or republish, to post on servers or to redistribute to lists, requires prior specific permission and/or a fee. Request permissions from permissions@acm.org.

SC '19, November 17-22, 2019, Denver, CO, USA

(C) 2019 Association for Computing Machinery.

ACM ISBN 978-1-4503-6229-0/19/11 . \$15.00

https://doi.org/10.1145/3295500.3356203
}

of red blood cell (RBC) flows through complex capillaries by modeling the physical system as a viscous fluid with immersed deformable particles. We describe a parallel boundary integral equation solver for general elliptic partial differential equations, which we apply to Stokes flow through blood vessels. We also detail a parallel collision avoiding algorithm to ensure RBCs and the blood vessel remain contact-free. We have scaled our code on Stampede 2 at the Texas Advanced Computing Center up to 34,816 cores. Our largest simulation enforces a contact-free state between four billion surface elements and solves for three billion degrees of freedom on one million RBCs and a blood vessel composed from two million patches.

\section{ACM Reference Format:}

Libin Lu, Matthew J. Morse, Abtin Rahimian, Georg Stadler, and Denis Zorin. 2019. Scalable Simulation of Realistic Volume Fraction Red Blood Cell Flows through Vascular Networks. In The International Conference for 
High Performance Computing, Networking, Storage, and Analysis (SC '19), November 17-22, 2019, Denver, CO, USA. ACM, New York, NY, USA, 14 pages. https://doi.org/10.1145/3295500.3356203

\section{INTRODUCTION}

The ability to simulate complex biological flows from first principles has the potential to provide insight into complicated physiological processes. Simulation of blood flow, in particular, is of paramount biological and clinical importance. Blood vessel constriction and dilation affects blood pressure, forces between RBCs can cause clotting, various cells migrate differently through microfluidic devices.

However, direct simulation of blood flow is an extremely challenging task. Even simulating the blood flow in smaller vessels requires modeling millions of cells (one microliter of blood contains around five million RBCs) along with a complex blood vessel. RBCs are highly deformable and cannot be well-approximated by rigid particles. The volume fraction of cells in human blood flow reaches $45 \%$, which means that a very large fraction of cells are in close contact with other cells or vessel walls at any given time. These constraints preclude a large number of discretization points per cell and make an evolving mesh of the fluid domain impractical and costly at large scale.

Simulations capable of capturing these various types of flows faithfully must be

- numerically accurate, to solve the model equations without concern for numerical error;

- robust, to handle high-volume-fraction flows, close contact between cells and vessel walls, complex geometries, and long simulation times;

- efficient and scalable, to support a realistic number of cells in flows through complex blood vessels.

Achieving these objectives for a blood flow simulation requires that the system meets a number of stringent requirements. While previous work has made significant progress [25, 28, 37], we focus on several new infrastructure components essential for handling confined flows and arbitrarily long-time, high volume fractions RBC flows; in particular, our work is able to realize each of these goals.

We formulate the viscous flow in blood vessels as an integrodifferential equation and make use of fast scalable summation algorithms for efficient implementation, as in prior RBC simulations [48]. This is the only approach to date that maintains high accuracy at the microscopic level while avoiding expensive discretization of fluid volume: all degrees of freedom reside on the surfaces of RBCs and blood vessels.

The most important novel aspects of our system include: (a) handling the RBC-blood vessel interaction with a fully parallel, highorder boundary integral equation solver; (b) explicit handling of collisions with a parallel constraint-based resolution and detection algorithm. The former is essential for modeling confined flows, while the latter is essential for handling high-volume fraction flows at long time scales without excessively small time steps or fine spatial discretizations.

\section{Our contributions.}

(1) We present a parallel platform for long-time simulations of RBCs through complex blood vessels. The extension to suspensions of various particulates (fibers, rigid bodies etc.) is straightforward from the boundary integral formulation. Flows through several complicated geometries are demonstrated.

(2) We have parallelized a boundary solver for elliptic PDEs on smooth complex geometries in 3D. By leveraging the parallel fast-multipole method of [26] and the parallel forest of quadtrees of [7], we are able to achieve good parallel performance and load balancing.

(3) We have extended the parallel collision handling of [25] to include rigid 3D boundaries composed of patches.

(4) We present weak and strong scalability results of our simulation on the Skylake cluster and weak scaling results on the Knights Landing cluster on Stampede2 at the Texas Advanced Computing Center along with several visualizations of long-time, large-scale blood cell flows through vessels. We observe $49 \%$ strong scaling efficiency for a 32 -fold increase of compute cores. In our largest test on 12288 cores, we simulate 1,048,576 RBCs in a blood vessel composed of $2,097,152$ patches with weak scaling efficiency of $71 \%$ compared to 192 cores (Fig. 5). In each time step, this test uses over three billion degrees of freedom and over four billion surface elements (triangles) for collision.

(5) We are able to simulate realistic human blood flows with RBC volume fractions over $47 \%$ (Fig. 7).

Limitations. Despite the advantages and contributions of the computational framework presented here, our work has some limitations. We have made several simplifications in our model for RBCs. We are restricted to the low Reynolds number regime, i.e., small arteries and capillaries. We use a simplified model for RBCs, assuming the cell membranes to be inextensible and with no inplane shear rigidity. It has been shown that flows in arterioles and capillaries with diameter of $<50 \mu \mathrm{m}$ and RBCs with $5 \mu \mathrm{m}$ diameter have a Reynolds number of $<5 \times 10^{-3}$ [51][9, Section 5.4] with roughly $2 \%$ error in approximating confined flows [1]. This is sufficient for our interest in the qualitative behavior of particulate flows, with the possibility of investigating rheological dynamics in larger channels.

Regarding algorithms, each RBC is discretized with an equal number of points, despite the varied behavior of the velocity through the vessel. Adaptive refinement is required in order to resolve the velocity accurately. Finally, the blood vessel is constructed to satisfy certain geometric constraints that allow for the solution of Eq. (2.5) via singular integration. This can be overcome through uniform refinement, but a parallel adaptive algorithm is required to maintain good performance.

Related work: blood flow. Large-scale simulation of RBC flows typically fall into four categories: (a) Immersed boundary (IB) and immersed interface methods; (b) particle-based methods such as dissipative particle dynamics (DPD) and smoothed particle hydrodynamics $(\mathrm{SPH})$ (c) multiscale network-based approaches and (d) boundary integral equation (BIE) approaches. For a comprehensive review of general blood flow simulation methods, see [12]. IB methods can produce high-quality simulations of heterogeneous particulate flows in complex blood vessels [3, 4, 52]. These methods typically require a finite element solve for each $\mathrm{RBC}$ to compute membrane 
tensions and use IB to couple the stresses with the fluid. This approach quickly becomes costly, especially for high-order elements, and although reasonably large simulation have been achieved [41, 42], large-scale parallelization has remained a challenge. A different approach to simulating blood flow is with multiscale reduced-order models. By making simplifying assumptions about the fluid behavior throughout the domain and transforming the complex fluid system into a simpler flow problem, the macroscopic behaviors of enormous capillary systems can be characterized [33,34] and scaled up to thousands of cores [32]. This comes at a cost of local accuracy; by simulating the flows directly, we are able to accurately resolve local RBC dynamics that are not captured by such schemes.

Particle-based methods have had the greatest degree of success at large-scale blood flow simulations [13, 16, 39, 40]. These types of approaches are extremely flexible in modeling the fluid and immersed particles, but are computationally demanding and usually suffer from numerical stiffness that requires very small time steps for a given target accuracy. For a comprehensive review, see [55]. There have also been recent advances in coupling a particle-based DPD-like scheme with IB in parallel [54,56], but the number of RBCs simulated and the complexity of the boundary seems to be limited.

BIE methods have successfully realized large-scale simulations of millions of RBCs [37] in free space. Recently, new methods for robust handling of collisions between RBCs in high-volume fraction simulations have been introduced [25, 28]. This approach is versatile and efficient due to only requiring discretization of RBCs and blood vessel surfaces, while achieving high-order convergence and optimal complexity implementation due to fast summation methods [21, 38, 43, 44, 47, 48, 59]. To solve elliptic partial differential equations, BIE approaches have been successful in several application domains [6, 49, 50, 57]. However, to our knowledge, there has been no work combining a Stokes boundary solver on arbitrary complex geometries in 3D with a collision detection and resolution scheme to simulate RBC flows at large scale. This work aims to fill this gap, illustrating that this can be achieved in a scalable manner.

Related work: collisions. Parallel collision detection methods are a well-studied area in computer graphics for both shared memory and GPU parallelism [20, 23, 29]. [10, 19] detect collisions between rigid bodies in a distributed memory architecture via domain decomposition. [31] constructs a spatial hash to cull collision candidates and explicitly check candidates that hash to the same value. The parallel geometry and physics-based collision resolution scheme detailed in [53] is most similar to the scheme used in this work. However, such discrete collision detection schemes require small time steps to guarantee detections which can become costly for high-volume fraction simulations.

\section{FORMULATION AND SOLVER OVERVIEW}

\subsection{Problem summary}

We simulate the flow of $N$ cells with deformable boundary surfaces $\gamma_{i}, i=1, \ldots, N$ in a viscous Newtonian fluid in a domain $\Omega \subset$ $\mathbb{R}^{3}$ with a fixed boundary $\Gamma$. The governing partial differential equations (PDEs) describing the conservation of momentum and mass are the incompressible Stokes equations for the velocity $\boldsymbol{u}$ and pressure $p$, combined with velocity boundary conditions on $\Gamma$. Additionally, we model cell membranes as massless, so the velocity
$\mathbf{X}_{t}$ of the points on the cell surface coincides with the flow velocity:

$$
\begin{aligned}
& -\mu \Delta \boldsymbol{u}(\boldsymbol{x})+\nabla p(\boldsymbol{x})=\mathbf{F}(\boldsymbol{x}) \text { and } \nabla \cdot \boldsymbol{u}(\boldsymbol{x})=0, \quad \boldsymbol{x} \in \Omega, \\
& \boldsymbol{u}(\boldsymbol{x})=\boldsymbol{g}(\boldsymbol{x}), \quad \boldsymbol{x} \in \Gamma, \\
& \mathbf{X}_{t}=\boldsymbol{u}(\mathbf{X}), \quad X \in \gamma_{i}(t),
\end{aligned}
$$

where $\mu$ is the viscosity of the ambient fluid; in our simulations, we use a simplified model with the viscosity of the fluid inside the cells also being $\mu$ although our code supports arbitrary viscosity contrast. The right-hand side force in the momentum equation is due to the sum of tension and bending forces $\mathbf{f}=\mathbf{f}_{\sigma}+\mathbf{f}_{b}$; it is concentrated on the cell surfaces. We assume that cell surfaces are inextensible, with bending forces determined by the Canham-Helfrich model [8, 18], based on the surface curvature, and surface tension determined by the surface incompressibility condition $\nabla_{\gamma_{i}} \cdot \boldsymbol{u}=0$ resulting in

$$
\mathbf{F}(\boldsymbol{x})=\sum_{i} \int_{\gamma_{i}} \mathbf{f}(\boldsymbol{y}) \delta(\boldsymbol{x}-\boldsymbol{y}) d \boldsymbol{y}
$$

(see, e.g., [38] for the expressions for $\mathbf{f}$ ). Except on inflow and outflow regions of the vascular network, the boundary condition $\boldsymbol{g}$ is zero, modeling no-slip boundary condition on blood vessel walls.

2.1.1 Boundary integral formulation. To enforce the boundary conditions on $\Gamma$, we use the standard approach of computing $\boldsymbol{u}$ as the sum of the solution $\boldsymbol{u}^{\mathrm{fr}}$ of the free-space equation Eq. (2.1) without boundary conditions but with non-zero right-hand side $\mathbf{F}(\boldsymbol{x})$, and the second term $\boldsymbol{u}^{\Gamma}$ obtained by solving the homogeneous equation with boundary conditions on $\Gamma$ given by $\boldsymbol{g}-\boldsymbol{u}^{\mathrm{fr}}$.

Following the approach of $[25,30,35,36]$, we reformulate Eqs. (2.1) and (2.2) in the integral form. The free-space solution $\boldsymbol{u}^{\mathrm{fr}}$ can be written directly as the sum of the single-layer Stokes potentials $\boldsymbol{u}^{\gamma_{i}}$ :

$$
\boldsymbol{u}^{\gamma_{i}}(\boldsymbol{x})=\left(S_{i} \mathbf{f}\right)(\boldsymbol{x})=\int_{\gamma_{i}} S(\boldsymbol{x}, \boldsymbol{y}) \mathbf{f}(\boldsymbol{y}) d \boldsymbol{y}, \quad \boldsymbol{x} \in \Omega,
$$

where $S(\boldsymbol{x}, \boldsymbol{y})=\frac{1}{8 \pi \mu}\left(\frac{1}{\boldsymbol{r}}+\frac{\boldsymbol{r} \otimes \boldsymbol{r}}{|\boldsymbol{r}|^{3}}\right)$ for viscosity $\mu$ and $\boldsymbol{r}=\boldsymbol{x}-\boldsymbol{y}$.

To obtain $\boldsymbol{u}^{\Gamma}$, we reformulate the homogeneous volumetric PDE with nonzero boundary conditions as a boundary integral equation for an unknown double-layer density $\phi$ defined on the domain boundary $\Gamma$ :

$$
\left(\frac{1}{2} I+D+N\right) \phi=\tilde{D}_{\Gamma} \phi=\boldsymbol{g}-\boldsymbol{u}^{\mathrm{fr}}, \quad \boldsymbol{x} \in \Gamma,
$$

where the double-layer operator is $D \phi(\boldsymbol{x})=\int_{\Gamma} D(\boldsymbol{x}, \boldsymbol{y}) \phi(\boldsymbol{y}) d \boldsymbol{y}$ with double-layer Stokes kernel $D(\boldsymbol{x}, \boldsymbol{y})=\frac{6}{8 \pi}\left(\frac{\boldsymbol{r} \otimes \boldsymbol{r}}{|\boldsymbol{r}|^{5}}(\boldsymbol{r} \cdot \boldsymbol{n})\right.$ for outward normal $\boldsymbol{n}=\boldsymbol{n}(\boldsymbol{y})$. The null-space operator needed to make the equations full-rank is defined as $(N \phi)(\boldsymbol{x})=\int_{\Gamma}(\boldsymbol{n}(\boldsymbol{x}) \cdot \phi(\boldsymbol{y})) \boldsymbol{n}(\boldsymbol{y}) d \boldsymbol{y}$ (cf. [24]). The favorable eigenspectrum of the integral operator in Eq. (2.5) is well-known and allows GMRES to rapidly converge to a solution. One of the key differences between this work and previous free-space large-scale simulations is the need to solve this equation in a scalable way. Once the density $\phi$ is computed, the velocity correction $\boldsymbol{u}^{\Gamma}$ is evaluated directly as $\boldsymbol{u}^{\Gamma}=D \phi$.

The equation for the total velocity $\boldsymbol{u}(\boldsymbol{x})$ at any point $\boldsymbol{x} \in \Omega$ is then given by

$$
\boldsymbol{u}=\boldsymbol{u}^{\mathrm{fr}}+\boldsymbol{u}^{\Gamma}=\sum_{i=1}^{N} \boldsymbol{u}^{\gamma_{i}}+\boldsymbol{u}^{\Gamma}
$$


In particular, this determines the update equation for the boundary points of cells; see Eq. (2.3).

Contact formulation. In theory, the contacts between surfaces are prevented by the increasing fluid forces as surfaces approach each other closely. However, ensuring accuracy of resolving forces may require prohibitively fine sampling of surfaces and very small time steps, making large-scale simulations in space and time impractical. At the same time, as shown in [24], interpenetration of surfaces results in a catastrophic loss of accuracy due to singularities in the integrals.

To guarantee that our discretized cells remain interference-free, we augment Eqs. (2.1) and (2.2) with an explicit inequality constraint preventing collisions. We define a vector function $V(t)$ with components becoming strictly negative if any cell surfaces intersect each other, or intersect with the vessel boundaries $\Gamma$. More specifically, we use the space-time interference volumes introduced in [17] and applied to 3D cell flows in [25]. Each component of $V$ corresponds to a single connected overlap. The interference-free constraint at time $t$ is then simply $V(t) \geq 0$.

For this constraint to be satisfied, the forces $\mathbf{f}$ are augmented by an artificial collision force, i.e., $\mathbf{f}=\mathbf{f}_{b}+\mathbf{f}_{\sigma}+\mathbf{f}_{c}, \mathbf{f}_{c}=\nabla_{u} V^{T} \lambda$, where $\lambda$ is the vector of Lagrange multipliers, which is determined by the additional complementarity conditions:

$$
\lambda(t) \geq 0, \quad V(t) \geq 0, \quad \lambda(t) \cdot V(t)=0,
$$

at time $t$, where all inequalities are to be understood componentwise.

To summarize, the system that we solve at every time step can be formulated as follows, where we separate equations for different cells and global and local parts of the right-hand side, as it is important for our time discretization:

$$
\begin{aligned}
& X_{t}=\left(\sum_{j \neq i} S_{j} \mathbf{f}_{j}+D \phi\right)+S_{i} \mathbf{f}_{i}, \quad \text { for points on } \gamma_{i}, \\
& \nabla_{\gamma_{i}} \cdot \boldsymbol{X}_{t}=0, \quad \mathbf{f}_{j}=\mathbf{f}\left(\boldsymbol{X}_{j}, \sigma_{j}, \lambda\right), \\
& B_{\Gamma} \phi=\boldsymbol{g}-\sum_{j} S_{j} \mathbf{f}_{j}, \quad \text { for points on } \Gamma, \\
& \lambda(t) \geq 0, \quad V(t) \geq 0, \quad \lambda(t) \cdot V(t)=0 .
\end{aligned}
$$

At every time step, (2.11) results in coupling of all close $\gamma_{i}$ 's, which requires a non-local computation. We follow the approach detailed in $[24,25]$ to define and solve the nonlinear complementarity problem ( $\mathrm{NCP})$ arising from cell-cell interactions in parallel, and extend it to prevent intersection of cells with the domain boundary $\Gamma$, as detailed in Section 4.

\subsection{Algorithm Overview}

Next, we summarize the algorithmic steps used to solve the constrained integral equations needed to compute cell surface positions and fluid velocities at each time step. In the subsequent sections, we detail the parallel algorithms we developed to obtain good weak and strong scalability, as shown in Section 5.

Overall Discretization. RBC surfaces are discretized using a spherical harmonic representation, with surfaces sampled uniformly in the standard latitude-longitude sphere parametrization. The blood vessel surfaces $\Gamma$ are discretized using a collection of high-order tensor-product polynomial patches, each sampled at Clenshaw-Curtis quadrature points. The space-time interference volume function $V(t)$ is computed using a piecewise-linear approximation as described in [25]. For time discretization, we use a locallyimplicit first order time-stepping (higher-order time stepping can be easily incorporated). Interactions between RBCs and the blood vessel surfaces are computed explicitly, while the self-interaction of a single RBC is computed implicitly.

The state of the system at every time step is given by a triple of distributed vectors $(X, \sigma, \lambda)$. The first two (cell surface positions and tensions) are defined at the discretization points of cells. The vector $\lambda$ has variable length and corresponds to connected components of collision volumes. We use the subscript $i$ to denote the subvectors corresponding to $i$-the cell. $X$ and $\sigma$ are solved as a single system that includes the incompressibility constraint Eq. (2.9). To simplify exposition, we omit $\sigma$ in our algorithm summary, which corresponds to dropping $\mathbf{f}_{\sigma}$ in the Stokes equation, and dropping the surface incompressibility constraint equation.

Algorithm summary. At each step $t$, we compute the new positions $X_{i}^{+}$and collision Lagrange multipliers $\lambda^{+}$at time $t^{+}=$ $t+\Delta t$. We assume that in the initial configuration there are no collisions, so the Lagrange multiplier vector $\lambda$ is zero. Discretizing in time, Eq. (2.8) becomes

$$
X_{i}^{+}=X_{i}+\Delta t\left(\sum_{j \neq i} S_{j} \mathbf{f}_{j}\left(X_{j}, \lambda\right)+D \phi\left(X_{j}, \lambda\right)\right)+\Delta t S_{i} \mathbf{f}_{i}\left(X_{i}^{+}, \lambda^{+}\right) .
$$

At each single time step, we perform the following steps to obtain $\left(X^{+}, \lambda^{+}\right)$from $(X, \lambda)$. Below evaluation of integrals implies using appropriate (smooth, near-singular or singular) quadrature rules on cell or blood vessel surfaces.

(1) Compute the explicit part $\boldsymbol{b}$ of the position update (first term in Eq. (2.8)).

(a) Evaluate $\boldsymbol{u}^{\text {fr }}$ from $(\boldsymbol{X}, \lambda)$ on $\Gamma$ with Eq. (2.4).

(b) Solve Eq. (2.5) for the unknown density $\phi$ on $\Gamma$ using GMRES.

(c) For each cell, evaluate $\boldsymbol{u}_{i}^{\Gamma}=D \phi$ at all cell points $\boldsymbol{X}_{i}$.

(d) For each cell $i$, compute the contributions of other cells to $\boldsymbol{X}_{i}^{+}: \boldsymbol{b}_{i}^{c}=\boldsymbol{u}^{\mathrm{fr}}-u^{\gamma_{i}}=\sum_{j \neq i} S_{j} \mathbf{f}_{j}$.

(e) Set $\boldsymbol{b}_{i}=\boldsymbol{u}_{i}^{\Gamma}+\boldsymbol{b}_{i}^{c}$.

(2) Perform the implicit part of the update: solve the NCP obtained by treating the second (self-interaction) term in Eq. (2.8) while enforcing the complementarity constraints Eq. (2.7), i.e., solve

$$
\begin{array}{r}
X_{i}^{+}=X_{i}+\Delta t\left(\boldsymbol{b}_{i}+S_{i} f_{i}\left(\boldsymbol{X}_{i}^{+}, \lambda^{+}\right)\right), \\
\lambda\left(t^{+}\right) \geq 0, \quad V\left(t^{+}\right) \geq 0, \quad \lambda\left(t^{+}\right) \cdot V\left(t^{+}\right)=0 .
\end{array}
$$

Items $1 \mathrm{a}$ to $1 \mathrm{~d}$ all require evaluation of global integrals, evaluated as sums over quadrature points; we compute these sums in parallel with PVFMM. In particular, Item $1 \mathrm{~b}$ uses PVFMM as a part of each matrix-vector product in the GMRES iteration. These matrix-vector product, as well as Items $1 \mathrm{a}, 1 \mathrm{c}$ and $1 \mathrm{~d}$ require near-singular integration to compute the velocity accurately near RBC and blood vessel surfaces; this requires parallel communication to find non-local evaluation points. Details of these computations are discussed in Section 3. 
The NCP is solved using a sequence of linear complementarity problems (LCPs). Algorithmically, this requires parallel searches of collision candidate pairs and the repeated application of the distributed LCP matrix to distributed vectors. Details of these computations are provided in Section 4.

Other parallel quadrature methods. Various other parallel algorithms are leveraged to perform boundary integrals for the vessel geometry and RBCs. To compute $\boldsymbol{u}^{\gamma_{i}}(\boldsymbol{X})$ for $\boldsymbol{X} \in \gamma_{i}$, the schemes presented in [48] are used to achieve spectral convergence for single-layer potentials by performing a spherical harmonic rotation and apply the quadrature rule of [14]. We use the improved algorithm in [28] to precompute the singular integration operator and substantially improve overall complexity. To compute $\boldsymbol{u}^{\gamma_{i}}(\boldsymbol{X})$ for $X$ close to, but not on $\gamma_{i}$, we follow the approaches of [28, 43], which use a variation of the high-order near-singular evaluation scheme of [58]. Rather than extrapolating the velocity from nearby check points as in Section 3, we use [48] to compute the velocity on surface, upsampled quadrature on $\gamma_{i}$ to compute the velocity at check points and interpolate the velocity between them to the desired location. We mention these schemes for the sake of completeness; they are not the primary contribution of this work, but are critical components of the overall simulation.

\section{BOUNDARY SOLVER}

The main challenge in incorporating prescribed flow boundary conditions $\boldsymbol{g}$ on the domain boundary $\Gamma$ is the approximation and solution of the boundary integral problem Eq. (2.5). Upon spatial discretization, this is an extremely large, dense linear system that must be solved at every time step due to the changing free space solution $\boldsymbol{u}^{\mathrm{fr}}$ on the right hand side. Since we aim at a scalable implementation, we do not assemble the operator on the left hand side but only implement the corresponding matrix-vector multiplication, i.e., its application to vectors. Combined with an iterative solver such as GMRES, this matrix-vector multiply is sufficient to solve Eq. (2.5). Application of the double-layer operator $D$ to vectors amounts to a near-singular quadrature for points close to $\Gamma$. Controlling the error in this computation requires a tailored quadrature scheme. This scheme is detailed below, where we put a particular emphasis on the challenges due to our parallel implementation.

\subsection{Quadrature for integral equation}

The domain boundary $\Gamma$ is given by a collection of non-overlapping patches $\Gamma=\bigcup_{i} P_{i}(Q)$, where $P_{i}: Q \rightarrow \mathbb{R}^{3}$ is defined on $Q=$ $[-1,1]^{2}$. We use the Nyström discretization for Eq. (2.5). Since $D(\boldsymbol{x}, \boldsymbol{y})$ is singular, this requires a singular quadrature scheme for the integral on the right-hand side. We proceed in several steps, starting with the direct non-singular discretization, followed by a distinct discretization for the singular and near-singular case.

Non-singular integral quadrature. We discretize the integral in Eq. (2.5), for $\boldsymbol{x} \notin \Gamma$, by rewriting it as an integral over a set of patches and then apply a tensor-product $q$ th order Clenshaw-Curtis rule to each patch:

$$
\boldsymbol{u}(\boldsymbol{x})=\sum_{i} \int_{P_{i}} D(\boldsymbol{x}, \boldsymbol{y}) \phi(\boldsymbol{y}) d \boldsymbol{y}_{P_{i}} \approx \sum_{i} \sum_{j=0}^{q^{2}} D\left(\boldsymbol{x}, \boldsymbol{y}_{i j}\right) w_{i j} \phi\left(\boldsymbol{y}_{i j}\right)
$$

where $\boldsymbol{y}_{i j}=P_{i}\left(\boldsymbol{t}_{j}\right)$ and $\boldsymbol{t}_{j} \in[-1,1]^{2}$ is the $j$ th quadrature point and $w_{i j}$ is the corresponding quadrature weight. We refer to the points $\boldsymbol{y}_{i j}$ as the coarse discretization of $\Gamma$ and introduce a single global index $\boldsymbol{y}_{\ell}=\boldsymbol{y}_{i j}$ with $\ell=\ell(i, j)=(i-1) q^{2}+j, \ell=1, \ldots, N$, where $N$ is the total number of quadrature nodes. We can then rewrite the right-hand side of (3.1) compactly as the vector dot product $W(\boldsymbol{x}) \cdot \phi$, where $\phi_{\ell}=\phi\left(\boldsymbol{y}_{\ell}\right)$ and $W_{\ell}(\boldsymbol{x})=D\left(\boldsymbol{x}, \boldsymbol{y}_{\ell}\right) w_{\ell}$ are the quadrature weights in Eq. (3.1).

As $x \rightarrow \Gamma$ for $x \in \Omega$, the integrand becomes more singular and the accuracy of this quadrature rapidly decreases due to the singularity in the kernel $D$. This requires us to construct a singular integral discretization for $\boldsymbol{x}=\boldsymbol{y}_{\ell}, \ell=1, \ldots, N$, and general points on $\Gamma$, which is discussed next. Note that the same method is used for evaluation of the velocity values at points close to the surface, once the equation is solved (near-singular integration).

Singular and near-singular integral quadrature. We take

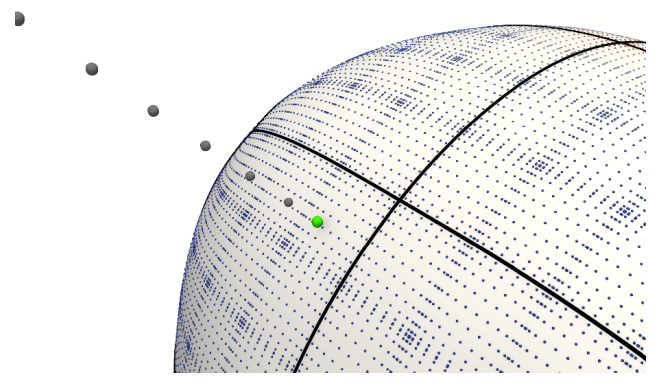

Figure 2: Schematic of our unified singular/near-singular quadrature scheme. A boundary $\Gamma$ is shown along with a set of patches (patch edges shown in black). We evaluate the velocity due to $\Gamma$ at the check points (gray dots off-surface) using the fine discretization (small dots on-surface) and extrapolate these values to the target point (green). The target point may be on or near $\Gamma$. The fine discretization subdivides the patches in the coarse discretization into 16 patches, each with an 11th-order tensor-product Clenshaw-Curtis quadrature rule.

an approach similar to [22]. The idea is to evaluate the integral sufficiently far from the surface using the non-singular quadrature rule (3.1) on an upsampled mesh, and then to extrapolate the accurate values towards the surface. Concretely, to compute the singular integral at a point $\boldsymbol{x}$ near or on $\Gamma$, we use the following steps:

(1) Upsample $\phi$ using $q$ th order interpolation, i.e., $\phi^{\text {up }}=U \phi$, where $\phi^{\text {up }}$ is the vector of $N k$ samples of the density and $U$ is the interpolation operator. To be precise, we subdivide each patch $P_{i}$ into $k$ square subdomains $P_{i k}$ and use ClenshawCurtis nodes in each subdomain. We subdivide uniformly, i.e., $P_{i}$ is split into $k=4^{\eta}$ patches for an integer $\eta$. This is the fine discretization of $\Gamma$. We use $W^{\text {up }}$ to denote the weights for Eq. (3.1) the fine discretization quadrature points.

(2) Find the closest point $\boldsymbol{y}=P\left(u^{*}, v^{*}\right)$ to $\boldsymbol{x}$ on $\Gamma$ for some patch $P$ on $\Gamma$ with $u^{*}, v^{*} \in[-1,1](\boldsymbol{y}=\boldsymbol{x}$ if $\boldsymbol{x} \in \Gamma)$.

(3) Construct check points $c_{q}=c_{q}(\boldsymbol{x})=\boldsymbol{y}-(R+i r) \boldsymbol{n}\left(u^{*}, v^{*}\right)$, $i=0, \ldots, p$, where $\boldsymbol{n}(u, v)$ is the outward normal vector to $\Gamma$ at $P(u, v)$.

(4) Evaluate the velocity at the check points:

$$
\boldsymbol{u}\left(c_{q}(\boldsymbol{x})\right) \approx W^{\mathrm{up}}\left(c_{q}\right) \cdot \phi^{\text {up }}, \quad i=0, \ldots, p .
$$


(5) Extrapolate the velocity from the check points to $x$ with 1D polynomial extrapolation:

$$
\begin{gathered}
\left.\boldsymbol{u}(\boldsymbol{x}) \approx \sum_{q} e_{q} \boldsymbol{u}\left(c_{q}(\boldsymbol{x})\right)=\left(\sum_{q} e_{q} W^{\mathrm{up}}\left(c_{q}\right)\right)\right) U \phi \\
=W^{\mathrm{s}}(\boldsymbol{x}) \cdot \phi
\end{gathered}
$$

where $e_{q}$ are the extrapolation weights.

In this work, the parameters $R, p, r$ and $\eta$ are chosen empirically to balance the error in the accuracy of $W^{\mathrm{up}}\left(c_{q}\right) \cdot \phi^{\mathrm{up}}$ and the extrapolation to $\boldsymbol{x}$. A schematic of this quadrature procedure is shown in Fig. 2.

Discretizing the integral equation. With the singular integration method described above, we take $\boldsymbol{x}=\boldsymbol{y}_{\ell}, \ell=1 \ldots N$, and obtain the following discretization of Eq. (2.5):

$$
\left(\frac{1}{2} I+A\right) \phi=\boldsymbol{g}, \quad A_{\ell m}=W_{m}^{\mathrm{s}}\left(\boldsymbol{y}_{\ell}\right)+N_{i j},
$$

where $\boldsymbol{g}$ is the boundary condition evaluated at $\boldsymbol{y}_{\ell}, W_{m}^{s}(\boldsymbol{x})$ is the $m$ th component of $W^{s}(\boldsymbol{x})$ and $N_{i j}$ is the appropriate element of the rank-completing operator in Eq. (2.5).

The dense operator $A$ is never assembled explicitly. We use GMRES to solve Eq. (3.5), which only requires application of $A$ to vectors $\phi$. This matrix-vector product is computed using the steps summarized above.

Extrapolation and upsampling are local computations that are parallelized trivially if all degrees of freedom for each patch are on a single processor. The main challenges in parallelization of the above singular evaluation are 1) initially distributing the patches among processors, 2) computing the closest point on $\Gamma$ and 3) evaluating the velocity at the check points. The parallelization of these computations is detailed in the remainder of this section.

Far evaluation. To compute the fluid velocity away from $\Gamma$, where Eq. (2.5) is non-singular, i.e., at the check points, the integral can be directly evaluated using Eq. (3.1). Observing that Eq. (3.1) has the form of an $\mathrm{N}$-body summation, we use the fast-multipole method [15] to evaluate it for all target points at once. We use the parallel, kernel-independent implementation Parallel Volume Fast Multipole Method (PVFMM) [26, 27], which has been demonstrated to scale to hundreds of thousands of cores. PVFMM handles all of the parallel communication required to aggregate and distribute the contribution of non-local patches in $O(N)$ time.

\subsection{Distributing geometry and evaluation parallelization}

We load pieces of the blood vessel geometry, which is provided as a quad mesh, separately on different processors. Each face of the mesh has a corresponding polynomial $P_{i}$ defining the $i$ th patch.

The $k$ levels of patch subdivision induce a uniform quadtree structure within each quad. We use the p4est library [7] to manage this surface mesh hierarchy, keep track of neighbor information, distribute patch data and to refine and coarsen the discretization in parallel. The parallel quadtree algorithms provided by p4est are used to distribute the geometry without replicating the complete surface and polynomial patches across all processors. p4est also determines parent-child patch relationships between the coarse and fine discretizations and the coordinates of the child patches to which we interpolate.

Once the geometry is distributed, constructing check points, all necessary information for upsampling and extrapolation are either available on each processor or communicated by PVFMM. This allows these operations to be embarassingly parallel.

\subsection{Parallel closest point search}

To evaluate the solution at a point $x$, we must find the closest point $\boldsymbol{y}$ on the boundary to $\boldsymbol{x}$. The distance $\|\boldsymbol{x}-\boldsymbol{y}\|_{2}$ determines whether or not near-singular integration is required to compute the velocity at $\boldsymbol{x}$. If it is, $\boldsymbol{y}$ is used to construct check points.

In the context of this work, the point $x$ is on the surface of an RBC, which may be on a different processor than the patch containing $\boldsymbol{y}$. This necessitates a parallel algorithm to search for $\boldsymbol{y}$. For that purpose, we extend the spatial sorting algorithm from [25, Algorithm 1] to support our fixed patch-based boundary and detect near pairs of target points and patches.

a. Construct a bounding box $B_{P, \epsilon}$ for the near-zone of each patch. We choose a distance $d_{\epsilon}$ so that for all points $z$ further away than $d_{\epsilon}$ from $P$, the quadrature error of integration over $P$ is bounded by $\epsilon$. The set of points closer to $P$ than $d_{\epsilon}$ is the near-zone of $P$. We inflate the bounding box $B_{P}$ of $P$ by $d_{\epsilon}$ along the diagonal to obtain $B_{P, \epsilon}$ to contain all such points.

b. Sample $B_{P, \epsilon}$ and compute a spatial hash of the samples and $\boldsymbol{x}$. Let $H$ be the average diagonal length of all $B_{P, \epsilon}$. We sample the volume contained in $B_{P, \epsilon}$ with equispaced samples of spacing $h_{P}<H$. Using a spatial hash function, (such as Morton ordering with a spatial grid of spacing $H$ ), we assign hash values to bounding box samples and $\boldsymbol{x}$ to be used as a sorting key. This results in a set of hash values that define the near-zone of $\Gamma$.

c. Sort all samples by the sorting key. Use the parallel sort of [45] on the sorting key of bounding box samples and that of $\boldsymbol{x}$. This collects all points with identical sorting key (i.e., close positions) and places them on the same processor. If the hash of $\boldsymbol{x}$ matches the hash of a bounding box sample, then $x$ could require nearsingular integration, which we check explicitly. Otherwise, we can assume $\boldsymbol{x}$ is sufficiently far from $P$ and does not require singular integration.

d. Compute distances $\left\|\boldsymbol{x}-P_{i}\right\|$. For each patch $P_{i}$ with a bounding box key of $\boldsymbol{x}$, we locally solve the minimization problem $\min _{(u, v) \in[-1,1]^{2}}\left\|x-P_{i}(u, v)\right\|$ via Newton's method with a backtracking line search. This is a local computation since $\boldsymbol{x}$ and $P_{i}$ were communicated during the Morton ID sort.

e. Choose the closest patch $P_{i}$. We perform a global reduce on the distances $\left\|\boldsymbol{x}-P_{i}\right\|$ to determine the closest $P_{i}$ to $\boldsymbol{x}$ and communicate back all the relevant information required for singular evaluation back to $\boldsymbol{x}$ 's processor.

\section{PARALLEL COLLISION HANDLING}

We prevent collisions of RBCs with other RBCs and with the vessel surface $\Gamma$ by solving the NCP given in Eqs. (4.1) and (4.2). This is a nonsmooth and non-local problem, whose assembly and efficient solution is particularly challenging in parallel.In this section, we summarize our constraint-based approach and algorithm. 
We have integrated piecewise polynomial patches into the framework of [25] for parallel collision handling, to which we refer the reader for a more detailed discussion. The key step to algorithmically unify RBCs and patches is to form a linear triangle mesh approximation of both objects. We now want to enforce that these meshes are collision-free subject to the physics constraints in Eq. (4.1).

We linearize the NCP and solve a sequence of LCPs whose solutions converge to the NCP solution. At a high-level, the collision algorithm proceeds as follows:

(1) Find triangle-vertex pairs of distinct meshes that are candidates for collision.

(2) Compute $V\left(t^{+}\right)=V\left(t^{+, 0}\right)$. If any triangle-vertex pairs on distinct meshes collide, the corresponding component of $V(t)$ will be negative.

(3) While $V_{i}\left(t^{+, k}\right)<0$ for any $i$ :

(a) Suppose $m$ components of $V(t)$ are negative

(b) Solve the following linearized version of Eqs. (4.1) and (4.2)

$$
\begin{gathered}
X_{i}^{+, k}=X_{i}+\Delta t\left(\boldsymbol{b}_{i}+S_{i}\left(\mathbf{f}_{i}\left(X_{i}^{+, k}, \lambda^{+, k}\right)\right),\right. \\
\lambda\left(t^{+}\right) \geq 0, \quad L\left(t^{+, k}\right) \geq 0, \quad \lambda\left(t^{+, k}\right) \cdot L\left(t^{+, k}\right)=0, \\
\text { where } \quad L(t)=V(t)+\nabla_{u} V^{T} \Delta \boldsymbol{X}_{i}(t)
\end{gathered}
$$

for the $k$ th iteration of the loop and $\boldsymbol{X}_{i}^{+, k}=\boldsymbol{X}_{i}+\Delta \boldsymbol{X}_{i}\left(t^{+, k}\right)$. (c) Find new candidate triangle-vertex pairs and compute $V\left(t^{+, k}\right)$.

Here, $t^{+, k}$ is the intermediate time step at which a new candidate position $X_{i}^{+, k}$ occurs. This approach of iteratively solving an NCP with sequence of LCPs was shown to converge superlinearly in [11] In [53], the authors demonstrate that one LCP linearization can approximate the NCP accurately; our algorithm uses around seven LCP solves to approximately solve the NCP. Upon convergence of this algorithm, we are guaranteed that our system is collision-free.

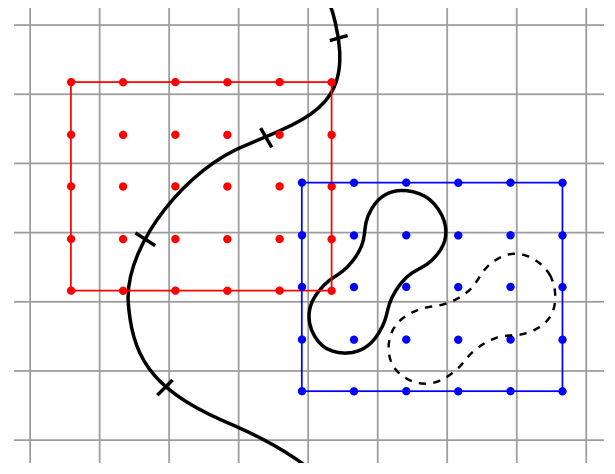

Figure 3: A 2D depiction of the parallel candidate collision pair algorithm. Shown is the implicit spatial grid (gray), a piece of the blood vessel $\Gamma$ (open black curve), an $R B C \gamma_{i}$ at the current time step (closed black curve) and at the next time step (dotted closed back curve). Also shown is the space-time bounding box and bounding box samples of a single patch (red square and red dots) and an RBC (blue square and blue dots).

To solve the LCP in Item $3 \mathrm{~b}$, we follow the approach detailed in [24, Section 3.2.2, Section 3.3]. We reformulate the problem first in standard LCP form with diagonally-dominant system matrix $B$, then solve an equivalent root finding problem by applying a minimummap Newton's method. This can be restructured to use GMRES, so we only need to repeatedly apply $B$ to vectors to solve the LCP. Each entry $\boldsymbol{B}_{i j}$ is the change in the $j$ th contact volume induced by the $k$ th contact force, which is explicitly defined in [25, Algorithm $3]$. This means that $\boldsymbol{B}$ is of size $m \times m$, where $m$ is the number of collisions, but is extremely sparse. We need not store the entire matrix explicitly; we only compute the non-zero entries and store them in a distributed hash-map. Computing these matrix elements requires an accumulation of all coupled collision contributions to the velocity, which requires just a sparse MPI_All_to_Allv to send each local contribution to the process containing $V_{i}\left(t^{+, k}\right)$.

An important step to ensure good scaling of our collision handling algorithm is to minimize the number of triangle-vertex pairs that are found in Item 1. One could explicitly compute an all-to-all collision detection on all meshes in the system, but this requires $O\left(N^{2}\right)$ work and global communication. We perform a high-level filtering first to find local candidate collision mesh pairs, then only communicate and compute the required $O(m)$ information. Since spatially-near mesh pairs may be on different processors, we need a parallel algorithm to compute these collision candidates.

To address this, we reuse Items a to c from Section 3.3 and adapt it to this problem. For each mesh in the system, we form the spacetime bounding box of the mesh: the smallest axis-aligned bounding box containing the mesh at positions $X_{i}$ and $X_{i}^{+}$, as shown in Fig. 3. For patches $P_{i}$, note that $P_{i}^{+}=P_{i}$. This means one can reuse the bounding box of $P_{i}$ constructed in Section 3.3 for this purpose and simply set $d_{\epsilon}$ to zero. After forming all space-time bounding boxes for the meshes of all patches and RBCs, we apply steps Items b and $\mathrm{c}$ directly to these boxes. Item $\mathrm{c}$ will communicate meshes with the same spatial sorting key to the same processor; these meshes are collision candidate pairs. Once the computation is local and candidate collision pairs are identified, we can proceed with the NCP solution algorithm described above.

\section{RESULTS}

In this section, we present scalability results for our blood flow simulation framework on various test geometries, simulations with various volume fractions and demonstrate the convergence behavior of our numerical methods.

\subsection{Implementation and example setup}

Architecture and software libraries. We use the Stampede2 system at the Texas Advanced Computing Center (TACC) to study the scalability of our algorithms and implementation. Stampede2 has two types of compute nodes, the Knights Landing (KNL) compute nodes and the Skylake (SKX) compute nodes. The SKX cluster has 1,736 dual-socket compute nodes, each with two 24 -core $2.1 \mathrm{GHz}$ CPUs and 192GB of memory. The KNL cluster has 4,200 compute nodes, with a 68-core Intel Xeon Phi 7250 1.4Ghz CPUs and 96GB of memory plus 16GB of high-speed MCDRAM. We run our simulations in a hybrid distributed-shared memory fashion: we run one MPI process per node, with one OpenMP thread per hardware core. Our largest simulations use 256 SKX and 512 KNL nodes.

We leverage several high-performance libraries in our implementation. We use PETSc's [2] parallel matrix and vector operations, and 


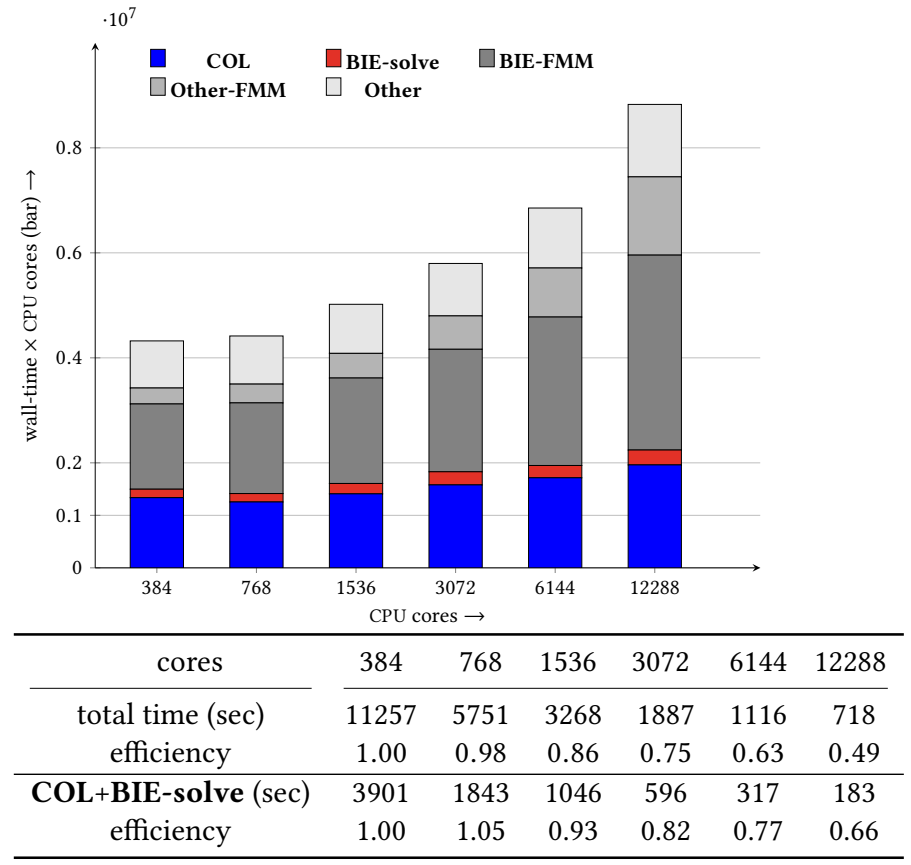

Figure 4: Strong scalability of a simultion with 40960 RBCs on Stampede's SKX partition for the vessel network geometry shown in Fig. 1. The vessel is discretized with 40960 polynomial patches. Shown in the bar graph is a breakdown of the compute resources (wall-time $\times C P U$ cores) required by the individual components for a simulation with 10 time steps on 384 to 12288 cores. The compute resources used by the main algorithms presented in this paper are $\mathbf{C O L}$ (collision handling), BIEsolve (computation of $\boldsymbol{u}^{\Gamma}$, not including FMM calls). Shown in different gray scales are the compute resources required by FMM (BIE-FMM and Other-FMM) and other operations (Other). Shown in the table are the compute time and the parallel efficiency for the overall computation and for the sum of $\mathbf{C O L}$ and BIE-solve. For the collision avoidance and the boundary solve we observe a parallel efficiency of $66 \%$ for a 32-fold increase from 384 to 12288 CPU cores.

its parallel GMRES solver. Management and distribution of patches describing the blood vessel geometry uses the p4est library [7], and we use PVFMM [26] for parallel FMM evaluation. We also heavily leverage Intel MKL for fast dense linear algebra routines at the core of our algorithms and paraview for our visualizations.

Discretization and example setup. For all test cases we present, we discretize each RBC with 544 quadrature points and 2,112 points for collision detection. The blood vessel geometry is represented with 8th order tensor-product polynomial patches with 121 quadrature points per patch and 484 equispaced points for collision detection. The parameters chosen for singular/near-singular integration are $p=8$ and $\eta=1$, with $R=r=.15 L$ for strong scaling tests and $R=r=.1 L$ for weak scaling tests. The value of $L$ is the square root of the surface area of the patch containing the closest point to the target, called the patch size; this choice allows for a consistent extrapolation error over the entirety of $\Gamma$.

Since our scaling tests are performed on complex, realistic blood vessel geometries, we must algorithmically generate our initial simulation configuration. We prescribe portions of the blood vessel

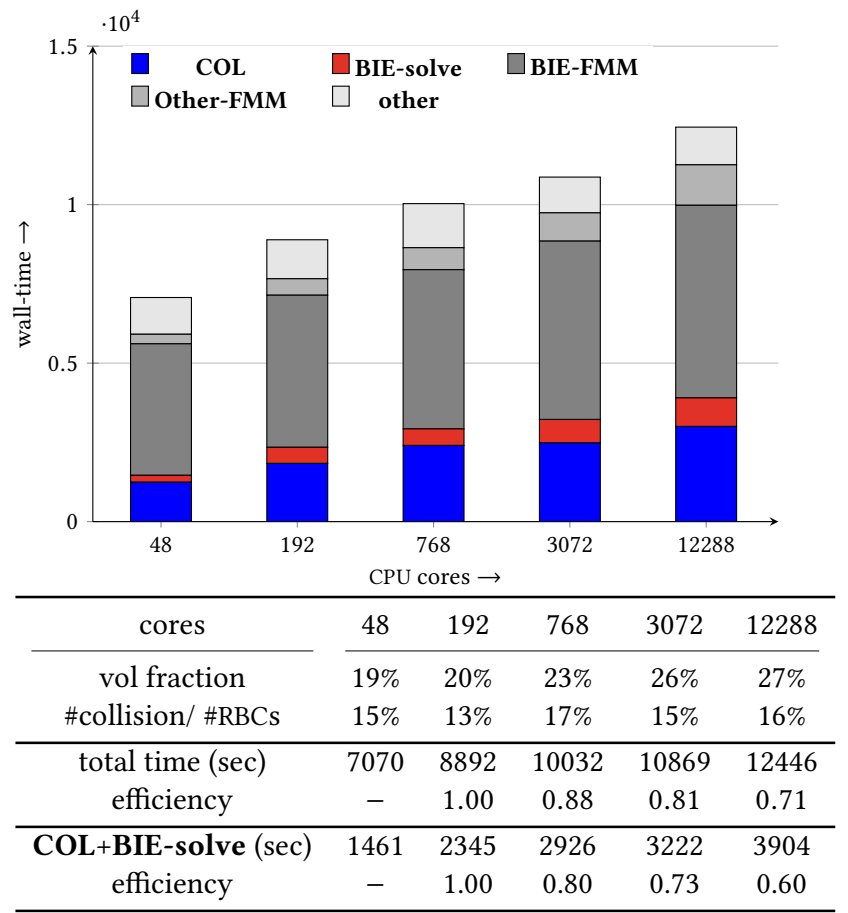

Figure 5: Weak scalability on Stampede's SKX partition with node grain size of 4096 RBCs and 8192 polynomial patches per compute node (each node has 48 cores) for the vessel geometry shown in Fig. 8. Increasing the number of RBCs and boundary patches is realized by decreasing the size of the RBCs as discussed in Section 5.2. Shown in the bar graph is a breakdown of wall-time spent in individual components for a simulation with 10 time steps on 136 to 12288 cores (i.e., 4 to 256 nodes). The explanation of the labels used in the legend is detailed in Fig. 4. Additionally, we show the volume fraction of RBCs for each simulation, as well as the percentage of vesicles where the RBC-RBC or $R B C$-vessel collision prevention is active. We report the parallel scalability with respect to 192 cores, as the smallest simulation is in a single node and no MPI communication is necessary. The largest simulation has 1,048,576 RBCs and 2,097,152 polynomial patches and an overall number of 3,042,967,552 unknowns per time step.

as inflow and outflow regions and appropriately prescribe positive and negative parabolic flows (inlet and outlet flow) as boundary conditions, such that the total fluid flux is zero. To populate the blood vessel with RBCs, we uniformly sample the volume of the bounding box of the vessel with a spacing $h$ to find point locations inside the domain at which we place RBCs in a random orientation. We then slowly increase the size of each RBC until it collides with the vessel boundary or another RBC; this determines a single RBC's size. We continue this process until all RBCs stop expanding; this means that we are running a simulation of RBCs of various sizes. We refer to this process as filling the blood vessel with RBCs. This typically produces RBCs of radius $r$ with $r_{0}<r<2 r_{0}$ with $r_{0}$ chosen proportional to $h$. This is a precomputation for our simulation, so we do not include this step in the timings we report for weak and strong scaling. We emphasize that these simulations are primarily for scaling purposes of our algorithms and are not expected to 


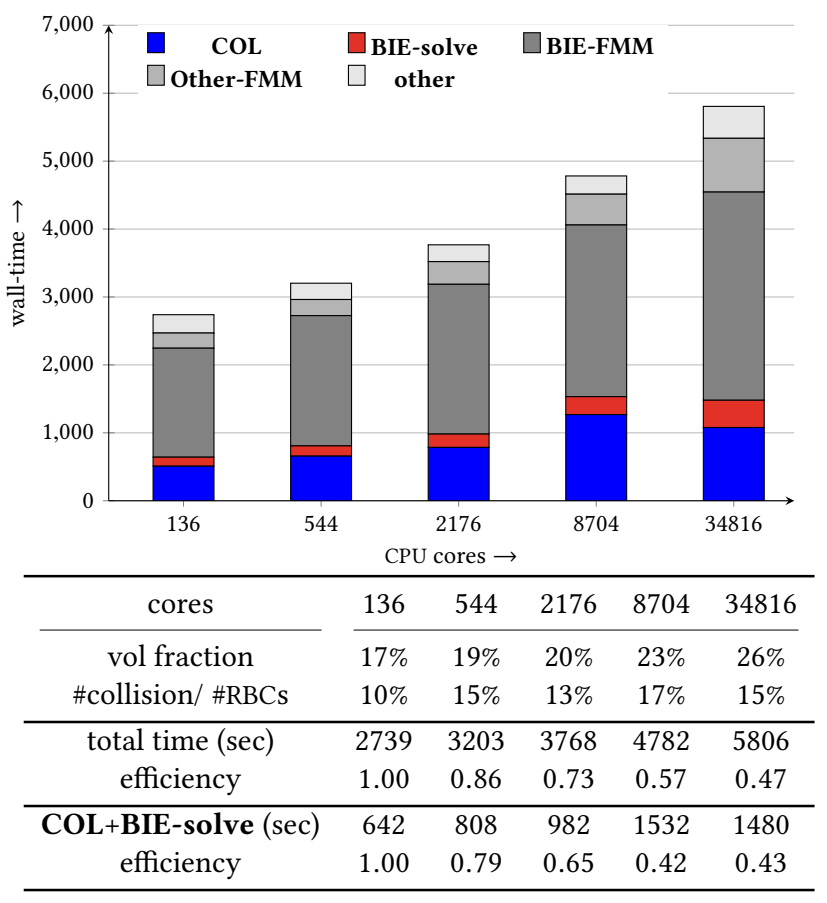

Figure 6: Same as Fig. 5 but on Stampede2's KNL partition with 512 RBCs and 1024 vessel boundary patches per node (each node has 68 cores). We find an overall parallel scalability of $47 \%$ for a 256-fold increase of the problem size.

represent true blood flows. The platform can of course be applied to length scales where viscous flow is a valid assumption.

Additionally, RBCs in such a confined flow will collide with the blood vessel wall if special care is not taken near the outflow part of the boundary. We define regions near the inlet and outlet flows where we can safely add and remove RBCs. When an RBC $\gamma_{i}$ is within the outlet region, we subtract off the velocity due to $\gamma_{i}$ from the entire system and move $\gamma_{i}$ into an inlet region such that the arising $\mathrm{RBC}$ configuration is collision-free.

Limiting GMRES iterations. We have observed that the GMRES solver typically requires 30 iterations or less for convergence for almost all time steps, but the number of needed iterations may vary more in the first steps. To simulate the amount of work in a typical simulation time step, we cap the number of GMRES iterations at 30 and report weak and strong scaling for these iterations. A more detailed analysis of this behavior is needed.

\subsection{Parallel scalability}

Here, we present strong and weak scalability results for our RBC simulations. We decompose the time required for a complete simulation into the following categories:

- COL: detection and resolution of collisions among RBCs and between RBCs and the vessel walls;

- BIE-solve: computing $\boldsymbol{u}^{\Gamma}$, not including FMM calls. This includes all of the steps for singular/near-singular integration in Section 3 except the evaluation $\boldsymbol{u}^{\Gamma}$ at the check points.

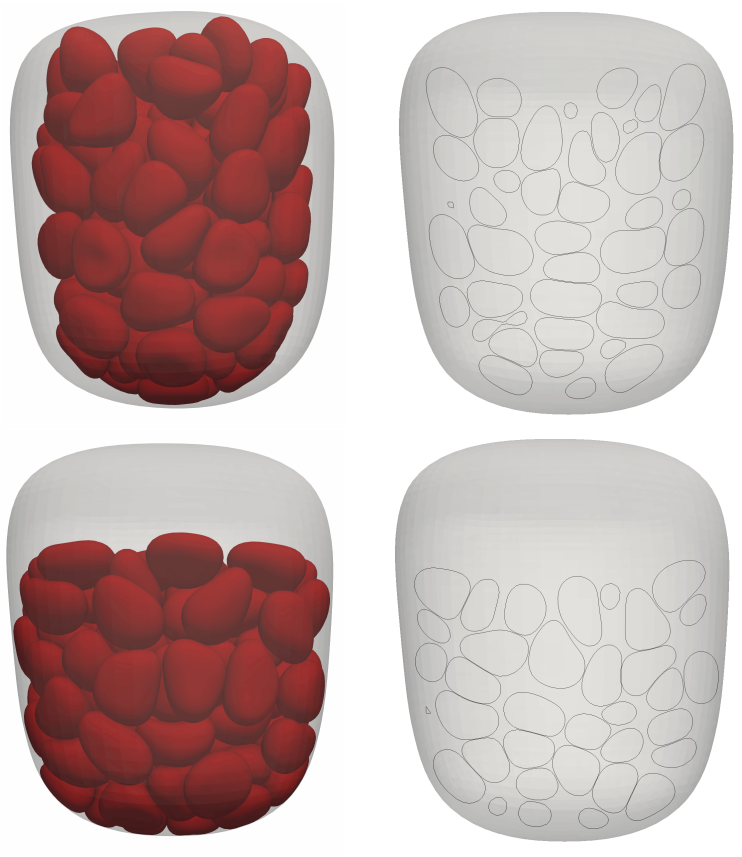

Figure 7: Shown is a high-volume fraction sedimentation due to gravitational force. The initial configuration (top figures) has a volume fraction of $47 \%$. As the cells sediment to the lower part of the domain (bottom figures), the local volume fraction of the final state in this lower part of the domain is around 55\%. Shown on the right side are slices through the center of the domain together with the RBC boundaries in the initial and final configuration. The full simulation video is available at https://vimeo.com/329509435.

- BIE-FMM: FMM calls required to evaluate $\boldsymbol{u}^{\Gamma}$ at the check points and at points on RBCs

- Other-FMM: FMM calls required by other algorithms

- Other: all other operations

In the discussion below, we focus on $\mathbf{C O L}$ and BIE-solve, as they are the primary algorithmic contribution of this work, and discuss how to reduce the computational time required for $\boldsymbol{B I E - F M M}$.

Strong scalability. To study the strong scalability of our algorithms, we use the blood vessel geometry and RBC configuration in Fig. 1-left. This simulation contains 40,960 RBCs and the blood vessel is represented with 40,960 patches. With four degrees of freedom per RBC quadrature point and three per vessel quadrature point, this amounts to $89,128,960$ and $14,868,480$ degrees for the RBCs and blood vessel, respectively (103,997,440 in total). As can be seen from Fig. 4, we achieve a 15.7-fold speed-up in total walltime scaling from 384 to 12288 cores, corresponding to $49 \%$ parallel efficiency. This level of parallel efficiency is partially due to the calls to the fmm library PVFMM. The strong scalability of PVFMM we observe is largely consistent with the results reported in [27]. Neglecting the time for calls to FMM, i.e., only counting the time for the boundary solver to compute $\boldsymbol{u}^{\Gamma}$ and for collision prevention, we find $66 \%$ parallel efficiency when scaling strongly from 384 to 12288 cores. We see that the parallel collision handling and integral 


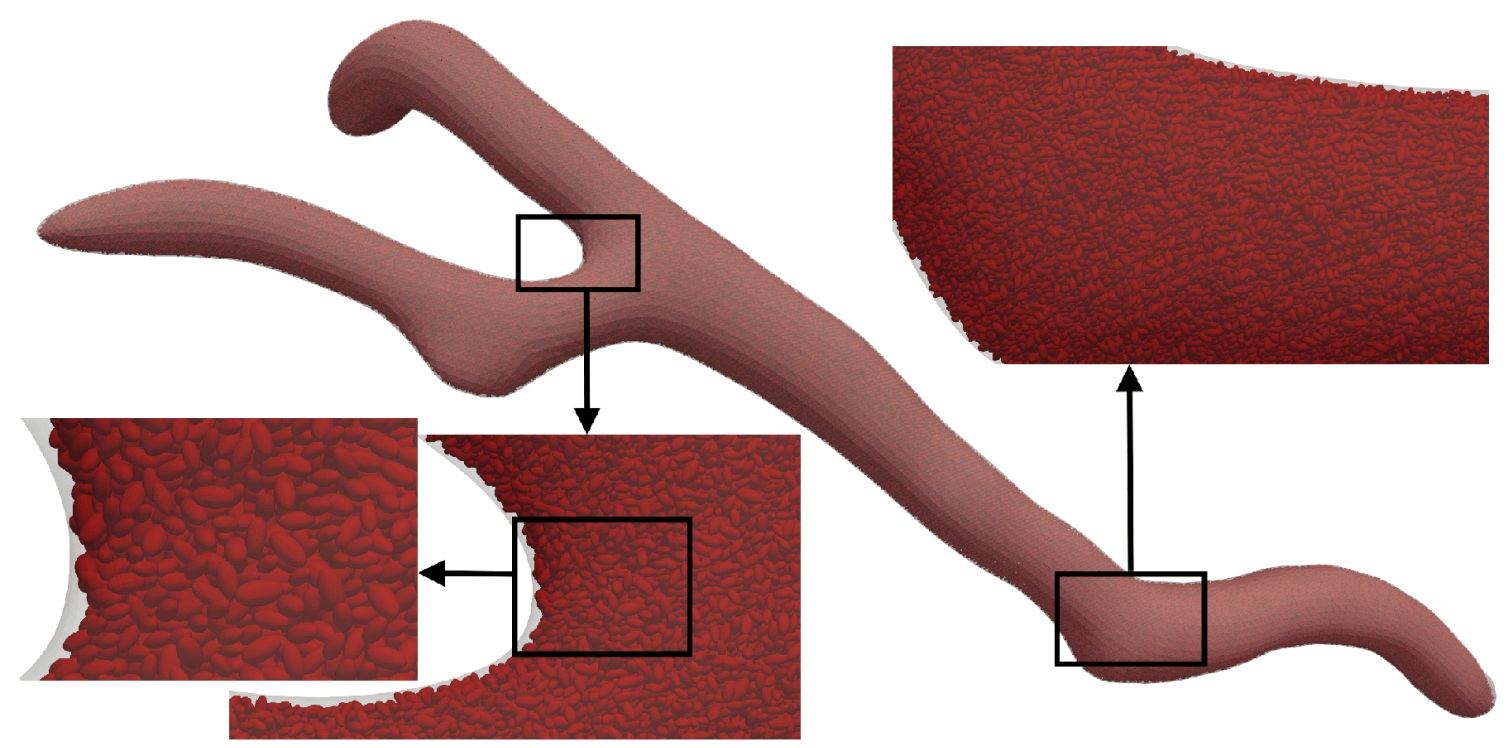

Figure 8: For our weak scaling experiments, we use the the vessel geometry shown above with inflow boundary conditions on the right side and outflow boundary condition on the two left sides. To setup the problem, we fill the vessel with nearly-touching RBCs of different sizes to obtain a desired number, and refine the vessel geometry patches. The figure above shows a setup with overall 262,144 RBCs at a volume fraction of $26 \%$.

equation solver computations, excluding FMM, scale well as the number of cores is increased.

Weak Scalability. Our weak scalability results are shown in Figs. 5 and 6. Both tests are performed on the blood vessel displayed in Fig. 8. We use an initial boundary composed of a fixed number $M$ of polynomial patches and fill the domain with roughly $M / 2$ RBCs (which requires spacing $h$ ). To scale up our simulation by a factor of four, we: (1) subdivide the $M$ polynomial patches into $4 M$ new but equivalent polynomial patches (via subdivision rules for Bezier curves); (2) refill the domain with RBCs using spacing $h / \sqrt[3]{4}$. This places $2 M \mathrm{RBCs}$ in the domain volume. We repeat this process each time we increase the number of cores by a factor of four in order to keep the number of patches and RBCs per core constant. In the tables in Figs. 5 and 6, we report parallel efficiency with respect to the first multi-node run on both SKX and KNL architectures, i.e, with respect to 192 and 136 cores, respectively.

The largest weak scaling test contains 1,048,576 RBCs and 2,097,152 polynomial patches on the blood vessel; we solve for 3,042,967,552 unknowns at each time step and are able to maintain a collisionfree state between 4,194,304,000 triangular surface elements at each time step. Comparing the weak scalability results for SKX (Fig. 5) and KNL (Fig. 6), we observe similar qualitative behavior. Note that the smallest test on the SKX architecture only uses a single node, i.e., no MPI communication was needed. This explains the increased time for the collision prevention algorithms when going from 1 (48 cores) to 4 nodes (192 cores). Note also that the simulation on the KNL architecture used a significantly lower number of RBCs and geometry patches per node. Thus, this simulation has a larger ratio of communication to local work. This explains the less perfect scalability compared to the results obtained on the SKX architecture. As with strong scaling, we see good parallel scaling of the non-FMM-related parts of the computation of $\boldsymbol{u}^{\Gamma}$ and the collision handling algorithm.

Note that there is a slight variation in the number of collisions for the run on 8704 cores on KNL. This is an artifact of the RBC filling algorithm. Since we place RBCs in random orientations and distribute RBCs randomly among processors, we do not have complete control over the percentage of collisions or the volume fraction for each simulation in Figs. 5 and 6, as can be seen from the tables under these figures. This can affect the overall scaling: For the run on 8704 cores, the percentage of collisions is larger, explaining the longer time spent in $\mathbf{C O L}$. Despite this phenomenon, we achieve good weak scaling overall.

Discussion. The parts of the algorithm introduced in this paper scale as well as or better than the FMM implementation we are using. However, our overall run time is diminished by the multiple expensive FMM evaluations required for solving Eq. (2.5). This can be addressed by using a local singular quadrature scheme, i.e., compute a singular integral using the FMM on Eq. (3.1) directly, then compute a singular correction locally. This calculation has a three-fold impact on parallel scalability: (1) the FMM evaluation required is proportional to the size of the coarse discretization rather than the fine discretization $(O((p+1) N)$ vs. $O((k+p) N))$; (2) after the FMM evaluation, the local correction is embarrassingly parallel; (3) the linear operator Eq. (3.3) can be precomputed, making the entire calculation extremely fast with MKL linear algebra routines. These improvements together will allow our algorithm to scale well beyond the computational regime explored in this work. 


\subsection{Verification}

There are few analytic results known about RBCs in confined Stokes flows against which we can verify our simulations. However, exact solutions can be obtained for a part of our setup, invariants (e.g., surface area) can be considered and solutions for smaller examples can be verified against solutions with fine spatial and temporal discretizations. In particular, in this section, we demonstrate the accuracy of the parallel boundary solver presented in Section 3 and numerically study the collision-free time-stepping in Section 4.

Boundary solver. The error of the boundary integral solver is determined by the error of integration and the GMRES error, the latter not depending on the number of discretization points due to good conditioning of the equation. The integration error, in turn, can be separated into smooth quadrature error and interpolation error. The former is high-order accurate [46]. Although our extrapolation is ill-conditioned, we observe good accuracy for $p \leq 8$. The singular evaluation in Section 3 converges with rate $O\left(L^{p}+L^{q}\right)$ corresponding to $p$ th order extrapolation and $q$ th order quadrature. To confirm this numerically, we solve an interior Stokes problem on the surface in Fig. 9-right. We evaluate a prescribed analytic solution at the discretization points to obtain the boundary condition. We then solve Eq. (3.5) and compare the numerical solution at on-surface samples different from discretization points, evaluated using the algorithms of Section 3. We use $\eta=2, q=16, p=8$, $R=.04 \sqrt{L}$ and $r=R / 8$. In Fig. 9-left, we report the relative error in the infinity-norm of the velocity. By choosing check point distances proportional to $\sqrt{L}$, we observe the expected $O\left(L^{7}\right)$ convergence.
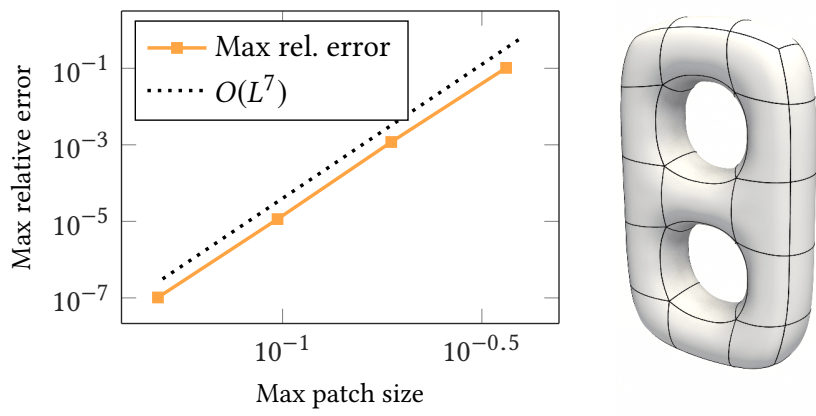

Figure 9: Error convergence test solving Eq. (3.5). We evaluate a known solution on the coarse discretization and solve for $\phi$. On the left, we plot the maximum relative error in the infinity norm of $\boldsymbol{u}^{\Gamma}$ evaluated on the surface. On the right, we show the coarse discretization of the domain boundary and patches.

RBCs with collision resolution and convergence. Our choice of $\mathrm{RBC}$ representation and discretization is spectrally accurate in space for the approximation, differentiation and integration of functions on RBC surfaces, as shown in [48]. Although we use first-order time-stepping in this work, spectral deferred correction (SDC) can be incorporated into the algorithm exactly as in the $2 \mathrm{D}$ version described in [24]. This present work demonstrates second-order convergence in time; however, SDC can be made arbitrarily highorder accurate.

For collision-resolution accuracy verification, we study the convergence of our contact-free time-stepping with two RBCs in shear

flow. As shown in Fig. 10, at $T=0$, two RBCs are placed in a shear flow $\boldsymbol{u}=[z, 0,0]$ in free-space. We first compute a reference solution without collision handling but with expensive adaptive fully implicit time-stepping to ensure accurate resolution of the lubrication layer between RBCs. This reference simulatation used spherical harmonics of order 32 and the time step had to be reduced to $6.5 \mathrm{e}-4$ to prevent collisions. In Fig. 11, we show the convergence for the error in the centers of mass of each RBC as a function of the time-step size. We use spherical harmonic orders 16 and 32 for the spatial discretization to demonstrate the dominance of the time-stepping error. We observe first-order convergence with our locally-implicit backward Euler scheme which confirms that our collision resolution algorithm does not have a significant impact on time-stepping accuracy.

(a) $t=0$

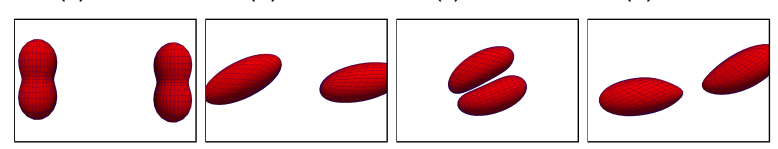

Figure 10: Snapshots of two vesicles in shear flow.

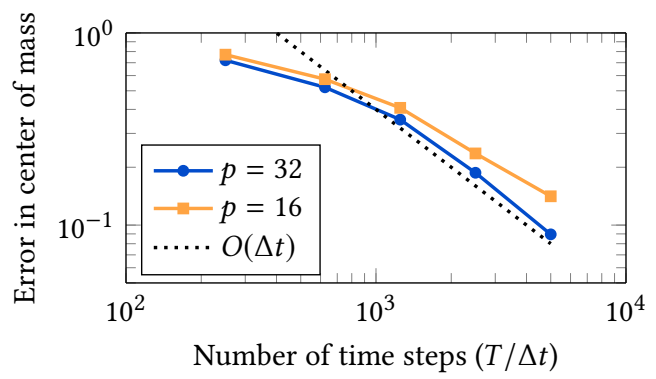

Figure 11: Shown is the error in the final $(T=25)$ centroid location as we decrease the time step size for two spherical harmonic orders 16 and 32. We observe $O(\Delta t)$ convergence in time and hence the collission detection algorithm converges at the same order as the time stepper.

\subsection{High volume fraction}

The RBC volume fraction, i.e., the ratio of volume occupied by RBCs compared to the overall blood volume is $36-48 \%$ in healthy women and $40-54 \%$ in healthy men [5]. As can be seen in the tables in Figs. 5 and 6 , the volume fraction in our weak scaling simulations is below these values, which is mostly due to the procedure used to fill the blood vessel with RBCs (see the discussion in Section 5.1). However, $\mathrm{RBC}$ volume fractions in capillaries and small arteries is known to be be around $10-20 \%$ [41, 51], which our scaling simulations achieve. To demonstrate that we can simulate even higher volume fraction blood flows, Fig. 7 shows a test of 140 RBCs sedimenting under a gravitational force in a small capsule. The volume fraction for this example is $47 \%$, calculated by dividing the amount of volume occupied by RBCs by the volume of the capsule. By the end of the simulation, we achieve a volume fraction of $55 \%$ in the lower part of the domain (determined by bounding the RBCs by a tighter cylinder 
than the original domain boundary) since the RBCs have become more tightly packed. While such high volume fractions typically do not occur in capillary flow on average, in some scenarios (local fluctuations, sedimentation, microfluidics) these high concentrations need to be handled.

\section{CONCLUSION}

We have shown that our parallel platform for the simulation of red blood cell flows is capable of accurately resolved long-time simulation of red blood cell flows in complex vessel networks. We are able to achieve realistic cell volume fractions of over $47 \%$, while avoiding collisions between cells or with the blood vessel walls. Incorporating blood vessels into red blood cell simulations requires solving a boundary integral equation, for which we use GMRES Each GMRES iteration computes a matrix-vector product, which in turn involves singular quadrature and an FMM evaluation; the latter dominates the computation time. To avoid collisions, we solve a nonlinear complementarity problem in the implicit part of each time step. This requires repeated assembly of sparse matrices that, in principle, couple all cells globally. Nevertheless, solving this complementarity system yields close-to-optimal strong and weak scaling in our tests. Overall, the vast majority of compute time is spent in FMM evaluations, which implies that the scaling behavior of our simulation is dominated by the scalability of the FMM implementation. As discussed at the end of Section 5.2, in the future, we will employ a local singular quadrature scheme that will allow us to significantly reduce the time spent in FMM evaluations. This will not only speed up the overall simulation but also improve the weak and strong scalability of our simulation platform.

\section{ACKNOWLEDGEMENTS}

We would like to thank Dhairya Malhotra, Michael Shelley and Shenglong Wang for support and various discussions throughout about various aspects of this work. This work was supported by the US National Science Foundation (NSF) through grants DMS-1821334, DMS-1821305, DMS-1320621, DMS-1436591 and EAR-1646337. Computing time on TACC's Stampede2 supercomputer was provided through the Extreme Science and Engineering Discovery Environment (XSEDE), which is supported by National Science Foundation grant number ACI-1548562. 


\section{REFERENCES}

[1] N. Al Quddus, W. A. Moussa, and S. Bhattacharjee. "Motion of a spherical particle in a cylindrical channel using arbitrary LagrangianEulerian method". In: fournal of colloid and interface science 317.2 (2008), pp. 620-630.

[2] S. Balay, S. Abhyankar, M. Adams, J. Brown, P. Brune, K. Buschelman, L. Dalcin, V. Eijkhout, W Gropp, D. Kaushik, et al. Petsc users manual revision 3.8. Tech. rep. Argonne National Lab.(ANL), Argonne, IL (United States), 2017.

[3] P. Balogh and P. Bagchi. "A computational approach to modeling cellular-scale blood flow in complex geometry”. In: fournal of Computational Physics 334 (2017), pp. 280-307.

[4] P. Balogh and P. Bagchi. "Direct numerical simulation of cellularscale blood flow in 3D microvascular networks". In: Biophysical journal 113.12 (2017), pp. 2815-2826.

[5] H. H. Billett. "Hemoglobin and hematocrit". In: Clinical Methods: The History, Physical, and Laboratory Examinations. 3rd edition. Butterworths, 1990.

[6] O. P. Bruno and S. K. Lintner. "A high-order integral solver for scalar problems of diffraction by screens and apertures in threedimensional space”. In: fournal of Computational Physics 252 (2013), pp. 250-274.

[7] C. Burstedde, L. C. Wilcox, and O. Ghattas. "p4est: Scalable Algorithms for Parallel Adaptive Mesh Refinement on Forests of Octrees". In: SIAM fournal on Scientific Computing 33.3 (2011), pp. 1103-1133. DOI: $10.1137 / 100791634$.

[8] P. B. Canham. "The minimum energy of bending as a possible explanation of the biconcave shape of the human red blood cell". In: fournal of theoretical biology 26.1 (1970), pp. 61-81.

[9] C. G. Caro, T. Pedley, R. Schroter, and W. Seed. The mechanics of the circulation. Cambridge University Press, 2012.

[10] P. Du, J. Zhao, W. Cao, and Y. Wang. "DCCD: Distributed N-Body Rigid Continuous Collision Detection for Large-Scale Virtual Environments". In: Arabian fournal for Science and Engineering 42.8 (2017), pp. 3141-3147.

[11] S. Fang. "A linearization method for generalized complementarity problems”. In: IEEE transactions on automatic control 29.10 (1984), pp. 930-933.

[12] J. B. Freund. "Numerical simulation of flowing blood cells". In: Annual review of fluid mechanics 46 (2014), pp. 67-95.

[13] J. Gounley, M. Vardhan, and A. Randles. "A Computational Framework to Assess the Influence of Changes in Vascular Geometry on Blood Flow". In: Proceedings of the Platform for Advanced Scientific Computing Conference. ACM. 2017, p. 2.

[14] I. G. Graham and I. H. Sloan. "Fully discrete spectral boundary integral methods for Helmholtz problems on smooth closed surfaces in $\mathbb{R}^{3}$. In: Numerische Mathematik 92.2 (2002), pp. 289-323.

[15] L. Greengard and V. Rokhlin. "A fast algorithm for particle simulations”. In: fournal of computational physics 73.2 (1987), pp. 325348.

[16] L. Grinberg, J. A. Insley, V. Morozov, M. E. Papka, G. E. Karniadakis, D. Fedosov, and K. Kumaran. "A new computational paradigm in multiscale simulations: Application to brain blood flow”. In: Proceedings of 2011 International Conference for High Performance Computing, Networking, Storage and Analysis. ACM. 2011, p. 5.

[17] D. Harmon, D. Panozzo, O. Sorkine, and D. Zorin. "Interferenceaware geometric modeling”. In: ACM Transactions on Graphics 30.6 (Dec. 2011), p. 1.
[18] W. Helfrich. "Elastic properties of lipid bilayers: theory and possible experiments". In: Zeitschrift für Naturforschung C 28.11-12 (1973), pp. 693-703.

[19] K. Iglberger and U. Rüde. "A Parallel Rigid Body Dynamics Algorithm”. In: Euro-Par 2009 Parallel Processing. Ed. by H. Sips, D. Epema, and H.-X. Lin. Berlin, Heidelberg: Springer Berlin Heidelberg, 2009, pp. 760-771.

[20] D. Kim, J.-P. Heo, J. Huh, J. Kim, and S.-e. Yoon. "HPCCD: Hybrid Parallel Continuous Collision Detection using CPUs and GPUs". In: Computer Graphics Forum (2009).

[21] L. af Klinteberg and A.-K. Tornberg. "A fast integral equation method for solid particles in viscous flow using quadrature by expansion". In: fournal of Computational Physics 326 (2016), pp. 420-445.

[22] A. Klöckner, A. Barnett, L. Greengard, and M. O’Neil. "Quadrature by expansion: A new method for the evaluation of layer potentials". In: Journal of Computational Physics 252 (2013), pp. 332-349.

[23] F. Liu, T. Harada, Y. Lee, and Y. J. Kim. "Real-time Collision Culling of a Million Bodies on Graphics Processing Units". In: ACM SIGGRAPH Asia 2010 Papers. SIGGRAPH ASIA '10. Seoul, South Korea: ACM, 2010, 154:1-154:8.

[24] L. Lu, A. Rahimian, and D. Zorin. "Contact-aware simulations of particulate Stokesian suspensions”. In: fournal of Computational Physics 347C (Nov. 2017), pp. 160-182. DoI: 10.1016/j.jcp.2017.06.039. arXiv: 1612.02057 .

[25] L. Lu, A. Rahimian, and D. Zorin. "Parallel contact-aware simulations of deformable particles in 3D Stokes flow". In: arXiv preprint arXiv:1812.04719 (2018).

[26] D. Malhotra and G. Biros. "PVFMM: A Parallel Kernel Independent FMM for Particle and Volume Potentials". In: Communications in Computational Physics 18 (2015), pp. 808-830.

[27] D. Malhotra and G. Biros. "Algorithm 967: A distributed-memory fast multipole method for volume potentials”. In: ACM Transactions on Mathematical Software (TOMS) 43.2 (2016), p. 17.

[28] D. Malhotra, A. Rahimian, D. Zorin, and G. Biros. "A parallel algorithm for long-timescale simulation of concentrated vesicle suspensions in three dimensions". In: (2017).

[29] H. Mazhar, T. Heyn, and D. Negrut. "A scalable parallel method for large collision detection problems”. In: 26 (June 2011), pp. 37-55.

[30] E. Nazockdast, A. Rahimian, D. Zorin, and M. Shelley. "Fast and highorder methods for simulating fiber suspensions applied to cellular mechanics". preprint. 2015.

[31] S. Pabst, A. Koch, and W. Strasser. "Fast and Scalable CPU/GPU Collision Detection for Rigid and Deformable Surfaces”. In: Computer Graphics Forum (2010).

[32] P. Perdikaris, L. Grinberg, and G. E. Karniadakis. "An effective fractaltree closure model for simulating blood flow in large arterial networks". In: Annals of biomedical engineering 43.6 (2015), pp. 14321442.

[33] P. Perdikaris, L. Grinberg, and G. E. Karniadakis. "Multiscale modeling and simulation of brain blood flow". In: Physics of Fluids 28.2 (2016), p. 021304.

[34] M. Peyrounette, Y. Davit, M. Quintard, and S. Lorthois. "Multiscale modelling of blood flow in cerebral microcirculation: Details at capillary scale control accuracy at the level of the cortex". In: PloS one 13.1 (2018), e0189474.

[35] H. Power and G. Miranda. "Second kind integral equation formulation of Stokes' flows past a particle of arbitrary shape". In: SIAM fournal on Applied Mathematics 47.4 (1987), p. 689. 
[36] C. Pozrikidis. Boundary integral and singularity methods for linearized viscous flow. Cambridge Texts in Applied Mathematics. Cambridge University Press, Cambridge, 1992.

[37] A. Rahimian, I. Lashuk, S. Veerapaneni, A. Chandramowlishwaran, D. Malhotra, L. Moon, R. Sampath, A. Shringarpure, J. Vetter, R. Vuduc, et al. "Petascale direct numerical simulation of blood flow on $200 \mathrm{k}$ cores and heterogeneous architectures". In: Proceedings of the 2010 ACM/IEEE International Conference for High Performance Computing, Networking, Storage and Analysis. IEEE Computer Society. 2010, pp. 1-11.

[38] A. Rahimian, S. K. Veerapaneni, D. Zorin, and G. Biros. "Boundary integral method for the flow of vesicles with viscosity contrast in three dimensions". In: Journal of Computational Physics 298 (2015), pp. 766-786.

[39] A. Randles, E. W. Draeger, T. Oppelstrup, L. Krauss, and J. A. Gunnels. "Massively parallel models of the human circulatory system". In: Proceedings of the International Conference for High Performance Computing, Networking, Storage and Analysis. ACM. 2015, p. 1.

[40] D. Rossinelli, Y.-H. Tang, K. Lykov, D. Alexeev, M. Bernaschi, P. Hadjidoukas, M. Bisson, W. Joubert, C. Conti, G. Karniadakis, et al. "The in-silico lab-on-a-chip: petascale and high-throughput simulations of microfluidics at cell resolution". In: Proceedings of the International Conference for High Performance Computing, Networking, Storage and Analysis. ACM. 2015, p. 2.

[41] A. Saadat, C. J. Guido, and E. S. Shaqfeh. "Simulation of Red Blood Cell Migration in Small Arterioles: Effect of Cytoplasmic Viscosity". In: bioRxiv (2019), p. 572933.

[42] A. Saadat, C. J. Guido, G. Iaccarino, and E. S. Shaqfeh. "Immersedfinite-element method for deformable particle suspensions in viscous and viscoelastic media”. In: Physical Review E 98.6 (2018), p. 063316.

[43] C. Sorgentone and A.-K. Tornberg. "A highly accurate boundary integral equation method for surfactant-laden drops in 3D”. In: fournal of Computational Physics 360 (2018), pp. 167-191.

[44] C. Sorgentone, A.-K. Tornberg, and P. M. Vlahovska. "A 3D boundary integral method for the electrohydrodynamics of surfactant-covered drops". In: Journal of Computational Physics (2019).

[45] H. Sundar, D. Malhotra, and G. Biros. "HykSort: A New Variant of Hypercube Quicksort on Distributed Memory Architectures". In: Proceedings of the 27th International ACM Conference on International Conference on Supercomputing. ICS '13. Eugene, Oregon, USA: ACM, 2013, pp. 293-302.

[46] L. N. Trefethen. Approximation theory and approximation practice. Vol. 128. Siam, 2013.

[47] S. K. Veerapaneni, D. Gueyffier, G. Biros, and D. Zorin. "A numerical method for simulating the dynamics of 3D axisymmetric vesicles suspended in viscous flows". In: Fournal of Computational Physics 228.19 (Apr. 2009), pp. 7233-7249.

[48] S. K. Veerapaneni, A. Rahimian, G. Biros, and D. Zorin. "A fast algorithm for simulating vesicle flows in three dimensions". In: fournal of Computational Physics 230.14 (2011), pp. 5610-5634.

[49] M. Wala and A. Klöckner. "A Fast Algorithm with Error Bounds for Quadrature by Expansion”. In: arXiv preprint arXiv:1801.04070 (2018).

[50] M. Wala and A. Klöckner. "Optimization of Fast Algorithms for Global Quadrature by Expansion Using Target-Specific Expansions”. In: arXiv preprint arXiv:1811.01110 (2018).
[51] W. Wang, T. G. Diacovo, J. Chen, J. B. Freund, and M. R. King. "Simulation of platelet, thrombus and erythrocyte hydrodynamic interactions in a 3D arteriole with in vivo comparison". In: PLoS One 8.10 (2013), e76949.

[52] D. Xu, E. Kaliviotis, A. Munjiza, E. Avital, C. Ji, and J. Williams. "Large scale simulation of red blood cell aggregation in shear flows". In: Journal of Biomechanics 46.11 (2013), pp. 1810-1817.

[53] W. Yan, H. Zhang, and M. J. Shelley. "Computing collision stress in assemblies of active spherocylinders: Applications of a fast and generic geometric method". In: The Journal of chemical physics 150.6 (2019), p. 064109.

[54] T. Ye, L. Peng, and Y. Li. "Three-dimensional motion and deformation of a red blood cell in bifurcated microvessels". In: Journal of Applied Physics 123.6 (2018), p. 064701.

[55] T. Ye, N. Phan-Thien, and C. T. Lim. "Particle-based simulations of red blood cellsâĂŤA review”. In: Journal of biomechanics 49.11 (2016), pp. 2255-2266.

[56] T. Ye, N. Phan-Thien, C. T. Lim, L. Peng, and H. Shi. "Hybrid smoothed dissipative particle dynamics and immersed boundary method for simulation of red blood cells in flows". In: Physical Review E 95.6 (2017), p. 063314.

[57] L. Ying, G. Biros, and D. Zorin. "A high-order 3D boundary integral equation solver for elliptic PDEs in smooth domains”. In: Journal of Computational Physics 219.1 (2006), pp. 247-275.

[58] L. Ying, G. Biros, and D. Zorin. "A high-order 3D boundary integral equation solver for elliptic PDEs in smooth domains”. In: fournal of Computational Physics 219.1 (2006), pp. 247-275.

[59] H. Zhao, A. H. Isfahani, L. N. Olson, and J. B. Freund. "A spectral boundary integral method for flowing blood cells". In: Journal of Computational Physics 229.10 (May 2010), pp. 3726-3744. 


\section{Appendix: Artifact Description/Artifact Evaluation}

\section{SUMMARY OF THE EXPERIMENTS REPORTED}

We ran our weak and strong scalability tests on Stampede2 system at the Texas Advanced Computing Center (TACC) with the Knights Landing (KNL) compute 788 nodes and the Skylake (SKX) compute nodes. I use intel 18.0.2, Intel MPI 18.0.2, PETSc 3.10, p4est 2.0, FFTW 3 3.3.8, boost 1.68, PVFMM libraries for the simulation tests.

We use the Stampede2 system at the Texas Advanced Computing Center (TACC) to study the scalability of our algorithms and implementation. Stampede2 has two types of compute nodes, the Knights Landing (KNL) compute nodes and the Skylake (SKX) compute nodes. The SKX cluster has 1,736 dual-socket compute nodes, each with two 24-core $2.1 \mathrm{GHz}$ CPUs and 192GB of memory. The KNL cluster has 4,200 compute nodes, with a 68-core Intel Xeon Phi 7250 1.4Ghz CPUs and 96GB of memory plus 16GB of highspeed MCDRAM. We run our simulations in a hybrid distributedshared memory fashion: we run one MPI process per node, with one OpenMP thread per hardware core. Our largest simulations use 256 SKX and 512 KNL nodes.

We leverage several high-performance libraries in our implementation. We use PETSc's parallel matrix and vector operations, and its parallel GMRES solver. Management and distribution of patches describing the blood vessel geometry uses the p4est library, and we use PVFMM for parallel FMM evaluation. We also heavily leverage Intel MKL for fast dense linear algebra routines at the core of our algorithms and paraview for our visualizations.

Our largest run on Stampede2 finished at 2019-04-08 01:38:16(US central time), the date range of access to Stampede 2 for all tests is around 2019-03-20 to 2019-04-09.

\section{ARTIFACT AVAILABILITY}

Software Artifact Availability: Some author-created software artifacts are NOT maintained in a public repository or are NOT available under an OSI-approved license.

Hardware Artifact Availability: There are no author-created hardware artifacts.

Data Artifact Availability: Some author-created data artifacts are NOT maintained in a public repository or are NOT available under an OSI-approved license.

Proprietary Artifacts: None of the associated artifacts, authorcreated or otherwise, are proprietary.

List of URLs and/or DOIs where artifacts are available:

pvfmm "https://github.com/dmalhotra/pvfmm"

petsc "https://www.mcs.anl.gov/petsc/"

p4est "http://www.p4est.org/"

intel-parallel-studio "https://software.intel.com/en」

$\hookrightarrow$-us/articles/intel-c-compiler-180-for-linux-relea 」

$\hookrightarrow$ se-notes-for-intel-parallel-studio-xe-2018"

fftw3 "http://www.fftw.org/"

boost "https://www. boost.org/users/history/version $\backslash_{-}$ $\hookrightarrow \quad 1 \backslash$ 68\_0.html"

\section{BASELINE EXPERIMENTAL SETUP, AND MODIFICATIONS MADE FOR THE PAPER}

Relevant hardware details: Stampede2 skylake compute nodes and Knights Landing compute nodes

Operating systems and versions: Linux version 3.10.0957.5.1.el7.x86_64

Compilers and versions: intel 18.0.2, impi 18.0.2

Libraries and versions: intel 18.0.2, impi 18.0.2, eigen 3.3.1, CGAL 4.10, fftw3 3.3.8, boost 1.68 , vtk 8.1.1, pvfmm, p4est 2.0, petsc 3.10i 64

Key algorithms: fast multipole method, boundary integral equation method tion.

Output from scripts that gathers execution environment informa-

On Stampde2 skx nodes, the execution environment

$\hookrightarrow$ information is the following:

c506-033[skx](508)\$./collect_environment.sh

SLURM_NODELIST $=c 506-033$

SLURM_CHECKPOINT_IMAGE_DIR=/var/slurm/checkpoint

LMOD_FAMILY_COMPILER_VERSION $=18.0 .2$

MKLROOT=/opt/intel/compilers_and_libraries_2018.2.19」

$\hookrightarrow \quad 9 / 1$ inux $/ \mathrm{mkl}$

I_MPI_STARTUP_MODE=pmi_shm_netmod

P4EST_DIR=/home1/apps/intel18/impi18_o/p4est/2.0

SLURM_JOB_NAME $=i d v 31559$

MANPATH=/opt/apps/libfabric/1.7.0/share/man:/opt/int 」

$\hookrightarrow$ el/compilers_and_libraries_2018.2.199/linux/mpi/ 」

$\hookrightarrow$ man:/opt/intel/documentation_2018/en/man/common:

$\hookrightarrow$ /opt/intel/documentation_2018/en/debugger/gdb-ig 」

$\hookrightarrow \mathrm{fx} / \mathrm{man}$ :/opt/intel/documentation_2018/en/debugger 」

$\hookrightarrow$ /gdb-ia/man:/opt/apps/intel18/impi18_0/fftw3/3.3」

$\hookrightarrow .8 / \mathrm{man}$

TACC_IMPI_DIR=/opt/intel/compilers_and_libraries_201

$\hookrightarrow$ 8.2.199/linux/mpi

TACC_FFTW3_INC=/opt/apps/intel18/impi18_0/fftw3/3.3.」

$\hookrightarrow \quad 8 /$ include

VTK_DIR=/home1/apps/intel18/impi18_0/vtk/8.1.1

XDG_SESSION_ID $=283415$

TACC_BOOST_INC=/opt/apps/intel18/boost/1.68/include

HOSTNAME $=c 506-033$

SLURMD_NODENAME $=c 506-033$

SLURM_TOPOLOGY_ADDR $=c 506-033$

TACC_INTEL_INC=/opt/intel/compilers_and_libraries_20

$\hookrightarrow$ 18.2.199/linux/compiler/include/intel64 
_ModuleTable003_=LjAuMi IsWyJsb2FkT3JkZXIiXT0xLHByb3B 」 $\hookrightarrow \quad$ UPXt9LFsic3RhY2tEZXB0aCJdPTAsWyJzdGFOdXMiXT0iYWN 」 $\hookrightarrow$ OaXZlIixbInVzZXJOYW1lIl09ImludGVsIix9LGxpYmZhYnJ」 $\hookrightarrow \quad$ pYz17WyJmbiJdPSIvb3B0L2FwcHMvbW9kdWxIZmlsZXMvbGl」 $\hookrightarrow \quad i Z m F i c m l j L z E u N y 4 w L m x 1 Y S I s W y J m d W x s T m F t Z S J d P S J s a W J$ 」 $\hookrightarrow \quad$ mYWJyaWMvMS43LjAiLFsibG9hZE9yZGVyI109Mixwcm9wVD1 」

$\hookrightarrow$ 7fSxbInJ1Z19jb3VudCJdPTEsWyJzdGFja0RIcHRoIl09MSx ।

$\hookrightarrow \quad$ bInNOYXR1cyJdPSJhY3RpdmUiLFsidXNlck5hbWUiXT0ibGl」

$\hookrightarrow \quad$ iZmFicmljIix9LHA0ZXNOPXtbImZuI109Ii 9vcHQvYXBwcy9」

$\hookrightarrow$ pbnRIbDE4L21tcGkxOF8wL21vZHVsZWZpbGVzL3A0ZXNOLzI 」

$\hookrightarrow \quad$ UMC5sdWEiLFsiZnVsbE5hbWUiXT0icDRlc3QvMi 4wIixb

TACC_FAMILY_QT_VERSION=5.11.2

SLURM_PRIO_PROCESS $=0$

SLURM_NODE_ALIASES $=($ null $)$

INTEL_LICENSE_FILE=/home1/anonymous/USER/intel/licen 」

$\hookrightarrow$ ses:/opt/intel/licenses:/opt/intel/compilers_and 」

$\hookrightarrow$ _libraries_2018.2.199/linux/licenses

IPPROOT=/opt/intel/compilers_and_libraries_2018.2.19」

$\hookrightarrow \quad$ 9/linux/ipp

I_MPI_F77=ifort

MPICH_HOME=/opt/intel/compilers_and_libraries_2018.2」

$\hookrightarrow \quad .199 /$ linux/mpi

TACC_PETSC_BIN=/home1/apps/intel18/impi18_0/petsc/3. 」 $\hookrightarrow 10 /$ skylake-i64/bin

SHELL=/bin/bash

TERM=xterm-256color

__LMOD_REF_COUNT_MODULEPATH=/opt/apps/qt5.11.2/modul 」

$\hookrightarrow$ efiles:1;/opt/apps/intel18/impi18_0/modulefiles: 」

$\hookrightarrow 1$;/opt/apps/intel18/modulefiles: 1 ;/opt/apps/xsed 」

$\hookrightarrow$ e/modulefiles:1;/opt/apps/modulefiles:1;/opt/mod 」

$\hookrightarrow$ ulefiles: 1

NO_HOSTSORT $=1$

TACC_INTEL_DIR=/opt/intel/compilers_and_libraries_20 」 $\hookrightarrow$ 18.2.199/linux

TACC_LIBFABRIC_BIN=/opt/apps/libfabric/1.7.0/bin

TACC_FAMILY_QT=qt5

FFTW_ROOT=/opt/apps/intel18/impi18_0/fftw3/3.3.8

HISTSIZE $=1000$

IDEV_SETUP_BYPASS $=1.0$

SLURM_JOB_QOS=normal

I_MPI_FABRICS $=$ shm: of $i$

TACC_IMPI_BIN=/opt/intel/compilers_and_libraries_201」

$\hookrightarrow$ 8.2.199/linux/mpi/intel64/bin

VES3D_DIR=/home1/anonymous/USER/projects/boundary/ve 」

$\hookrightarrow$ S3d-CXX

SSH_CLIENT=206.76.192.52 6061022

LMOD_SYSTEM_DEFAULT_MODULES=TACC

TMPDIR $=/ \mathrm{tmp}$

SLURM_TOPOLOGY_ADDR_PATTERN=node

TACC_LIBFABRIC_DIR=/opt/apps/libfabric/1.7.0

PETSC_ARCH=skylake- $i 64$

QT_QPA_PLATFORM_PLUGIN_PATH=/opt/apps/qt5/5.11.2/plu 」 $\hookrightarrow$ gins

XALT_DIR=/opt/apps/xalt/xalt/
LIBRARY_PATH=/opt/intel/compilers_and_libraries_2018」 $\hookrightarrow$.2.199/linux/daal/../tbb/lib/intel64_lin/gcc4.4: 」

$\hookrightarrow$ /opt/intel/compilers_and_libraries_2018.2.199/1i 」

$\hookrightarrow$ nux/daal/lib/intel64_lin:/opt/intel/compilers_an」

$\hookrightarrow$ d_libraries_2018.2.199/linux/tbb/lib/intel64/gcc 」

$\hookrightarrow \quad 4.7:$ /opt/intel/compilers_and_libraries_2018.2.19」

$\hookrightarrow$ 9/linux/mkl/lib/intel64_lin:/opt/intel/compilers 」

$\hookrightarrow$ _and_libraries_2018.2.199/linux/compiler/lib/int 」

$\hookrightarrow$ el64_lin:/opt/intel/compilers_and_libraries_2018」

$\hookrightarrow \quad .2 .199 /$ linux/ipp/lib/intel64

TACC_LIBFABRIC_INC=/opt/apps/libfabric/1.7.0/include

TACC_BOOST_BIN=/opt/apps/intel18/boost $/ 1.68 / \mathrm{bin}$

LMOD_PKG=/opt/apps/1mod/1mod

TACC_FAMILY_COMPILER_VERSION $=18.0 .2$

TACC_FAMILY_VTK_VERSION=8.1.1

QTDIR=/opt/apps/qt5/5.11.2

TACC_P4EST_BIN=/home1/apps/intel18/impi18_0/p4est/2. 」 $\hookrightarrow \quad 0 / \mathrm{bin}$

QTINC=/usr/lib64/qt-3.3/include

LMOD_VERSION=7.8.21

SSH_TTY $=/$ dev $/ p t s / 0$

SLURM_TACC_RUNLIMIT_MINS $=30$

__LMOD_REF_COUNT_LOADEDMODULES=intel/18.0.2:1; libfab 」

$\hookrightarrow \mathrm{ric} / 1.7 .0: 1 ; \mathrm{impi} / 18.0 .2: 1 ; \mathrm{fftw} 3 / 3.3 .8: 1 ; \mathrm{petsc} / 3$.

$\hookrightarrow$ 10-i64:1;boost/1.68:1;qt5/5.11.2:1;vtk/8.1.1:1; 」

$\hookrightarrow$ 4est/2.0:1

I_MPI_JOB_FAST_STARTUP $=1$

VES3D_PLATFORM=stampede

FFTW_DIR=/opt/apps/intel18/impi18_0/fftw3/3.3.8

FACEMAP_DIR=/home1/anonymous/USER/projects/boundary/ 」

$\hookrightarrow$ mobo-temp/face_map

QT_GRAPHICSSYSTEM_CHECKED $=1$

TACC_INTEL_BIN=/opt/intel/compilers_and_libraries_20 」 $\hookrightarrow$ 18.2.199/linux/bin/intel64

TACC_IMPI_INC=/opt/intel/compilers_and_libraries_201 」

$\hookrightarrow$ 8.2.199/linux/mpi/intel64/include

TACC_FFTW3_DIR=/opt/apps/intel18/impi18_0/fftw3/3.3.8 USER=USER

SLURM_NNODES $=1$

IDEV_QDEL=scancel

__LMOD_REF_COUNT_QT_QPA_PLATFORM_PLUGIN_PATH=/opt/ap 」

$\hookrightarrow$ ps/qt5/5.11.2/plugins: 1 
LS_COLORS $=r s=0: \mathrm{di}=38 ; 5 ; 27: \ln =38 ; 5 ; 51: \mathrm{mh}=44 ; 38 ; 5 ; 15: \mathrm{p}_{\mathrm{J}}$ $\mathrm{i}=40 ; 38 ; 5 ; 11: \mathrm{so}=38 ; 5 ; 13: \mathrm{do}=38 ; 5 ; 5: \mathrm{bd}=48 ; 5 ; 232 ; 38$ 」

$\rightarrow \quad ; 5 ; 11: \mathrm{cd}=48 ; 5 ; 232 ; 38 ; 5 ; 3:$ or $=48 ; 5 ; 232 ; 38 ; 5 ; 9: \mathrm{mi}=0$ 」

$\hookrightarrow \quad 5 ; 48 ; 5 ; 232 ; 38 ; 5 ; 15: \mathrm{su}=48 ; 5 ; 196 ; 38 ; 5 ; 15: \mathrm{sg}=48 ; 5 ; 1$ 」

$\rightarrow 1 ; 38 ; 5 ; 16: \mathrm{ca}=48 ; 5 ; 196 ; 38 ; 5 ; 226: \mathrm{tw}=48 ; 5 ; 10 ; 38 ; 5 ; 1$ 」

$\rightarrow \quad 6: \mathrm{ow}=48 ; 5 ; 10 ; 38 ; 5 ; 21: \mathrm{st}=48 ; 5 ; 21 ; 38 ; 5 ; 15: \mathrm{ex}=38 ; 5 ;$ 」

$\hookrightarrow \quad 34: * . \operatorname{tar}=38 ; 5 ; 9: * . \operatorname{tgz}=38 ; 5 ; 9: * . \operatorname{arc}=38 ; 5 ; 9: * . \operatorname{arj}=$ 」

$\hookrightarrow \quad 38 ; 5 ; 9: * . \operatorname{taz}=38 ; 5 ; 9: * .1 \mathrm{ha}=38 ; 5 ; 9: * .1 \mathrm{z} 4=38 ; 5 ; 9: *$.

$\hookrightarrow \quad \mathrm{zh}=38 ; 5 ; 9: * .1 \mathrm{zma}=38 ; 5 ; 9: * . \mathrm{tlz}=38 ; 5 ; 9: * . \mathrm{txz}=38 ; 5$ 」

$\hookrightarrow \quad ; 9: * . t z o=38 ; 5 ; 9: * . t 7 z=38 ; 5 ; 9: * . z i p=38 ; 5 ; 9: * . z=38$ 」

$\hookrightarrow \quad ; 5 ; 9: * . z=38 ; 5 ; 9: * . d z=38 ; 5 ; 9: * . g z=38 ; 5 ; 9: * .1 r z=38$ 」

$\hookrightarrow \quad ; 5 ; 9: * .1 z=38 ; 5 ; 9: * .1 z 0=38 ; 5 ; 9: * . x z=38 ; 5 ; 9: * . b z 2=$ 」

$\hookrightarrow \quad 38 ; 5 ; 9: * . b z=38 ; 5 ; 9: * . t b z=38 ; 5 ; 9: * . t b z 2=38 ; 5 ; 9: * . 」$

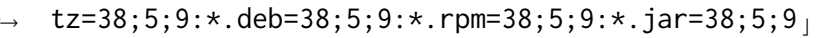

$\hookrightarrow \quad: *$.war $=38 ; 5 ; 9: *$.ear $=38 ; 5 ; 9: *$. sar $=38 ; 5 ; 9: *$. rar $=38$ ।

$\hookrightarrow \quad ; 5 ; 9: * . a l z=38 ; 5 ; 9: *$. ace $=38 ; 5 ; 9: * . z 00=38 ; 5 ; 9: * . c p$ 」

$\hookrightarrow \quad$ io $=38 ; 5 ; 9: * .7 z=38 ; 5 ; 9: * . r z=38 ; 5 ; 9: * . c a b=38 ; 5 ; 9: *$ 」

$\hookrightarrow \quad$.jpg $=38 ; 5 ; 13: *$.jpeg $=38 ; 5 ; 13: *$.gif $=38 ; 5 ; 13: * . \mathrm{bmp}=$ 」

$\hookrightarrow \quad 38 ; 5 ; 13: * . p b m=38 ; 5 ; 13: * . p g m=38 ; 5 ; 13: * . p p m=38 ; 5 ; 1$ 」

$\hookrightarrow \quad 3: *$. tga $=38 ; 5 ; 13: * . x b m=38 ; 5 ; 13: * . x p m=38 ; 5 ; 13: * . t i$

$\rightarrow \mathrm{f}=38 ; 5 ; 13: *$.tiff $=38 ; 5 ; 13: *$.png $=38 ; 5 ; 13: * . \mathrm{svg}=38$; 」

$\hookrightarrow \quad 5 ; 13: *$. svgz $=38 ; 5 ; 13: *$. mng $=38 ; 5 ; 13: * . p c x=38 ; 5 ; 13:$ 」

$\hookrightarrow \quad$ *.mov $=38 ; 5 ; 13: * . \mathrm{mpg}=38 ; 5 ; 13: *$.mpeg $=38 ; 5 ; 13: * . \mathrm{m} 2 \mathrm{v}$

$\hookrightarrow \quad=38 ; 5 ; 13: * . m k v=38 ; 5 ; 13: *$. webm $=38 ; 5 ; 13: *$. ogm $=38 ; 5$ 」

$\rightarrow \quad ; 13: * . m p 4=38 ; 5 ; 13: * . m 4 v=38 ; 5 ; 13: * . m p 4 v=38 ; 5 ; 13: *$ 」

$\hookrightarrow \quad$.vob $=38 ; 5 ; 13: * . q t=38 ; 5 ; 13: *$.nuv $=38 ; 5 ; 13: *$. wmv $=38$ 」

$\hookrightarrow \quad ; 5 ; 13: *$.asf $=38 ; 5 ; 13: * . r m=38 ; 5 ; 13: * . r m v b=38 ; 5 ; 13:$

$\hookrightarrow \quad * . \mathrm{flc}=38 ; 5 ; 13: *$. avi $=38 ; 5 ; 13: * . \mathrm{fli}=38 ; 5 ; 13: * . \mathrm{flv}=$ 」

$\hookrightarrow \quad 38 ; 5 ; 13: * . g l=38 ; 5 ; 13: * . d l=38 ; 5 ; 13: * . x c f=38 ; 5 ; 13:$,

$\hookrightarrow \quad$ *. xwd $=38 ; 5 ; 13: *$.yuv $=38 ; 5 ; 13: *$. cgm $=38 ; 5 ; 13: *$. emf $=$ 」

$\hookrightarrow \quad 38 ; 5 ; 13: *$.axv $=38 ; 5 ; 13: *$. an $x=38 ; 5 ; 13: *$. ogv $=38 ; 5 ; 1$ 」

$\hookrightarrow 3: *$. ogx $=38 ; 5 ; 13: *$. aac $=38 ; 5 ; 45: * . a u=38 ; 5 ; 45: * . f l a$ ।

$\hookrightarrow \mathrm{c}=38 ; 5 ; 45: *$. mid $=38 ; 5 ; 45: *$. midi $=38 ; 5 ; 45: *$. mka $=38$; 」

$\hookrightarrow \quad 5 ; 45: *$.mp $3=38 ; 5 ; 45: * . \mathrm{mpc}=38 ; 5 ; 45: *$. ogg $=38 ; 5 ; 45: *$ 」

$\hookrightarrow \quad . r a=38 ; 5 ; 45: *$. wav $=38 ; 5 ; 45: *$. axa $=38 ; 5 ; 45: *$.oga $=38$ 」

$\hookrightarrow \quad ; 5 ; 45: * . s p x=38 ; 5 ; 45: * . x s p f=38 ; 5 ; 45:$
LD_LIBRARY_PATH=/home1/apps/intel18/impi18_0/p4est/2 」 $\hookrightarrow \quad .0 / 1 \mathrm{ib}: /$ home1/apps/intel18/impi18_0/vtk/8.1.1/li

$\hookrightarrow$ b:/opt/apps/gcc/6.3.0/lib64:/opt/apps/gcc/6.3.0/।

$\hookrightarrow \quad \mathrm{ib}: / o p t / a p p s / q t 5 / 5.11 .2 / \mathrm{ib}: / o p t / a p p s / i n t e l 18 / \mathrm{b}$ 」

$\hookrightarrow$ oost/1.68/lib:/home1/apps/intel18/impi18_0/petsc 」

$\hookrightarrow$ /3.10/skylake-i64/lib:/opt/apps/libfabric/1.7.0/।

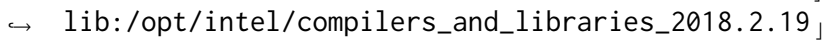

$\hookrightarrow$ 9/linux/mpi/intel64/lib:/opt/intel/debugger_2018」

$\hookrightarrow$ /libipt/intel64/lib:/opt/intel/debugger_2018/iga

$\hookrightarrow /$ ib:/opt/intel/compilers_and_libraries_2018.2.1」

$\hookrightarrow$ 99/linux/daal/../tbb/lib/intel64_lin/gcc4.4:/opt」

$\hookrightarrow$ /intel/compilers_and_libraries_2018.2.199/linux/」

$\hookrightarrow$ daal/lib/intel64_lin:/opt/intel/compilers_and_li

$\hookrightarrow$ braries_2018.2.199/linux/tbb/lib/intel64/gcc4.7: 」

$\hookrightarrow$ /opt/intel/compilers_and_libraries_2018.2.199/li 」

$\hookrightarrow$ nux/mkl/lib/intel64_lin:/opt/intel/compilers_and

$\hookrightarrow$ _libraries_2018.2.199/1inux/compiler/lib/intel64」

$\hookrightarrow$ _lin:/opt/intel/compilers_and_libraries_2018.2.1」

$\hookrightarrow$ 99/linux/ipp/lib/intel64:/opt/intel/compilers_an」

$\hookrightarrow$ d_libraries_2018.2.199/linux/compiler/lib/intel6」

$\hookrightarrow$ 4:/opt/apps/intel18/impi18_0/fftw3/3.3.8/1ib:/ho」

$\hookrightarrow$ me1/anonymous/USER/installs/cgal/lib64:/home1/an」

$\hookrightarrow$ onymous/USER/installs/gmp/lib:/home1/anonymous/U

$\hookrightarrow$ SER/installs/mpfr/lib:/home1/anonymous/USER/proj 」

$\hookrightarrow$ ects/boundary/contact3d/lib

TACC_QT5_DIR=/opt/apps/qt5/5.11.2

PSTLROOT=/opt/intel/compilers_and_libraries_2018.2.1 」

$\hookrightarrow$ 99/linux/pstl

TACC_PETSC_DIR=/home1/apps/intel18/impi18_0/petsc/3. 」

$\hookrightarrow \quad 10 /$

TRACKER__ $=1$

XALT_DATE_TIME=2019_05_20_15_39_13_1805

TACC_NODE_TYPE $=s k X$

SLURM_TACC_NODES $=1$

SLURM_JOBID $=3614475$

PERSONAL_PATH__ $=1$

CPATH=/opt/intel/compilers_and_libraries_2018.2.199/

$\hookrightarrow \quad$ linux/pstl/include:/opt/intel/compilers_and_libr 」

$\hookrightarrow$ aries_2018.2.199/linux/daal/include:/opt/intel/c 」

$\hookrightarrow$ ompilers_and_libraries_2018.2.199/linux/tbb/incl 」

$\hookrightarrow$ ude:/opt/intel/compilers_and_libraries_2018.2.19」

$\hookrightarrow$ 9/linux/mkl/include:/opt/intel/compilers_and_lib」

$\hookrightarrow$ raries_2018.2.199/linux/ipp/include

IFC_BIN=/opt/intel/compilers_and_libraries_2018.2.19 」

$\hookrightarrow$ 9/linux/bin/intel64

TACC_MKL_LIB=/opt/intel/compilers_and_libraries_2018」

$\hookrightarrow \quad .2 .199 / 1$ inux/mkl/lib/intel64 
_ModuleTable004_=ImxvYWRPcmRlci JdPTkscHJvcFQ9e30sWyJ 」 $\hookrightarrow \quad$ zdGFja0RlcHRoIl09MCXbInN0YXR1cyJdPSJhY3RpdmUiLFs 」 $\hookrightarrow \quad$ idXNlck5hbWUiXT0icDRlc3QiLH0scGV0c2M9e1siZm4iXT0 $\hookrightarrow \quad$ iL29wdC9hcHBzL2ludGVsMTgvaW1waTE4XzAvbW9kdWxlZml」 $\hookrightarrow$ sZXMvcGV0c2MvMy4xMC1pNjQubHVhIixbImZ1bGxOYW11Ilo, $\hookrightarrow \quad$ IInBldHNjLzMuMTAtaTYOI ixbImxvYWRPcmRlci JdPTUscHJ 」 $\hookrightarrow \quad$ vcFQ9e30sWyJzdGFja0RlcHRoI109MCxbInN0YXR1cyJdPSJ 」 $\hookrightarrow$ hY3RpdmUiLFsidXNlck5hbWUiXT0icGV0c2MvMy4XMC1pNjQ 」 $\hookrightarrow \quad$ iLH0scXQ1PXtbImZuI109I i 9vcHQvYXBwcy9tb2R1bGVmaWx 」 $\hookrightarrow \quad$ lcy9xdDUvNS4xMS4yLmx1YSIsWyJmdWxsTmFtZSJdPSJxdDU」 $\hookrightarrow \quad$ VNS4xMS4yIixbImxvYWRPcmRlci JdPTcscHJvcFQ9e30s XALT_RUN_UUID=4b841ccf-cc37-40f7-9c17-e7bb2cb0fdad OLDSCRATCH=/oldscratch/anonymous/USER

SLURM_COMMAND=sbatch

_LLMOD_REF_COUNT__LMFILES_=/opt/apps/modulefiles/int । $\hookrightarrow$ el/18.0.2.lua:1;/opt/apps/modulefiles/libfabric/」

$\hookrightarrow$ 1.7.0.lua:1;/opt/apps/intel18/modulefiles/impi/1」

$\hookrightarrow$ 8.0.2.lua:1;/opt/apps/intel18/impi18_0/modulefil」

$\hookrightarrow$ es/fftw3/3.3.8.lua:1;/opt/apps/intel18/impi18_0/।

$\hookrightarrow$ modulefiles/petsc/3.10-i64.1ua: 1 ;/opt/apps/intel」

$\hookrightarrow$ 18/modulefiles/boost/1.68.lua: 1 ;/opt/apps/module

$\hookrightarrow$ files/qt5/5.11.2.lua:1;/opt/apps/intel18/impi18_」

$\hookrightarrow \quad$ /modulefiles/vtk/8.1.1.lua:1;/opt/apps/intel18/」

$\hookrightarrow \quad$ impi18_0/modulefiles/p4est/2.0.lua: 1

TACC_BOOST_DIR=/opt/apps/intel18/boost $/ 1.68$

PVFMM_INC=/home1/anonymous/USER/installs/pvfmm/inclu 」 $\hookrightarrow$ de/pvfmm

SLURM_NTASKS $=1$

PETSC_ROOT=/home1/apps/intel18/impi18_0/petsc/3.10/

SLURM_TACC_JOBNAME $=i d v 31559$

LMOD_FAMILY_MPI_VERSION $=18.0 .2$

TACC_PETSC_LIB=/home1/apps/intel18/impi18_0/petsc/3.」 $\hookrightarrow$ 10/skylake-i64/lib

ARCHIVER=ranch. tacc . utexas . edu

NLSPATH=/opt/intel/debugger_2018/gdb/inte164/share/1

$\hookrightarrow$ ocale/\%1_\%t/\%N:/opt/intel/compilers_and_librarie 」

$\hookrightarrow$ s_2018.2.199/linux/mkl/lib/intel64/locale/\%1_\%t/」

$\hookrightarrow \quad \% \mathrm{~N}$ : /opt/intel/compilers_and_libraries_2018.2.199」

$\hookrightarrow /$ linux/compiler/lib/intel64/locale/\%1_\%t/\%N

__LMOD_REF_COUNT_I_MPI_ROOT=/opt/intel/compilers_and 」

$\hookrightarrow$ _libraries_2018.2.199/linux/mpi:1

LMOD_FAMILY_QT_VERSION=5.11.2

TACC_QT5_BIN=/opt/apps/qt5/5.11.2/bin

$\mathrm{PATH}=/ \mathrm{home} 1 / \mathrm{apps} /$ intel18/impi18_0/p4est/2.0/bin:/hom

$\hookrightarrow$ e1/apps/intel18/impi18_0/vtk/8.1.1/bin:/opt/apps」

$\hookrightarrow$ /gcc/6.3.0/bin:/opt/apps/qt5/5.11.2/bin:/opt/app」

$\hookrightarrow$ s/intel18/boost/1.68/bin:/home1/apps/intel18/imp」

$\hookrightarrow \quad$ i18_0/petsc/3.10/skylake-i64/bin:/opt/apps/libfa 」

$\hookrightarrow$ bric/1.7.0/bin:/opt/apps/intel18/impi/18.0.2/bin」

$\hookrightarrow \quad$ :/opt/intel/compilers_and_libraries_2018.2.199/1」

$\hookrightarrow \quad$ inux/mpi/intel64/bin:/opt/intel/compilers_and_li 」

$\hookrightarrow$ braries_2018.2.199/linux/bin/intel64:/usr/lib64/」

$\hookrightarrow$ qt-3.3/bin:/usr/local/bin:/bin:/usr/bin:/opt/del」

$\hookrightarrow \quad$ l/srvadmin/bin:/opt/apps/intel18/impi18_0/fftw3/」

$\hookrightarrow \quad 3.3 .8 / \mathrm{bin}$

MAIL=/var/spool/mail/USER
TACC_SYSTEM=stampede 2

_ModuleTable001_=X01 vZHVsZVRhYmxIXz17WyJNVHZlcnNpb24」

$\hookrightarrow \quad$ iXTOzLFsiY19yZWJ1aWxkVGItZSJdPWZhbHN1LFsiY19zaG9」

$\hookrightarrow \quad$ ydFRpbWUiXT1mYWxzZSxkZXB0aFQ9e30sZmFtaWx5PXtbIk1 」

$\hookrightarrow$ QSSJdPSJpbXBpIixbImNvbXBpbGVyIl09ImludGVsIixbInF 」

$\hookrightarrow$ OII09InFONSIsWyJ2dGsiXT0idnRrIix9LG1UPXtib29zdD1 」

$\hookrightarrow \quad$ 7Wy Jmbi JdPSIvb3B0L2FwcHMvaW50ZWwxOC9tb2R1bGVmaWx

$\hookrightarrow \quad$ lcy9ib29zdC8xLjY4Lmx1YSIsWyJmdWxsTmFtZSJdPSJib29

$\hookrightarrow \quad z d C 8 x L j Y 4 I i x b I m x v Y W R P c m R l c i J d P T Y s c H J v c F Q 9 e 30$ sWyJ

$\hookrightarrow \quad$ zdGFja0RlcHRoIl09MCxbInN0YXR1cyJdPSJhY3RpdmUiLFs 」

$\hookrightarrow \quad$ idXNlck5hbWUiXT0iYm9vc3QiLH0sZmZOdzM9e1siZm4iXT0」

$\hookrightarrow \quad$ iL29wdC9hcHBzL2ludGVsMTgvaW1waTE4XzAvbW9kdWxl

SLURM_TASKS_PER_NODE $=1$

ICC_BIN=/opt/intel/compilers_and_libraries_2018.2.19 」

$\hookrightarrow$ 9/linux/bin/intel64

__LMOD_REF_COUNT_NLSPATH=/opt/intel/debugger_2018/gd 」

$\hookrightarrow$ b/intel64/share/locale/\%1_\%t/\%N:1;/opt/intel/com」

$\hookrightarrow$ pilers_and_libraries_2018.2.199/linux/mkl/lib/in」

$\hookrightarrow$ tel64/locale/\%1_\%t/\%N:1;/opt/intel/compilers_and

$\hookrightarrow$ _libraries_2018.2.199/linux/compiler/lib/intel64」

$\hookrightarrow /$ locale/\%1_\%t/\% : 1

I_MPI_OFI_LIBRARY=/opt/apps/libfabric/1.7.0/lib/libf 」

$\hookrightarrow$ abric.so

STOCKYARD=/work/anonymous/USER

RUNNING_IDEV $=1$

SLURM_WORKING_CLUSTER=stampede2:206.76.192.2:6820:84 」

$\hookrightarrow \quad 48$

GEOGRAM_DIR=/home1/anonymous/USER/projects/boundary/ 」

$\hookrightarrow$ mobo-temp/geogram_1.6.3

_ $=/ \mathrm{bin} / \mathrm{env}$

WORK=/work/anonymous/USER/stampede 2

TACCINFO=/usr $/$ local/etc $/$ taccinfo

SLURM_JOB_ID $=3614475$

TACC_VTK_LIB=/home1/apps/intel18/impi18_0/vtk/8.1.1/ 」 $\hookrightarrow \quad$ lib

OLDWORK=/work/anonymous/USER

TBBROOT=/opt/intel/compilers_and_libraries_2018.2.19」

$\hookrightarrow \quad 9 / 1$ inux $/$ tbb

TACC_LIBFABRIC_LIB=/opt/apps/libfabric/1.7.0/1 ib

$\mathrm{PWD}=/$ home $1 /$ anonymous/USER

INPUTRC=/etc/inputrc

SLURM_JOB_USER=USER

SLURM_QUEUE $=$ skx-dev

SLURM_TACC_NCORES_SET $=1$

_LMFILES_=/opt/apps/modulefiles/intel/18.0.2.lua:/op 」

$\hookrightarrow$ t/apps/modulefiles/libfabric/1.7.0.lua:/opt/apps 」

$\hookrightarrow /$ intel18/modulefiles/impi/18.0.2.lua:/opt/apps/i 」

$\hookrightarrow$ ntel18/impi18_0/modulefiles/fftw3/3.3.8.lua:/opt 」

$\hookrightarrow$ /apps/intel18/impi18_0/modulefiles/petsc/3.10-i6」

$\hookrightarrow$ 4.lua:/opt/apps/intel18/modulefiles/boost/1.68.1」

$\hookrightarrow$ ua:/opt/apps/modulefiles/qt5/5.11.2.lua:/opt/app」

$\hookrightarrow$ s/intel18/impi18_0/modulefiles/vtk/8.1.1.lua:/op」

$\hookrightarrow$ t/apps/intel18/impi18_0/modulefiles/p4est/2.0.lua

TACC_P4EST_DIR=/home1/apps/intel18/impi18_0/p4est/2.0 LANG=en_US.UTF-8 
__LMOD_REF_COUNT_PYTHONPATH=/home1/apps/intel18/impi 」 $\hookrightarrow$ 18_0/vtk/8.1.1/lib/site-packages/mpi4py: 1 ;/home 1 」

$\hookrightarrow$ /apps/intel18/impi18_0/vtk/8.1.1/1ib/python2.7/s」

$\hookrightarrow \quad$ ite-packages/vtk: 1

HEDGEHOG_CC=mpicc

VTK_INC_DIR=/include/vtk-8.1/

MODULEPATH=/opt/apps/qt5.11.2/modulefiles:/opt/apps/।

$\hookrightarrow$ intel18/impi18_0/modulefiles:/opt/apps/intel18/m」

$\hookrightarrow$ odulefiles:/opt/apps/xsede/modulefiles:/opt/apps」

$\hookrightarrow$ /modulefiles:/opt/modulefiles

_ModuleTable_Sz_=6

SLURM_JOB_UID $=856401$

LOADEDMODULES=intel/18.0.2: libfabric/1.7.0: impi/18.0, $\hookrightarrow \quad .2: \mathrm{fftw} 3 / 3.3 .8:$ petsc/3.10-i64:boost/1.68: qt5/5.1」 $\hookrightarrow \quad 1.2: \mathrm{vtk} / 8.1 .1: \mathrm{p} 4 \mathrm{est} / 2.0$

SLURM_NODEID $=0$

idev_has_user_PERL5LIB=no

_BASHRC_SOURCED__ $=1$

SLURM_TACC_ACCOUNT=TG-DPP1 30002

TACC_VTK_DIR=/home1/apps/intel18/impi18_0/vtk/8.1.1

SLURM_SUBMIT_DIR=/home1/anonymous/USER

TACC_VEC_FLAGS $=-x$ CORE-AVX2 -axCORE-AVX512,MIC-AVX512

I_MPI_F90 $=$ ifort

LMOD_CMD=/opt/apps/1mod/1mod/libexec/lmod

SLURM_TASK_PID $=258435$

SLURM_NPROCS $=1$

I_MPI_CC $=$ icC

_ModuleTable005_=WyJzdGFja0RlcHRoI109MCXbInN0YXR1 cyJ 」

$\hookrightarrow$ dPSJhY3RpdmUiLFsidXNlck5hbWUiXT0icXQ1 Iix9LHZOaz1」

$\hookrightarrow \quad 7$ WyJmbi JdPSIvb3B0L2FwcHMvaW50ZWwxOC9pbXBpMThfMC9」

$\hookrightarrow$ tb2R1bGVmaWxlcy92dGsv0C4xLjEubHVhIixbImZ1bGxOYW1」

$\hookrightarrow \quad$ IIl09InZ0ay84LjEuMSIsWyJsb2FkT3JkZXIiXT04LHByb3B 」

$\hookrightarrow \quad$ UPXt9LFsic3RhY2tEZXB0aCJdPTAsWyJzdGFOdXMiXT0iYWN

$\hookrightarrow$ 0aXZ1IixbInVzZXJOYW11I109InZ0ayIsfSX9LG1wYXRoQT1」

$\hookrightarrow$ 7Ii9vcHQvYXBwcy9xdDUuMTEuMi9tb2R1bGVmaWxlcyIsIi9」

$\hookrightarrow \quad$ vcHQvYXBwcy9pbnRIbDE4L21tcGkxOF8wL21vZHVsZWZpbGV」

$\hookrightarrow \quad$ zIiwiL29wdC9hcHBzL2ludGVsMTgvbW9kdWxlZmlsZXMiLCI」

$\hookrightarrow \quad$ vb3B0L2FwcHMveHN1ZGUvbW9kdWxlZmlsZXMiLCIvb3B0

MOBO_DIR=/home1/anonymous/USER/projects/boundary/mob 」

$\hookrightarrow \quad$ o-temp

SLURM_CPUS_ON_NODE $=96$

DAALROOT=/opt/intel/compilers_and_libraries_2018.2.1」

$\hookrightarrow$ 99/linux/daal

TACC_P4EST_LIB=/home1/apps/intel18/impi18_0/p4est/2. 」

$\hookrightarrow \quad 0 / 1 \mathrm{ib}$

SSH_ASKPASS=/usr/libexec/openssh/gnome-ssh-askpass

SLURM_PROCID $=0$

ENVIRONMENT $=$ BATCH

TACC_MKL_INC=/opt/intel/compilers_and_libraries_2018」

$\hookrightarrow .2 .199 / \mathrm{linux} / \mathrm{mkl} / \mathrm{include}$

SLURM_JOB_NODELIST $=$ c506-033

HOME=/home1/anonymous/USER

SHLVL $=4$

FI_PROVIDER $=$ psm2

TACC_DOMAIN=stampede2

SLURM_LOCALID $=0$
_-_XALT_INITIAL_STATE__=LD_PRELOAD

__LMOD_REF_COUNT_PATH=/home1/apps/intel18/impi 18_0/p」

$\hookrightarrow$ 4est/2.0/bin:1;/home1/apps/intel18/impi18_0/vtk/

$\hookrightarrow \quad 8.1 .1 / \mathrm{bin}: 1$;/opt/apps/gcc/6.3.0/bin:2;/opt/apps/」

$\hookrightarrow$ qt5/5.11.2/bin:1;/opt/apps/intel18/boost/1.68/bi

$\hookrightarrow \mathrm{n}: 1 ; /$ home1/apps/intel18/impi18_0/petsc/3.10/skyl」

$\hookrightarrow$ ake-i64/bin:1;/opt/apps/libfabric/1.7.0/bin:1;/o 」

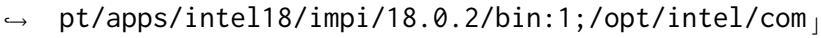

$\hookrightarrow$ pilers_and_libraries_2018.2.199/linux/mpi/intel6」

$\hookrightarrow$ 4/bin:1;/opt/intel/compilers_and_libraries_2018.」

$\hookrightarrow$ 2.199/linux/bin/intel64:1;/usr/lib64/qt-3.3/bin:

$\hookrightarrow$ 1;/usr/local/bin:1;/bin:1;/usr/bin:1;/opt/dell/s 」

$\hookrightarrow$ rvadmin/bin:1;/opt/apps/intel18/impi18_0/fftw3/3」

$\hookrightarrow \quad$.3.8/bin:1

TACC_FAMILY_COMPILER=intel

TACC_INTEL_LIB=/opt/intel/compilers_and_libraries_20 」

$\hookrightarrow$ 18.2.199/linux/compiler/lib/intel64

I_MPI_CXX=icpc

TACC_PETSC_INC=/home1/apps/intel18/impi18_0/petsc/3. 」

$\hookrightarrow$ 10/skylake-i64/include

TACC_FAMILY_VTK=vtk

__LMOD_REF_COUNT_CPATH=/opt/intel/compilers_and_libr 」

$\hookrightarrow$ aries_2018.2.199/linux/pstl/include:1;/opt/intel」

$\hookrightarrow$ /compilers_and_libraries_2018.2.199/linux/daal/i 」

$\hookrightarrow$ nclude:1;/opt/intel/compilers_and_libraries_2018」

$\hookrightarrow$.2.199/linux/tbb/include:1;/opt/intel/compilers_」

$\hookrightarrow$ and_libraries_2018.2.199/linux/mkl/include:1;/op」

$\hookrightarrow \quad$ t/intel/compilers_and_libraries_2018.2.199/linux 」

$\hookrightarrow \quad /$ ipp/include: 1

TACC_BOOST_LIB=/opt/apps/intel18/boost/1.68/1ib

TACC_P4EST_INC=/home1/apps/intel18/impi18_0/p4est/2. 」

$\hookrightarrow \quad 0 /$ include

_ModuleTable002_=ZmlsZXMvZmZ0dzMvMy4zLjgubHVhIixbImZ」

$\hookrightarrow$ 1bGx0YW11Il09ImZmdHczLzMuMy44I ixbImxvYWRPcmRlci J

$\hookrightarrow$ dPTQscHJvcFQ9e30sWyJzdGFja0RlcHRoIl09MCxbInN0YXR 」

$\hookrightarrow \quad$ 1cyJdPSJhY3RpdmUiLFsidXN1ck5hbWUiXT0iZmZ0dzMiLH0,

$\hookrightarrow$ saW1waT17WyJmbi JdPSIvb3B0L2FwcHMvaW50ZWwXOC9tb2R 」

$\hookrightarrow$ 1bGVmaWxlcy9pbXBpLzE4LjAuMi5sdWEiLFsiZnVsbE5hbWU

$\hookrightarrow \quad$ iXT0iaW1waS8x0C4wLjIiLFsibG9hZE9yZGVyIl09Myxwcm9 」

$\hookrightarrow \quad$ wVD17fSxbInNOYWNrRGVwdGgiXTOWLFsic3RhdHVzI109ImF 」

$\hookrightarrow$ jdG12ZSIsWyJ1c2VyTmFtZSJdPSJpbXBpI ix9LGludGVsPXt

$\hookrightarrow$ bImZuI109Ii9vcHQvYXBwcy9tb2R1bGVmaWxlcy9pbnRlbC8 」

$\hookrightarrow \quad$ xOC4wLjIubHVhIixbImZ1bGxOYW11I109ImludGVsLzE4

SLURM_CLUSTER_NAME=stampede 2

SLURM_JOB_CPUS_PER_NODE $=96$

SLURM_JOB_GID $=814474$

IFC_LIB=/opt/intel/compilers_and_libraries_2018.2.19 」

$\hookrightarrow$ 9/linux/compiler/lib/intel64

SLURM_GTIDS $=0$

SLURM_SUBMIT_HOST=login2. stampede2. tacc.utexas.edu __LMOD_REF_COUNT_INCLUDE=/home1/apps/intel18/impi 18_ 」

$\hookrightarrow \quad 0 / v t k / 8.1 .1 /$ include: 1 ;/opt/apps/gcc/6.3.0/includ $\hookrightarrow \mathrm{e}: 1$

BLENDSURF_DIR=/home1/anonymous/USER/projects/boundar 」 $\hookrightarrow \mathrm{y} / \mathrm{mobo}$-temp/blendsurf3

BASH_ENV=/etc/tacc/tacc_functions 
idev_ip $=$ c506-033

SLURM_JOB_PARTITION=skx-dev

I_MPI_FC=ifort

TACC_VTK_INC=/home1/apps/intel18/impi18_0/vtk/8.1.1/ 」

$\hookrightarrow$ include

PVFMM_LIB=/home1/anonymous/USER/installs/pvfmm/lib/p 」

$\hookrightarrow \quad \mathrm{Vfmm}$

LOGNAME $=$ USER

ICC_LIB=/opt/intel/compilers_and_libraries_2018.2.19 」

$\hookrightarrow$ 9/linux/compiler/lib/intel64

TACC_FAMILY_MPI $=$ impi

LMOD_FAMILY_VTK=vtk

PYTHONPATH=/home1/apps/intel18/impi18_0/vtk/8.1.1/li 」

$\hookrightarrow$ b/site-packages/mpi4py:/home1/apps/intel18/impi1」

$\hookrightarrow$ 8_0/vtk/8.1.1/lib/python2.7/site-packages/vtk

CVS_RSH=ssh

QTLIB=/usr/lib64/qt-3.3/lib

LMOD_SETTARG_TITLE_BAR=yes

BOOST_ROOT=/opt/apps/intel18/boost/1.68

SSH_CONNECTION=206.76.192.52 60610 206.76.217.53 22

XDG_DATA_DIRS=/home1/anonymous/USER/. local/share/fla 」

$\hookrightarrow$ tpak/exports/share:/var/lib/flatpak/exports/shar 」

$\hookrightarrow$ e:/usr/local/share:/usr/share

LC_CTYPE=UTF-8

SLURM_JOB_ACCOUNT=TG-DPP130002

HEDGEHOG_CXX=mpicXX

MODULESHOME=/opt $/ \mathrm{apps} / 1 \mathrm{mod} / 1 \mathrm{mod}$

SLURM_JOB_NUM_NODES $=1$

_LLMOD_REF_COUNT_LIBRARY_PATH=/opt/intel/compilers_a 」

$\hookrightarrow$ nd_libraries_2018.2.199/linux/daal/../tbb/lib/in」

$\hookrightarrow$ tel64_lin/gcc4.4:1;/opt/intel/compilers_and_libr」

$\hookrightarrow$ aries_2018.2.199/linux/daal/lib/intel64_lin:1;/o」

$\hookrightarrow$ pt/intel/compilers_and_libraries_2018.2.199/linu

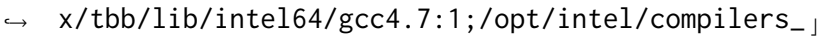

$\hookrightarrow$ and_libraries_2018.2.199/linux/mkl/lib/intel64_l」

$\hookrightarrow \quad$ in:1;/opt/intel/compilers_and_libraries_2018.2.1」

$\hookrightarrow$ 99/linux/compiler/lib/intel64_lin:1;/opt/intel/c 」

$\hookrightarrow$ ompilers_and_libraries_2018.2.199/linux/ipp/lib/ 」

$\hookrightarrow$ intel64:1

MPI_HOME=/opt/intel/compilers_and_libraries_2018.2.1」

$\hookrightarrow 99 /$ linux/mpi

LESSOPEN=||/usr/bin/lesspipe. sh \%s

LMOD_SETTARG_FULL_SUPPORT $=$ full

OMP_NUM_THREADS $=48$
__LMOD_REF_COUNT_LD_LIBRARY_PATH=/home1/apps/intel18 」 $\hookrightarrow$ /impi18_0/p4est/2.0/lib:1;/home1/apps/intel18/im」 $\hookrightarrow$ pi18_0/vtk/8.1.1/lib:1;/opt/apps/gcc/6.3.0/1ib64」

$\hookrightarrow \quad: 2 ;$ /opt/apps/gcc/6.3.0/lib:2;/opt/apps/qt5/5.11.」

$\hookrightarrow$ 2/lib:1;/opt/apps/intel18/boost/1.68/lib:1;/home 」

$\hookrightarrow$ 1/apps/intel18/impi18_0/petsc/3.10/skylake-i64/1」

$\hookrightarrow \quad \mathrm{ib}: 1$;/opt/apps/libfabric/1.7.0/lib:1;/opt/intel/」

$\hookrightarrow$ compilers_and_libraries_2018.2.199/linux/mpi/int 」

$\hookrightarrow$ el64/lib:1;/opt/intel/debugger_2018/libipt/intel」

$\hookrightarrow$ 64/lib:1;/opt/intel/debugger_2018/iga/lib:1;/opt 」

$\hookrightarrow$ /intel/compilers_and_libraries_2018.2.199/linux/」

$\hookrightarrow$ daal/../tbb/lib/intel64_lin/gcc4.4:1;/opt/intel/」

$\hookrightarrow$ compilers_and_libraries_2018.2.199/linux/daal/li 」

$\hookrightarrow$ b/intel64_lin:1;/opt/intel/compilers_and_librari 」

$\hookrightarrow$ es_2018.2.199/linux/tbb/lib/intel64/gcc4.7:1;/op 」

$\hookrightarrow$ t/intel/compilers_and_libraries_2018.2.199/linux 」

$\hookrightarrow$ /mkl/lib/intel64_lin:1;/opt/intel/compilers_and_」

$\hookrightarrow$ libraries_2018.2.199/1inux/compiler/lib/intel64_」

$\hookrightarrow \quad$ lin:2;/opt/intel/compilers_and_libraries_2018.2.」

$\hookrightarrow$ 199/linux/ipp/lib/intel64:1;/opt/intel/compilers 」

$\hookrightarrow$ _and_libraries_2018.2.199/linux/compiler/lib/int 」

$\hookrightarrow$ el64:1;/opt/apps/intel18/impi18_0/fftw3/3.3.8/li」

$\hookrightarrow \mathrm{b}: 1$

PKG_CONFIG_PATH=/opt/intel/compilers_and_libraries_2 」

$\hookrightarrow$ 018.2.199/linux/mkl/bin/pkgconfig:/opt/apps/inte 」

$\hookrightarrow$ 118/impi18_0/fftw3/3.3.8/lib/pkgconfig

HEDGEHOG_DIR=/home1/anonymous/USER/projects/boundary 」

$\hookrightarrow$ /mobo-temp

PROMPT_COMMAND $=\$\{$ X_SET_TITLE_BAR: $-:\}$

$\hookrightarrow \quad$ "\$USER $\$\{$ SHOST $\}: \$\{$ PWD $/$ \# $\$$ HOME $/ \sim\} "$

_Init_Default_Modules $=1$

TACC_FFTW3_LIB=/opt/apps/intel18/impi18_0/fftw3/3.3. 」 $\hookrightarrow \quad 8 / 1$ ib

LMOD_FAMILY_COMPILER=intel

TACC_IMPI_LIB=/opt/intel/compilers_and_libraries_201」

$\hookrightarrow$ 8.2.199/linux/mpi/intel64/lib

TACC_VTK_BIN=/home1/apps/intel18/impi18_0/vtk/8.1.1/ 」 $\hookrightarrow$ bin

VTK_LOCATION=/home1/apps/intel18/impi18_0/vtk/8.1.1

XDG_RUNTIME_DIR=/run/user $/ 856401$

ARCHIVE=/home/anonymous/USER

OLDHOME=/oldhome $1 /$ anonymous/USER

IDEV_PWD $=$ /home $1 /$ anonymous/USER

__LMOD_REF_COUNT_INTEL_LICENSE_FILE=/home1/anonymous 」

$\hookrightarrow$ /USER/intel/licenses:1;/opt/intel/licenses:1;/op 」

$\hookrightarrow \quad$ t/intel/compilers_and_libraries_2018.2.199/linux 」

$\hookrightarrow /$ licenses: 1

TACC_FAMILY_MPI_VERSION=18.0.2

__LMOD_REF_COUNT_PKG_CONFIG_PATH=/opt/intel/compiler 」

$\hookrightarrow$ S_and_libraries_2018.2.199/linux/mkl/bin/pkgconf 」

$\hookrightarrow \quad$ ig: 1 ;/opt/apps/intel18/impi18_0/fftw3/3.3.8/1ib/」

$\hookrightarrow$ pkgconfig: 1

TACC_QT5_LIB=/opt/apps/qt5/5.11.2/lib

LMOD_FAMILY_VTK_VERSION $=8.1 .1$

PVFMM_DIR=/home1/anonymous/USER/installs/pvfmm/share 」

$\hookrightarrow \quad / p v f m m$ 
Scalable Simulation of Realistic Volume Fraction Red Blood Cell Flows through Vascular Networks

LMOD_DIR=/opt/apps/lmod/1mod/libexec __LMOD_REF_COUNT_MANPATH=/opt/apps/libfabric/1.7.0/s 」

$\hookrightarrow$ hare/man:1;/opt/intel/compilers_and_libraries_20」

$\hookrightarrow$ 18.2.199/linux/mpi/man:1;/opt/intel/documentatio」

$\hookrightarrow$ n_2018/en/man/common:1;/opt/intel/documentation_」

$\hookrightarrow$ 2018/en/debugger/gdb-igfx/man: 1 ;/opt/intel/docum 」

$\hookrightarrow$ entation_2018/en/debugger/gdb-ia/man: 1 ; /opt/apps 」

$\hookrightarrow$ /intel18/impi18_0/fftw3/3.3.8/man: 1

INCLUDE=/home1/apps/intel18/impi18_o/vtk/8.1.1/inclu」 $\hookrightarrow$ de:/opt/apps/gcc/6.3.0/include

_ModuleTable006_=L2FwcHMvbW9kdWxlZmlsZXMiLCIvb3B0L 21 」

$\hookrightarrow \quad$ VZHVsZWZpbGVzIix9LFsic3lzdGVtQmFzZU1QQVRII109Ii9

$\hookrightarrow \quad$ vcHQvYXBwcy94c2VkZS9tb2R1bGVmaWxlczovb3B0L2FwcHM 」

$\hookrightarrow \quad$ vbW9kdWx1ZmlsZXM6L29wdC9tb2R1bGVmaWxlcyIsfQ==

PETSC_DIR=/home1/apps/intel18/impi18_o/petsc/3.10/

LMOD_FAMILY_QT=qt5

SCRATCH=/scratch/anonymous/USER

SLURM_TACC_NNODES_SET=1

SLURM_TACC_CORES $=1$

TACC_MKL_DIR=/opt/intel/compilers_and_libraries_2018」

$\hookrightarrow \quad$.2.199/linux/mkl

FI_PSM2_LAZY_CONN=1

LMOD_FAMILY_MPI=impi

TACC_MPI_GETMODE=impi_hydra

I_MPI_ROOT=/opt/intel/compilers_and_libraries_2018.2」

$\hookrightarrow .199 /$ linux/mpi

TACC_QT5_INC=/opt/apps/qt5/5.11.2/include

MACHINE_NAME=stampede

BASH_FUNC_sbatch()=() $\{$ echo -e "\nNOTIFICATION:

$\hookrightarrow$ sbatch not available on compute nodes. Use a login

$\hookrightarrow$ node. $\backslash n^{\prime \prime}$

\}

BASH_FUNC_module ()$=()\{$ if $[-z$

$\hookrightarrow \quad$ "\$\{LMOD_SH_DBG_ON+x $\} "]$; then

case "\$-" in

$\star v * x *$ )

__lmod_sh_dbg=' $v x^{\prime}$

; ;

$\star \mathrm{V} *$ )

_-lmod_sh_dbg= 'v'

$;$

$* x *$ )

__lmod_sh_dbg= ' $x$ '

; ;

esac;

$\mathrm{fi}$;

if $[-n$ "\$\{__lmod_sh_dbg:- $\}$ " $]$; then

set +\$__lmod_sh_dbg;

echo "Shell debugging temporarily silenced: export

$\hookrightarrow$ LMOD_SH_DBG_ON=1 for Lmod's output";

fi;

eval \$(\$LMOD_CMD bash "\$@") \&\& eval

$\hookrightarrow \$\left(\$\left\{L M O D \_S E T T A R G \_C M D:-:\right\}-s\right.$ sh $)$;

local_lmod_my_status $=\$$ ?;

if $[-\mathrm{n}$ "\$\{__lmod_sh_dbg:- $\}$ " ]; then

echo "Shell debugging restarted";

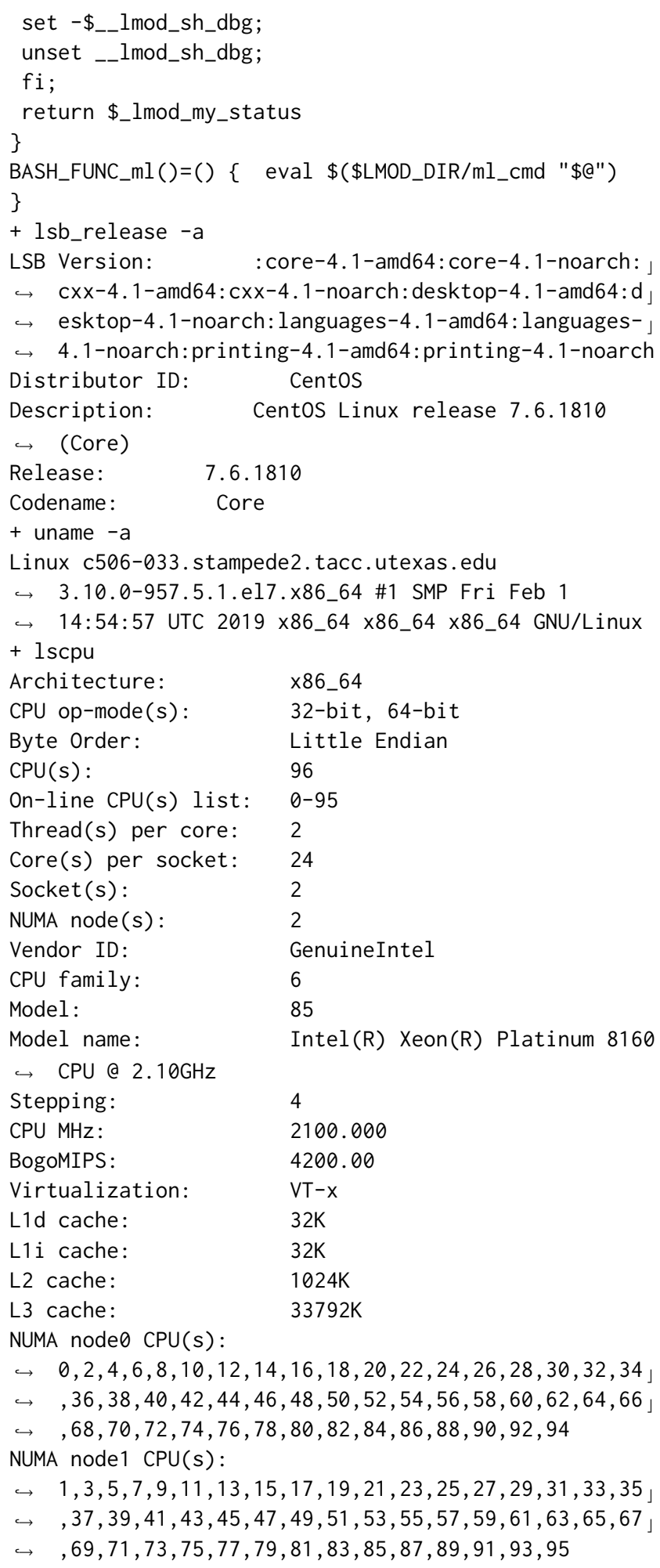


Flags:

fpu vme de pse tsc msr pae mce

$\hookrightarrow$ cx8 apic sep mtrr pge mca cmov pat pse36 clflush

$\hookrightarrow$ dts acpi mmx fxsr sse sse2 ss ht tm pbe syscall $\mathrm{nx}$

$\hookrightarrow$ pdpe1gb rdtscp Im constant_tsc art arch_perfmon

$\hookrightarrow$ pebs bts rep_good nopl xtopology nonstop_tsc

$\hookrightarrow$ aperfmperf eagerfpu pni pclmulqdq dtes64 monitor

$\hookrightarrow$ ds_cpl vmx smx est tm2 ssse3 sdbg fma cx16 xtpr

$\hookrightarrow$ pdcm pcid dca sse4_1 sse4_2 x2apic movbe popcnt

$\hookrightarrow \quad$ tsc_deadline_timer aes xsave avx f16c rdrand

$\hookrightarrow$ lahf_lm abm 3dnowprefetch epb cat_13 cdp_13

$\hookrightarrow \quad$ intel_ppin intel_pt ssbd mba ibrs ibpb stibp

$\hookrightarrow$ tpr_shadow vnmi flexpriority ept vpid fsgsbase

$\hookrightarrow$ tsc_adjust bmi1 hle avx2 smep bmi2 erms invpcid

$\hookrightarrow \quad$ rtm cqm mpx rdt_a avx512f avx512dq rdseed adx smap

$\hookrightarrow$ clflushopt clwb avx512cd avx512bw avx512vl

$\hookrightarrow \quad$ xsaveopt xsavec xgetbv1 cqm_llc cqm_occup_llc

$\hookrightarrow$ cqm_mbm_total cqm_mbm_local dtherm ida arat pln

$\hookrightarrow$ pts pku ospke spec_ctrl intel_stibp flush_l1d

+ cat /proc/meminfo

MemTotal: $\quad 196438176$ kB

MemFree: $\quad 190492500 \mathrm{kB}$

MemAvailable: 190025288 kB

Buffers: $\quad 0 \mathrm{kB}$

Cached: $\quad 138176 \mathrm{kB}$

SwapCached: $\quad 0 \mathrm{kB}$

Active: $\quad 158396 \mathrm{kB}$

Inactive: $\quad 111156 \mathrm{kB}$

Active(anon): $\quad 132300 \mathrm{kB}$

Inactive(anon): $\quad 84508 \mathrm{kB}$

Active(file): $\quad 26096 \mathrm{kB}$

Inactive(file): $26648 \mathrm{kB}$

Unevictable: $\quad 0 \mathrm{kB}$

Mlocked: $\quad 0 \mathrm{kB}$

SwapTotal: $\quad 0 \mathrm{kB}$

SwapFree: $\quad 0 \mathrm{kB}$

Dirty: $\quad 0 \mathrm{kB}$

Writeback: $\quad 0 \mathrm{kB}$

AnonPages: $\quad 132028 \mathrm{kB}$

Mapped: $\quad 27260 \mathrm{kB}$

Shmem: $\quad 84788 \mathrm{kB}$

Slab: $\quad 2495120 \mathrm{kB}$

SReclaimable: $\quad 580428 \mathrm{kB}$

SUnreclaim: $\quad 1914692$ kB

KernelStack: $25280 \mathrm{kB}$

PageTables: $7492 \mathrm{kB}$

NFS_Unstable: $\quad 0 \mathrm{kB}$

Bounce: $\quad 0 \mathrm{kB}$

WritebackTmp: $\quad 0 \mathrm{kB}$

CommitLimit: 182687500 kB

Committed_AS: $\quad 671048 \mathrm{kB}$

VmallocTotal: $34359738367 \mathrm{kB}$

VmallocUsed: $1826416 \mathrm{kB}$

VmallocChunk: 34257106468 kB

HardwareCorrupted: $\quad 0 \mathrm{kB}$

AnonHugePages: $53248 \mathrm{kB}$

CmaTotal: $0 \mathrm{kB}$

CmaFree:

\begin{tabular}{|c|c|c|c|c|}
\hline HugePages_Total: & 0 & & & \\
\hline HugePages_Free: & 0 & & & \\
\hline HugePages_Rsvd: & 0 & & & \\
\hline HugePages_Surp: & 0 & & & \\
\hline Hugepagesize: & 2048 & kB & & \\
\hline DirectMap4k: & 673600 & kB & & \\
\hline \multicolumn{5}{|l|}{ DirectMap2M: } \\
\hline \multicolumn{5}{|l|}{$\begin{array}{l}\text { DirectMap1G: } \\
+ \text { inxi-F -co }\end{array}$} \\
\hline \multicolumn{5}{|c|}{$\begin{array}{l}. / \text { collect_environment.sh: line 14: inxi: command not } \\
\hookrightarrow \text { found }\end{array}$} \\
\hline NAME & MAJ :MIN & SIZE & RO TYPE & MOUNTPOINT \\
\hline sda & $8: 0$ & $0223.6 \mathrm{G}$ & 0 disk & \\
\hline |-sda1 & $8: 1$ & $0 \quad 1 M$ & 0 part & \\
\hline |-sda2 & $8: 2$ & $0 \quad 1 G$ & 0 part & /boot \\
\hline$-s d a 3$ & $8: 3$ & $0222.6 \mathrm{G}$ & 0 part & \\
\hline |-rootvg01-lv01 & $253: 0$ & $0 \quad 75 G$ & $0 \mathrm{lvm}$ & / \\
\hline |-rootvg01-tmp & $253: 1$ & $0143.6 \mathrm{G}$ & $0 \mathrm{lvm}$ & /tmp \\
\hline '-rootvg01-var & $253: 2$ & $0 \quad 4 G$ & $0 \mathrm{lvm}$ & /var \\
\hline $\mathrm{sdb}$ & $8: 16$ & 0 & 0 disk & \\
\hline loop0 & $7: 0$ & 0 & 1 loop & \\
\hline loop1 & $7: 1$ & 0 & 1 loop & \\
\hline loop2 & $7: 2$ & 0 & 1 loop & \\
\hline loop3 & $7: 3$ & 0 & 1 loop & \\
\hline loop4 & $7: 4$ & 0 & 1 loop & \\
\hline loop5 & $7: 5$ & 0 & 1 loop & \\
\hline loop6 & $7: 6$ & 0 & 1 loop & \\
\hline loop7 & $7: 7$ & 0 & 1 loop & \\
\hline loop8 & $7: 8$ & 0 & 1 loop & \\
\hline loop9 & $7: 9$ & 0 & 1 loop & \\
\hline loop10 & $7: 10$ & 0 & 1 loop & \\
\hline loop11 & $7: 11$ & 0 & 1 loop & \\
\hline loop12 & $7: 12$ & 0 & 1 loop & \\
\hline loop13 & $7: 13$ & 0 & 1 loop & \\
\hline loop14 & $7: 14$ & 0 & 1 loop & \\
\hline loop15 & $7: 15$ & 0 & 1 loop & \\
\hline loop16 & $7: 16$ & 0 & 1 loop & \\
\hline loop17 & $7: 17$ & 0 & 1 loop & \\
\hline loop18 & $7: 18$ & 0 & 1 loop & \\
\hline loop19 & $7: 19$ & 0 & 1 loop & \\
\hline loop20 & $7: 20$ & 0 & 1 loop & \\
\hline loop21 & $7: 21$ & 0 & 1 loop & \\
\hline loop22 & $7: 22$ & 0 & 1 loop & \\
\hline loop23 & $7: 23$ & 0 & 1 loop & \\
\hline loop24 & $7: 24$ & 0 & 1 loop & \\
\hline loop25 & $7: 25$ & 0 & 1 loop & \\
\hline loop26 & $7: 26$ & 0 & 1 loop & \\
\hline loop27 & $7: 27$ & 0 & 1 loop & \\
\hline loop28 & $7: 28$ & 0 & 1 loop & \\
\hline loop29 & $7: 29$ & 0 & 1 loop & \\
\hline loop30 & $7: 30$ & 0 & 1 loop & \\
\hline loop31 & $7: 31$ & 0 & 1 loop & \\
\hline loop32 & $7: 32$ & 0 & 1 loop & \\
\hline loop33 & $7: 33$ & 0 & 1 loop & \\
\hline loop34 & $7: 34$ & 0 & 1 loop & \\
\hline
\end{tabular}


Scalable Simulation of Realistic Volume Fraction Red Blood Cell Flows through Vascular Networks

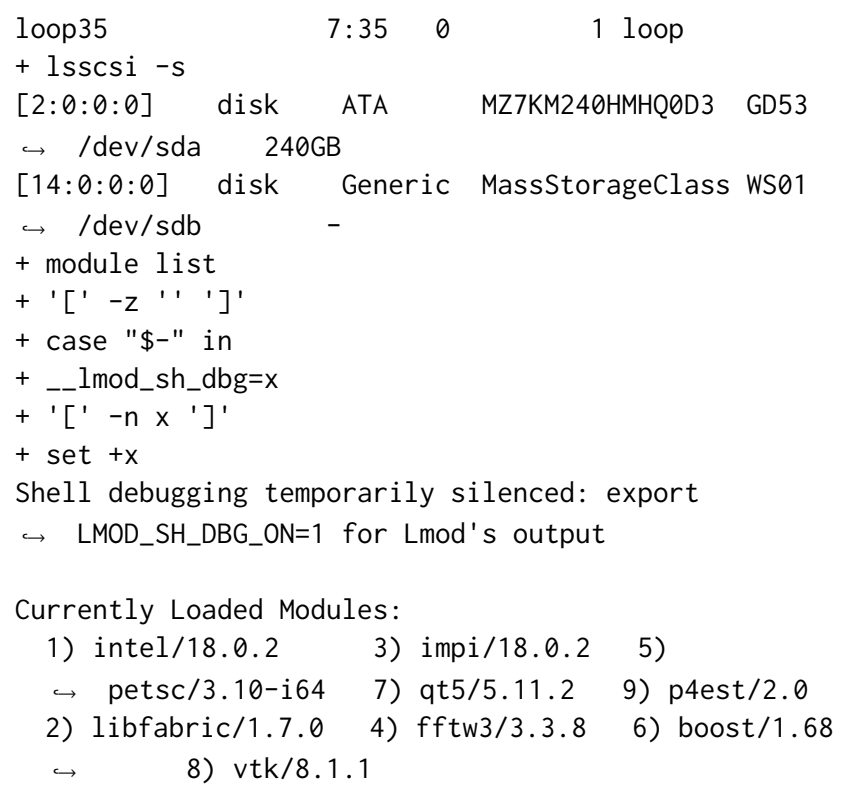

Shell debugging restarted

+ unset __lmod_sh_dbg

+ return 0

+ nvidia-smi

./collect_environment.sh: line 18: nvidia-smi:

$\hookrightarrow$ command not found

+ lshw -short -quiet -sanitize

+ cat

./collect_environment.sh: line 19: lshw: command not

$\hookrightarrow$ found

+1 spci

./collect_environment.sh: line 19: lspci: command not

$\hookrightarrow$ found

On Stampde2 $\mathrm{knl}$ nodes, the execution environment

$\hookrightarrow$ information is the following:

c455-061[knl](1020)\$./collect_environment.sh SLURM_NODELIST $=\mathrm{C} 455-061$

SLURM_CHECKPOINT_IMAGE_DIR=/var/slurm/checkpoint LMOD_FAMILY_COMPILER_VERSION $=18.0 .2$

MKLROOT=/opt/intel/compilers_and_libraries_2018.2.19」

$\hookrightarrow \quad$ 9/linux/mkl

I_MPI_STARTUP_MODE=pmi_shm_netmod

P4EST_DIR=/home1/apps/intel18/impi18_0/p4est/2.0

SLURM_JOB_NAME $=i d v 63880$

MANPATH=/opt/apps/libfabric/1.7.0/share/man:/opt/int 」

$\hookrightarrow$ el/compilers_and_libraries_2018.2.199/linux/mpi/」

$\hookrightarrow \mathrm{man}: /$ opt/intel/documentation_2018/en/man/common: 」

$\hookrightarrow$ /opt/intel/documentation_2018/en/debugger/gdb-ig」

$\hookrightarrow \mathrm{fx} / \mathrm{man}$ //opt/intel/documentation_2018/en/debugger 」

$\hookrightarrow$ /gdb-ia/man:/opt/apps/intel18/impi18_0/fftw3/3.3」

$\hookrightarrow .8 / \mathrm{man}$
TACC_IMPI_DIR=/opt/intel/compilers_and_libraries_201」 $\hookrightarrow$ 8.2.199/linux/mpi

TACC_FFTW3_INC=/opt/apps/intel18/impi18_0/fftw3/3.3.」 $\hookrightarrow$ 8/include

VTK_DIR=/home1/apps/intel18/impi18_0/vtk/8.1.1

XDG_SESSION_ID $=371971$

TACC_BOOST_INC=/opt/apps/intel18/boost/1.68/include

HOSTNAME $=c 455-061$

SLURMD_NODENAME $=c 455-061$

SLURM_TOPOLOGY_ADDR $=c 455-061$

TACC_INTEL_INC=/opt/intel/compilers_and_libraries_20 」

$\hookrightarrow$ 18.2.199/linux/compiler/include/intel64

_ModuleTable003_=LjAuMi IsWyJsb2FkT3JkZXIiXT0xLHByb3B

$\hookrightarrow \quad$ UPXt9LFsic3RhY2tEZXB0aCJdPTAsWyJzdGFOdXMiXT0iYWN

$\hookrightarrow$ 0aXZlIixbInVzZXJ0YW11Il09ImludGVsIix9LGxpYmZhYnJ

$\hookrightarrow \quad \mathrm{pYz17WyJmbiJdPSIvb3B0L2FwcHMvbW9kdW \times 1ZmlsZXMvbG1}$

$\hookrightarrow \quad i Z m F i c m l j L z E u N y 4 w L m x 1 Y S I s W y J m d W x s T m F t Z S J d P S J s a W J$ 」

$\hookrightarrow$ mYWJyaWMVMS43LjAiLFsibG9hZE9yZGVyIl09Mixwcm9wVD1」

$\hookrightarrow$ 7fSxbInJ1Z19jb3VudCJdPTEsWyJzdGFja0RlcHRoI109MSx ।

$\hookrightarrow$ bInNOYXR1cyJdPSJhY3RpdmUiLFsidXN1ck5hbWUiXT0ibGl」

$\hookrightarrow \quad i Z m F i c m l j I i x 9 L H A 0 Z X N 0 P X$ tbImZuIl09I i 9vcHQvYXBwcy9 」

$\hookrightarrow$ pbnRIbDE4L21tcGkXOF8wL21vZHVsZWZpbGVZL3A0ZXNOLzI

$\hookrightarrow \quad$ UMC5SdWEiLFsiZnVsbE5hbWUiXT0icDRlc3QvMi4wIixb

TACC_FAMILY_QT_VERSION $=5.11 .2$

SLURM_PRIO_PROCESS $=0$

SLURM_NODE_ALIASES $=($ null $)$

INTEL_LICENSE_FILE=/home1/anonymous/USER/intel/licen 」

$\hookrightarrow$ ses:/opt/intel/licenses:/opt/intel/compilers_and J

$\hookrightarrow$ _libraries_2018.2.199/linux/licenses

IPPROOT=/opt/intel/compilers_and_libraries_2018.2.19 」

$\hookrightarrow \quad 9 / 1$ inux/ipp

I_MPI_F77=ifort

MPICH_HOME=/opt/intel/compilers_and_libraries_2018.2 」 $\hookrightarrow \quad .199 /$ linux/mpi

TACC_PETSC_BIN=/home1/apps/intel18/impi18_0/petsc/3. 」

$\hookrightarrow 10 /$ skylake-i64/bin

SHELL=/bin/bash

TERM $=x$ term -256 color

__LMOD_REF_COUNT_MODULEPATH=/opt/apps/qt5.11.2/modul 」

$\hookrightarrow$ efiles:1;/opt/apps/intel18/impi18_0/modulefiles:

$\hookrightarrow \quad 1$;/opt/apps/intel18/modulefiles:1;/opt/apps/xsed

$\hookrightarrow$ e/modulefiles:1;/opt/apps/modulefiles: 1 ;/opt/mod

$\hookrightarrow$ ulefiles: 1

NO_HOSTSORT $=1$

TACC_INTEL_DIR=/opt/intel/compilers_and_libraries_20 」

$\hookrightarrow$ 18.2.199/linux

TACC_LIBFABRIC_BIN=/opt/apps/libfabric/1.7.0/bin

TACC_FAMILY_QT=qt5

FFTW_ROOT=/opt/apps/intel18/impi18_0/fftw3/3.3.8

HISTSIZE $=1000$

IDEV_SETUP_BYPASS $=1.0$

SLURM_JOB_QOS=normal

I_MPI_FABRICS=shm: of $\mathrm{i}$

TACC_IMPI_BIN=/opt/intel/compilers_and_libraries_201」

$\hookrightarrow$ 8.2.199/linux/mpi/intel64/bin 
VES3D_DIR=/home1/anonymous/USER/projects/boundary/ve 」 $\hookrightarrow$ S3d-cXX

SSH_CLIENT=206.76.192.54 4739422

LMOD_SYSTEM_DEFAULT_MODULES=TACC

TMPDIR $=/$ tmp

SLURM_TOPOLOGY_ADDR_PATTERN=node

TACC_LIBFABRIC_DIR=/opt/apps/libfabric/1.7.0

PETSC_ARCH=skylake $-i 64$

QT_QPA_PLATFORM_PLUGIN_PATH=/opt/apps/qt5/5.11.2/plu 」

$\hookrightarrow$ gins

XALT_DIR=/opt/apps/xalt/xalt/

LIBRARY_PATH=/opt/intel/compilers_and_libraries_2018」

$\hookrightarrow$.2.199/linux/daal/../tbb/lib/intel64_lin/gcc4.4: 」

$\hookrightarrow$ /opt/intel/compilers_and_libraries_2018.2.199/1i 」

$\hookrightarrow$ nux/daal/lib/intel64_lin:/opt/intel/compilers_an」

$\hookrightarrow$ d_libraries_2018.2.199/linux/tbb/lib/intel64/gcc」

$\hookrightarrow$ 4.7:/opt/intel/compilers_and_libraries_2018.2.19」

$\hookrightarrow$ 9/linux/mkl/lib/intel64_lin:/opt/intel/compilers」

$\hookrightarrow$ _and_libraries_2018.2.199/linux/compiler/lib/int 」

$\hookrightarrow$ el64_lin:/opt/intel/compilers_and_libraries_2018」

$\hookrightarrow .2 .199 /$ linux/ipp/lib/intel64

TACC_LIBFABRIC_INC=/opt/apps/libfabric/1.7.0/include

TACC_BOOST_BIN=/opt/apps/intel18/boost/1.68/bin

LMOD_PKG=/opt/apps/lmod/lmod

TACC_FAMILY_COMPILER_VERSION $=18.0 .2$

TACC_FAMILY_VTK_VERSION $=8.1 .1$

QTDIR=/opt/apps/qt5/5.11.2

TACC_P4EST_BIN=/home1/apps/intel18/impi18_0/p4est/2.」

$\hookrightarrow \quad 0 /$ bin

QTINC=/usr/lib64/qt-3.3/include

LMOD_VERSION $=7.8 .21$

SSH_TTY=/dev/pts/0

SLURM_TACC_RUNLIMIT_MINS $=30$

LC_ALL=en_US.UTF-8

__LMOD_REF_COUNT_LOADEDMODULES $=$ intel $/ 18.0 .2: 1 ; 1 \mathrm{ibfab}$ 」

$\hookrightarrow \mathrm{ric} / 1.7 .0: 1 ; \mathrm{impi} / 18.0 .2: 1 ; \mathrm{fftw} 3 / 3.3 .8: 1 ; \mathrm{petsc} / 3$.

$\hookrightarrow \quad 10-\mathrm{i} 64: 1 ;$ boost/1.68:1;qt5/5.11.2:1;vtk/8.1.1:1;

$\hookrightarrow$ 4est/2.0:1

I_MPI_JOB_FAST_STARTUP $=1$

VES3D_PLATFORM=stampede

FFTW_DIR=/opt/apps/intel18/impi18_0/fftw3/3.3.8

FACEMAP_DIR=/home1/anonymous/USER/projects/boundary/ 」

$\hookrightarrow$ mobo-temp/face_map

QT_GRAPHICSSYSTEM_CHECKED $=1$

TACC_INTEL_BIN=/opt/intel/compilers_and_libraries_20 」

$\hookrightarrow$ 18.2.199/linux/bin/intel64

TACC_IMPI_INC=/opt/intel/compilers_and_libraries_201 」 $\hookrightarrow$ 8.2.199/linux/mpi/intel64/include

TACC_FFTW3_DIR=/opt/apps/intel18/impi18_0/fftw3/3.3.8

USER=USER

SLURM_NNODES $=1$

IDEV_QDEL=scancel

__LMOD_REF_COUNT_QT_QPA_PLATFORM_PLUGIN_PATH=/opt/ap 」

$\hookrightarrow$ ps/qt5/5.11.2/plugins: 1
LS_COLORS $=\mathrm{rs}=0: \mathrm{di}=38 ; 5 ; 27: \mathrm{ln}=38 ; 5 ; 51: \mathrm{mh}=44 ; 38 ; 5 ; 15: \mathrm{p}$ ।

$\hookrightarrow \quad \mathrm{i}=40 ; 38 ; 5 ; 11: \mathrm{so}=38 ; 5 ; 13: \mathrm{do}=38 ; 5 ; 5: \mathrm{bd}=48 ; 5 ; 232 ; 38$ 」

$\hookrightarrow \quad ; 5 ; 11: \mathrm{cd}=48 ; 5 ; 232 ; 38 ; 5 ; 3:$ or $=48 ; 5 ; 232 ; 38 ; 5 ; 9: \mathrm{mi}=0$ 」

$\hookrightarrow \quad 5 ; 48 ; 5 ; 232 ; 38 ; 5 ; 15: \mathrm{su}=48 ; 5 ; 196 ; 38 ; 5 ; 15: \mathrm{sg}=48 ; 5 ; 1$ 」

$\hookrightarrow \quad 1 ; 38 ; 5 ; 16: \mathrm{ca}=48 ; 5 ; 196 ; 38 ; 5 ; 226: \mathrm{tw}=48 ; 5 ; 10 ; 38 ; 5 ; 1$ 」

$\hookrightarrow \quad 6: \mathrm{ow}=48 ; 5 ; 10 ; 38 ; 5 ; 21: \mathrm{st}=48 ; 5 ; 21 ; 38 ; 5 ; 15: \mathrm{ex}=38 ; 5 ;$ 」

$\hookrightarrow \quad 34: * . \operatorname{tar}=38 ; 5 ; 9: * . \operatorname{tgz}=38 ; 5 ; 9: * . \operatorname{arc}=38 ; 5 ; 9: * . \operatorname{arj}=$ 」

$\hookrightarrow \quad 38 ; 5 ; 9: *$. taz $=38 ; 5 ; 9: * .1$ ha $=38 ; 5 ; 9: * .1 z 4=38 ; 5 ; 9: *$.

$\hookrightarrow \quad l z h=38 ; 5 ; 9: * .1 z m a=38 ; 5 ; 9: * . t l z=38 ; 5 ; 9: * . t x z=38 ; 5$ 」

$\hookrightarrow \quad ; 9: * . t z o=38 ; 5 ; 9: * . t 7 z=38 ; 5 ; 9: * . z i p=38 ; 5 ; 9: * . z=38$ ।

$\hookrightarrow \quad ; 5 ; 9: * . Z=38 ; 5 ; 9: * . d z=38 ; 5 ; 9: * . g z=38 ; 5 ; 9: * .1 r z=38$ 」

$\hookrightarrow \quad ; 5 ; 9: * .1 z=38 ; 5 ; 9: * .1 z o=38 ; 5 ; 9: * . x z=38 ; 5 ; 9: * . b z 2=$ 」

$\hookrightarrow \quad 38 ; 5 ; 9: * . b z=38 ; 5 ; 9: * . t b z=38 ; 5 ; 9: * . t b z 2=38 ; 5 ; 9: *$.

$\hookrightarrow \quad \mathrm{tz}=38 ; 5 ; 9: * . \mathrm{deb}=38 ; 5 ; 9: * . \mathrm{rpm}=38 ; 5 ; 9: *$. jar $=38 ; 5 ; 9$ 」

$\hookrightarrow \quad: *$.war $=38 ; 5 ; 9: *$. ear $=38 ; 5 ; 9: *$. sar $=38 ; 5 ; 9: *$. rar $=38$ 」

$\hookrightarrow \quad ; 5 ; 9: *$.alz $=38 ; 5 ; 9: *$. ace $=38 ; 5 ; 9: * . z 00=38 ; 5 ; 9: * . c p$ ।

$\hookrightarrow \quad$ io $=38 ; 5 ; 9: * .7 z=38 ; 5 ; 9: * . r z=38 ; 5 ; 9: * . c a b=38 ; 5 ; 9: *$ J

$\hookrightarrow$.jpg $=38 ; 5 ; 13: *$.jpeg $=38 ; 5 ; 13: *$.gi $f=38 ; 5 ; 13: * . b m p=$ 」

$\hookrightarrow \quad 38 ; 5 ; 13: * . p b m=38 ; 5 ; 13: *$.pgm $=38 ; 5 ; 13: * . p p m=38 ; 5 ; 1$ 」

$\hookrightarrow \quad 3: *$.tga $=38 ; 5 ; 13: * . x b m=38 ; 5 ; 13: * . x p m=38 ; 5 ; 13: *$.ti 」

$\hookrightarrow \mathrm{f}=38 ; 5 ; 13: *$.tiff $=38 ; 5 ; 13: *$.png $=38 ; 5 ; 13: *$.svg=38; ।

$\hookrightarrow \quad 5 ; 13: *$. svgz $=38 ; 5 ; 13: *$.mng $=38 ; 5 ; 13: * . p c x=38 ; 5 ; 13:$ 」

$\hookrightarrow \quad$ *.mov $=38 ; 5 ; 13: * . \mathrm{mpg}=38 ; 5 ; 13: * . \mathrm{mpeg}=38 ; 5 ; 13: * . \mathrm{m} 2 \mathrm{v}$ 」

$\hookrightarrow \quad=38 ; 5 ; 13: * . m k v=38 ; 5 ; 13: *$. webm $=38 ; 5 ; 13: *$ ogm $=38 ; 5$ 」

$\hookrightarrow \quad ; 13: * . \mathrm{mp} 4=38 ; 5 ; 13: * . \mathrm{m} 4 \mathrm{v}=38 ; 5 ; 13: * . \mathrm{mp} 4 \mathrm{v}=38 ; 5 ; 13: *$ 」

$\hookrightarrow \quad$ vob $=38 ; 5 ; 13: *$.qt $=38 ; 5 ; 13: *$.nuv $=38 ; 5 ; 13: *$. wmv $=38$ 」

$\hookrightarrow \quad ; 5 ; 13: *$. asf $=38 ; 5 ; 13: * . r m=38 ; 5 ; 13: * . r m v b=38 ; 5 ; 13:$,

$\hookrightarrow \quad * . \mathrm{flc}=38 ; 5 ; 13: *$.avi $=38 ; 5 ; 13: * . \mathrm{fli}=38 ; 5 ; 13: * . \mathrm{flv}=$ 」

$\hookrightarrow \quad 38 ; 5 ; 13: * . g l=38 ; 5 ; 13: * . d l=38 ; 5 ; 13: * . x c f=38 ; 5 ; 13:$,

$\hookrightarrow \quad * . x w d=38 ; 5 ; 13: *$. yuv $=38 ; 5 ; 13: *$. cgm $=38 ; 5 ; 13: *$. emf $=$ 」

$\hookrightarrow \quad 38 ; 5 ; 13: *$. axv $=38 ; 5 ; 13: *$. an $x=38 ; 5 ; 13: *$. ogv $=38 ; 5 ; 1$ 」

$\hookrightarrow \quad 3: *$. ogx $=38 ; 5 ; 13: *$. aac $=38 ; 5 ; 45: *$. au $=38 ; 5 ; 45: * . \mathrm{fla}$ 」

$\hookrightarrow \mathrm{c}=38 ; 5 ; 45: *$.mid $=38 ; 5 ; 45: *$.midi $=38 ; 5 ; 45: *$.mka $=38$; 」

$\hookrightarrow 5 ; 45: * . \mathrm{mp} 3=38 ; 5 ; 45: * . \mathrm{mpc}=38 ; 5 ; 45: *$. ogg $=38 ; 5 ; 45: *$ 」

$\hookrightarrow \quad . r a=38 ; 5 ; 45: *$. wav $=38 ; 5 ; 45: * . a x a=38 ; 5 ; 45: *$. oga $=38$ 」

$\hookrightarrow \quad ; 5 ; 45: *$. spx $=38 ; 5 ; 45: * . x s p f=38 ; 5 ; 45:$ 
LD_LIBRARY_PATH=/home1/apps/intel18/impi18_0/p4est/2」 $\hookrightarrow .0 / \mathrm{ib}: /$ home1/apps/intel18/impi18_0/vtk/8.1.1/li 」

$\hookrightarrow$ b:/opt/apps/gcc/6.3.0/lib64:/opt/apps/gcc/6.3.0/」

$\hookrightarrow \quad \mathrm{ib}: / o p t / a p p s / q t 5 / 5.11 .2 / \mathrm{ib}: / o p t / a p p s / i n t e 118 / \mathrm{b}$ 」

$\hookrightarrow$ oost/1.68/lib:/home1/apps/intel18/impi18_0/petsc」

$\hookrightarrow$ /3.10/skylake-i64/lib:/opt/apps/libfabric/1.7.0/」

$\hookrightarrow \quad$ lib:/opt/intel/compilers_and_libraries_2018.2.19」

$\hookrightarrow$ 9/linux/mpi/intel64/lib:/opt/intel/debugger_2018」

$\hookrightarrow$ /libipt/intel64/lib:/opt/intel/debugger_2018/iga 」

$\hookrightarrow /$ ib:/opt/intel/compilers_and_libraries_2018.2.1」

$\hookrightarrow$ 99/linux/daal/../tbb/lib/intel64_lin/gcc4.4:/opt」

$\hookrightarrow$ /intel/compilers_and_libraries_2018.2.199/linux/」

$\hookrightarrow$ daal/lib/intel64_lin:/opt/intel/compilers_and_li」

$\hookrightarrow$ braries_2018.2.199/linux/tbb/lib/intel64/gcc4.7: 」

$\hookrightarrow$ /opt/intel/compilers_and_libraries_2018.2.199/li 」

$\hookrightarrow$ nux/mkl/lib/intel64_lin:/opt/intel/compilers_and 」

$\hookrightarrow$ _libraries_2018.2.199/linux/compiler/lib/intel64」

$\hookrightarrow$ _lin:/opt/intel/compilers_and_libraries_2018.2.1」

$\hookrightarrow$ 99/linux/ipp/lib/intel64:/opt/intel/compilers_an」

$\hookrightarrow$ d_libraries_2018.2.199/linux/compiler/lib/intel6」

$\hookrightarrow$ 4:/opt/apps/intel18/impi18_0/fftw3/3.3.8/lib:/ho」

$\hookrightarrow$ me1/anonymous/USER/installs/cgal/lib64:/home1/an 」

$\hookrightarrow$ onymous/USER/installs/gmp/lib:/home1/anonymous/U 」

$\hookrightarrow$ SER/installs/mpfr/lib:/home1/anonymous/USER/proj 」

$\hookrightarrow$ ects/boundary/contact3d/lib

TACC_QT5_DIR=/opt/apps/qt5/5.11.2

PSTLROOT=/opt/intel/compilers_and_libraries_2018.2.1 ।

$\hookrightarrow$ 99/linux/pstl

TACC_PETSC_DIR=/home1/apps/intel18/impi18_0/petsc/3. 」 $\hookrightarrow \quad 10 /$

-_TRACKER_-_ 1

XALT_DATE_TIME=2019_05_20_14_47_32_9791

TACC_NODE_TYPE $=\mathrm{knl}$

SLURM_TACC_NODES $=1$

SLURM_JOBID $=3614206$

_PERSONAL_PATH__ $=1$

CPATH=/opt/intel/compilers_and_libraries_2018.2.199/ 」

$\hookrightarrow \quad$ linux/pstl/include:/opt/intel/compilers_and_libr」

$\hookrightarrow$ aries_2018.2.199/linux/daal/include:/opt/intel/c 」

$\hookrightarrow$ ompilers_and_libraries_2018.2.199/linux/tbb/incl」

$\hookrightarrow$ ude:/opt/intel/compilers_and_libraries_2018.2.19」

$\hookrightarrow$ 9/linux/mkl/include:/opt/intel/compilers_and_lib」

$\hookrightarrow$ raries_2018.2.199/linux/ipp/include

IFC_BIN=/opt/intel/compilers_and_libraries_2018.2.19」

$\hookrightarrow \quad$ 9/linux/bin/intel64

TACC_MKL_LIB=/opt/intel/compilers_and_libraries_2018」

$\hookrightarrow \quad .2 .199 / 1$ inux/mkl/lib/intel64
_ModuleTable004_=ImxvYWRPcmRlci JdPTkscHJvcFQ9e30sWyJ

$\hookrightarrow \quad$ zdGFja0RlcHRoI109MCXbInN0YXR1cyJdPSJhY3RpdmUiLFs 」

$\hookrightarrow \quad$ idXNlck5hbWUiXT0icDRlc3QiLH0scGV0c2M9e1siZm4iXT0

$\hookrightarrow \quad$ iL29wdC9hcHBzL2ludGVsMTgvaW1waTE4XzAvbW9kdWxlZml」

$\hookrightarrow$ sZXMvcGV0c2MvMy4xMC1pNjQubHVhIixbImZ1bGxOYW11Ilo」

$\hookrightarrow$ 9InBldHNjLzMuMTAtaTYOIixbImxvYWRPcmRlciJdPTUscHJ 」

$\hookrightarrow \quad$ vcFQ9e30sWyJzdGFja0RlcHRoIl09MCXbInNOYXR1cyJdPSJ 」

$\hookrightarrow$ hY3RpdmUiLFsidXNlck5hbWUiXT0icGV0c2MvMy4XMC1pNjQ」

$\hookrightarrow \quad$ iLH0scXQ1PXtbImZuI109Ii 9vcHQvYXBwcy9tb2R1bGVmaWx 」

$\hookrightarrow \quad$ lcy9xdDUvNS4xMS4yLmx1YSIsWyJmdWxsTmFtZSJdPSJxdDU 」

$\hookrightarrow \quad$ VNS4xMS4yI i xbImxvYWRPcmRlciJdPTcscHJvcFQ9e30s

XALT_RUN_UUID=f56fe2e9-d1e7-498b-9289-9ce6973d60f2

OLDSCRATCH=/oldscratch/anonymous/USER

SLURM_COMMAND=sbatch

__LMOD_REF_COUNT__LMFILES_=/opt/apps/modulefiles/int 」

$\hookrightarrow$ el/18.0.2.lua:1;/opt/apps/modulefiles/libfabric/

$\hookrightarrow$ 1.7.0.1ua:1;/opt/apps/intel18/modulefiles/impi/1」

$\hookrightarrow$ 8.0.2.lua:1;/opt/apps/intel18/impi18_0/modulefil

$\hookrightarrow$ es/fftw3/3.3.8.lua:1;/opt/apps/intel18/impi18_0/

$\hookrightarrow$ modulefiles/petsc/3.10-i64.lua:1;/opt/apps/intel」

$\hookrightarrow$ 18/modulefiles/boost/1.68.lua:1;/opt/apps/module 」

$\hookrightarrow$ files/qt5/5.11.2.lua:1;/opt/apps/intel18/impi18_」

$\hookrightarrow 0 /$ modulefiles/vtk/8.1.1.lua:1;/opt/apps/intel18/」

$\hookrightarrow \quad$ impi18_0/modulefiles/p4est/2.0.lua: 1

TACC_BOOST_DIR=/opt/apps/intel18/boost/1.68

PVFMM_INC=/home1/anonymous/USER/installs/pvfmm/inclu 」

$\hookrightarrow$ de/pvfmm

SLURM_NTASKS=1

PETSC_ROOT=/home1/apps/intel18/impi18_0/petsc/3.10/

SLURM_TACC_JOBNAME $=i d v 63880$

LMOD_FAMILY_MPI_VERSION $=18.0 .2$

TACC_PETSC_LIB=/home1/apps/intel18/impi18_0/petsc/3. 」

$\hookrightarrow$ 10/skylake-i64/lib

ARCHIVER=ranch. tacc . utexas. edu

NLSPATH=/opt/intel/debugger_2018/gdb/intel64/share/1」

$\hookrightarrow \quad$ ocale/\%1_\%t/\%N:/opt/intel/compilers_and_librarie 」

$\hookrightarrow$ s_2018.2.199/linux/mkl/lib/intel64/locale/\%1_\%t/」

$\hookrightarrow \% \mathrm{~N}$ : /opt/intel/compilers_and_libraries_2018.2.199

$\hookrightarrow /$ linux/compiler/lib/intel64/locale/\%1_\%t/\%N

__LMOD_REF_COUNT_I_MPI_ROOT=/opt/intel/compilers_and 」

$\hookrightarrow$ _libraries_2018.2.199/1inux/mpi:1

LMOD_FAMILY_QT_VERSION $=5.11 .2$

TACC_QT5_BIN=/opt/apps/qt5/5.11.2/bin

$\mathrm{PATH}=/$ home1/apps/intel18/impi18_0/p4est/2.0/bin:/hom 」

$\hookrightarrow$ e1/apps/intel18/impi18_0/vtk/8.1.1/bin:/opt/apps

$\hookrightarrow$ /gcc/6.3.0/bin:/opt/apps/qt5/5.11.2/bin:/opt/app 」

$\hookrightarrow$ s/intel18/boost/1.68/bin:/home1/apps/intel18/imp」

$\hookrightarrow \quad$ i18_0/petsc/3.10/skylake-i64/bin:/opt/apps/libfa 」

$\hookrightarrow$ bric/1.7.0/bin:/opt/apps/intel18/impi/18.0.2/bin」

$\hookrightarrow \quad:$ /opt/intel/compilers_and_libraries_2018.2.199/1」

$\hookrightarrow$ inux/mpi/intel64/bin:/opt/intel/compilers_and_li」

$\hookrightarrow$ braries_2018.2.199/linux/bin/intel64:/usr/lib64/

$\hookrightarrow$ qt-3.3/bin:/usr/local/bin:/bin:/usr/bin:/opt/del

$\hookrightarrow \quad$ 1/srvadmin/bin:/opt/apps/intel18/impi18_0/fftw3/」

$\hookrightarrow \quad 3.3 .8 / \mathrm{bin}$

MAIL=/var/spool/mail/USER 
TACC_SYSTEM=stampede2

_ModuleTable001_=X01vZHVsZVRhYmxIXz17WyJNVHZlcnNpb24」

$\hookrightarrow \quad$ iXTOzLFsiY19yZWJ1aWxkVGltZSJdPWZhbHN1LFsiY19zaG9 」

$\hookrightarrow \quad$ ydFRpbWUiXT1mYWxzZSxkZXB0aFQ9e30sZmFtaWx5PXtbIk1」

$\hookrightarrow$ QSSJdPSJpbXBpI ixbImNvbXBpbGVyIl09ImludGVsIixbInF 」

$\hookrightarrow$ 0I109InF0NSIsWyJ2dGsiXT0idnRrIix9LG1UPXtib29zdD1」

$\hookrightarrow \quad$ 7WyJmbi JdPSIvb3B0L2FwcHMvaW50ZWwxOC9tb2R1bGVmaWx

$\hookrightarrow \quad$ lcy9ib29zdC8xLjY4Lmx1YSIsWyJmdWxsTmFtZSJdPSJib29」

$\hookrightarrow \quad z d C 8 x L j Y 4 I i x b I m x v Y W R P c m R l c i J d P T Y s c H J v c F Q 9 e 30 s W y J$

$\hookrightarrow \quad$ zdGFja0RlcHRoIl09MCXbInN0YXR1cyJdPSJhY3RpdmUiLFs 」

$\hookrightarrow \quad$ idXNlck5hbWUiXT0iYm9vc3QiLH0sZmZ0dzM9e1siZm4iXT0 」

$\hookrightarrow \quad$ iL29wdC9hcHBzL2ludGVsMTgvaW1waTE4XzAvbW9kdWxl

SLURM_TASKS_PER_NODE $=1$

ICC_BIN=/opt/intel/compilers_and_libraries_2018.2.19 」

$\hookrightarrow \quad 9 /$ linux/bin/intel64

__LMOD_REF_COUNT_NLSPATH=/opt/intel/debugger_2018/gd 」

$\hookrightarrow$ b/intel64/share/locale/\%1_\%t/\%N:1;/opt/intel/com」

$\hookrightarrow$ pilers_and_libraries_2018.2.199/linux/mkl/lib/in」

$\hookrightarrow$ tel64/locale/\%1_\%t/\%N:1;/opt/intel/compilers_and

$\hookrightarrow$ _libraries_2018.2.199/linux/compiler/lib/intel64」

$\hookrightarrow /$ locale/\%1_\%t/\%N: 1

I_MPI_OFI_LIBRARY=/opt/apps/libfabric/1.7.0/lib/libf 」

$\hookrightarrow$ abric.so

STOCKYARD=/work/anonymous/USER

RUNNING_IDEV $=1$

SLURM_WORKING_CLUSTER=stampede2:206.76.192.2:6820:84 」

$\hookrightarrow \quad 48$

GEOGRAM_DIR=/home1/anonymous/USER/projects/boundary/ 」

$\hookrightarrow$ mobo-temp/geogram_1.6.3

$=/ \mathrm{bin} / \mathrm{env}$

WORK=/work/anonymous/USER/stampede 2

TACCINFO=/usr $/$ local/etc/taccinfo

SLURM_JOB_ID $=3614206$

TACC_VTK_LIB=/home1/apps/intel18/impi18_0/vtk/8.1.1/ 」 $\hookrightarrow \quad l i b$

OLDWORK=/work/anonymous/USER

TBBROOT=/opt/intel/compilers_and_libraries_2018.2.19」

$\hookrightarrow \quad 9 /$ linux/tbb

TACC_LIBFABRIC_LIB=/opt/apps/libfabric/1.7.0/lib

$\mathrm{PWD}=/$ home $1 /$ anonymous /USER

INPUTRC=/etc/inputrc

SLURM_JOB_USER=USER

SLURM_QUEUE=development

SLURM_TACC_NCORES_SET $=1$

_LMFILES_=/opt/apps/modulefiles/intel/18.0.2.lua:/op 」

$\hookrightarrow$ t/apps/modulefiles/libfabric/1.7.0.lua:/opt/apps」

$\hookrightarrow /$ intel18/modulefiles/impi/18.0.2.lua:/opt/apps/i 」

$\hookrightarrow$ ntel18/impi18_0/modulefiles/fftw3/3.3.8.lua:/opt 」

$\hookrightarrow$ /apps/intel18/impi18_0/modulefiles/petsc/3.10-i6」

$\hookrightarrow$ 4.lua:/opt/apps/intel18/modulefiles/boost/1.68.1」

$\hookrightarrow$ ua:/opt/apps/modulefiles/qt5/5.11.2.lua:/opt/app」

$\hookrightarrow$ s/intel18/impi18_0/modulefiles/vtk/8.1.1.1ua:/op

$\hookrightarrow$ t/apps/intel18/impi18_0/modulefiles/p4est/2.0.lua

TACC_P4EST_DIR=/home1/apps/intel18/impi 18_0/p4est/2.0

LANG=en_US.UTF-8
__LMOD_REF_COUNT_PYTHONPATH=/home1/apps/intel18/impi 」 $\hookrightarrow$ 18_0/vtk/8.1.1/lib/site-packages/mpi4py: 1 ;/home 1 」

$\hookrightarrow$ /apps/intel18/impi18_0/vtk/8.1.1/lib/python2.7/s 」

$\hookrightarrow$ ite-packages/vtk: 1

HEDGEHOG_CC=mpicc

VTK_INC_DIR=/include/vtk-8.1/

MODULEPATH=/opt/apps/qt5.11.2/modulefiles:/opt/apps/」

$\hookrightarrow$ intel18/impi18_0/modulefiles:/opt/apps/intel18/m」

$\hookrightarrow$ odulefiles:/opt/apps/xsede/modulefiles:/opt/apps」

$\hookrightarrow$ /modulefiles:/opt/modulefiles

_ModuleTable_Sz_=6

SLURM_JOB_UID $=846024$

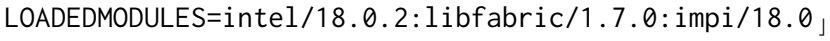

$\hookrightarrow \quad .2: \mathrm{fftw} 3 / 3.3 .8:$ petsc/3.10-i64:boost/1.68: qt5/5.1 」

$\hookrightarrow \quad 1.2: \mathrm{vtk} / 8.1 .1: \mathrm{p} 4 \mathrm{est} / 2.0$

SLURM_NODEID $=0$

idev_has_user_PERL5LIB=no

_-BASHRC_SOURCED__ $=1$

TACC_VTK_DIR=/home1/apps/intel18/impi18_o/vtk/8.1.1

SLURM_SUBMIT_DIR=/home $1 /$ anonymous/USER

TACC_VEC_FLAGS $=-x$ CORE-AVX2 -axCORE-AVX512, MIC-AVX512

I_MPI_F90 $=$ ifort

LMOD_CMD=/opt/apps/lmod/lmod/libexec/lmod

SLURM_TASK_PID $=239883$

SLURM_NPROCS $=1$

I_MPI_CC $=$ icC

_ModuleTable005_=WyJzdGFja0RlcHRoII09MCXbInN0YXR1cyJ 」

$\hookrightarrow$ dPSJhY3RpdmUiLFsidXNlck5hbWUiXT0icXQ1 Iix9LHZOaz1 」

$\hookrightarrow$ 7WyJmbi JdPSIvb3B0L2FwcHMvaW50ZWwXOC9pbXBpMThfMC9 」

$\hookrightarrow$ tb2R1bGVmaWxlcy92dGsvOC4xLjEubHVhIixbImZ1bGxOYW1」

$\hookrightarrow \quad$ 1Il09InZ0ay84LjEuMSIsWyJsb2FkT3JkZXIiXT04LHByb3B 」

$\hookrightarrow$ UPXt9LFsic3RhY2tEZXBOaCJdPTAsWyJzdGFOdXMiXTOiYWN

$\hookrightarrow$ 0aXZlIixbInVzZXJOYW11Il09InZ0ayIsfSX9LG1wYXRoQT1」

$\hookrightarrow$ 7Ii9vcHQvYXBwcy9xdDUuMTEuMi9tb2R1bGVmaWxlcyIsIi 9 」

$\hookrightarrow \quad$ vcHQvYXBwcy9pbnRIbDE4L21tcGkxOF 8wL21vZHVsZWZpbGV」

$\hookrightarrow \quad$ zIiwiL29wdC9hcHBzL2ludGVsMTgvbW9kdWxlZmlsZXMiLCI 」

$\hookrightarrow \quad$ vb3B0L2FwcHMveHN1ZGUvbW9kdWxlZmlsZXMiLCIvb3B0

MOBO_DIR=/home1/anonymous/USER/projects/boundary/mob」

$\hookrightarrow \quad$ o-temp

SLURM_CPUS_ON_NODE $=272$

DAALROOT=/opt/intel/compilers_and_libraries_2018.2.1」 $\hookrightarrow$ 99/linux/daal

TACC_P4EST_LIB=/home1/apps/intel18/impi18_0/p4est/2. 」

$\hookrightarrow \quad 0 / 1 \mathrm{ib}$

SSH_ASKPASS=/usr/libexec/openssh/gnome-ssh-askpass

SLURM_PROCID $=0$

ENVIRONMENT $=\mathrm{BATCH}$

TACC_MKL_INC=/opt/intel/compilers_and_libraries_2018」

$\hookrightarrow \quad$.2.199/linux/mkl/include

SLURM_JOB_NODELIST $=c 455-061$

HOME $=/$ home 1 /anonymous/USER

SHLVL=4

FI_PROVIDER=psm2

TACC_DOMAIN=stampede 2

SLURM_LOCALID $=0$

__XALT_INITIAL_STATE_-_LD_PRELOAD 
LANGUAGE=en_US. UTF-8

__LMOD_REF_COUNT_PATH=/home1/apps/intel18/impi18_0/p 」

$\hookrightarrow$ 4est/2.0/bin:1;/home1/apps/intel18/impi18_0/vtk/」

$\hookrightarrow \quad 8.1 .1 / \mathrm{bin}: 1 ; /$ opt/apps/gcc/6.3.0/bin:2;/opt/apps/」

$\hookrightarrow$ qt5/5.11.2/bin:1;/opt/apps/intel18/boost/1.68/bi」

$\hookrightarrow \mathrm{n}: 1 ;$ /home1/apps/intel18/impi18_0/petsc/3.10/skyl」

$\hookrightarrow$ ake-i64/bin:1;/opt/apps/libfabric/1.7.0/bin:1;/o」

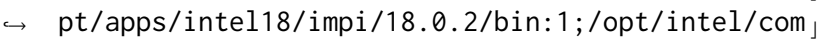

$\hookrightarrow$ pilers_and_libraries_2018.2.199/linux/mpi/intel6」

$\hookrightarrow$ 4/bin:1;/opt/intel/compilers_and_libraries_2018.」

$\hookrightarrow$ 2.199/linux/bin/intel64:1;/usr/lib64/qt-3.3/bin:

$\hookrightarrow \quad 1$;/usr/local/bin: 1 ;/bin: 1 ;/usr/bin: 1 ;/opt/dell/s

$\hookrightarrow$ rvadmin/bin:1;/opt/apps/intel18/impi18_0/fftw3/3」

$\hookrightarrow \quad .3 .8 / \mathrm{bin}: 1$

TACC_FAMILY_COMPILER=intel

TACC_INTEL_LIB=/opt/intel/compilers_and_libraries_20,

$\hookrightarrow$ 18.2.199/linux/compiler/lib/intel64

I_MPI_CXX=icpc

TACC_PETSC_INC=/home1/apps/intel18/impi18_0/petsc/3.」 $\hookrightarrow$ 10/skylake-i64/include

TACC_FAMILY_VTK=vtk

__LMOD_REF_COUNT_CPATH=/opt/intel/compilers_and_libr 」

$\hookrightarrow$ aries_2018.2.199/linux/pstl/include:1;/opt/intel」

$\hookrightarrow$ /compilers_and_libraries_2018.2.199/linux/daal/i 」

$\hookrightarrow$ nclude:1;/opt/intel/compilers_and_libraries_2018」

$\hookrightarrow \quad$.2.199/linux/tbb/include:1;/opt/intel/compilers_」

$\hookrightarrow$ and_libraries_2018.2.199/linux/mkl/include:1;/op」

$\hookrightarrow \quad$ t/intel/compilers_and_libraries_2018.2.199/linux 」

$\hookrightarrow$ /ipp/include: 1

TACC_BOOST_LIB=/opt/apps/intel18/boost/1.68/lib

TACC_P4EST_INC=/home1/apps/intel18/impi18_0/p4est/2. 」 $\hookrightarrow \quad 0 /$ include

_ModuleTable002_=ZmlsZXMvZmZ0dzMvMy4zLjgubHVhIixbImZ」

$\hookrightarrow$ 1bGx0YW11Il09ImZmdHczLzMuMy44IixbImxvYWRPcmRIci J

$\hookrightarrow$ dPTQscHJvcFQ9e30sWyJzdGFja0RIcHRoI109MCxbInNOYXR」

$\hookrightarrow$ 1cyJdPSJhY3RpdmUiLFsidXNlck5hbWUiXTOiZmZOdzMiLHO」

$\hookrightarrow$ saW1waT17Wy Jmbi JdPSIvb3B0L2FwcHMvaW50ZWwxOC9tb2R 」

$\hookrightarrow$ 1bGVmaWxlcy9pbXBpLzE4LjAuMi5sdWEiLFsiZnVsbE5hbWU 」

$\hookrightarrow \quad$ iXT0iaW1waS8xOC4wLjIiLFsibG9hZE9yZGVyIl09Myxwcm9 」

$\hookrightarrow \quad$ WVD17fSxbInNOYWNrRGVwdGgiXTOWLFsic3RhdHVzII09ImF 」

$\hookrightarrow$ jdG12ZSIsWyJ1c2VyTmFtZSJdPSJpbXBpI ix9LGludGVsPXt

$\hookrightarrow$ bImZuIl09Ii9vcHQvYXBwcy9tb2R1bGVmaWxlcy9pbnRlbc8

$\hookrightarrow \quad$ xOC4wLjIubHVhIixbImZ1bGxOYW11I109ImludGVsLzE4

SLURM_CLUSTER_NAME=stampede 2

SLURM_JOB_CPUS_PER_NODE $=272$

SLURM_JOB_GID $=814474$

IFC_LIB=/opt/intel/compilers_and_libraries_2018.2.19」

$\hookrightarrow$ 9/linux/compiler/lib/intel64

SLURM_GTIDS $=0$

SLURM_SUBMIT_HOST=login4.stampede2. tacc.utexas.edu

__LMOD_REF_COUNT_INCLUDE=/home1/apps/intel18/impi 18_ 」

$\hookrightarrow \quad 0 / v t k / 8.1 .1 /$ include: 1 ;/opt/apps/gcc/6.3.0/includ 」

$\hookrightarrow \mathrm{e}: 1$

BLENDSURF_DIR=/home1/anonymous/USER/projects/boundar 」

$\hookrightarrow y / m o b o-t e m p / b l e n d s u r f 3$

BASH_ENV=/etc/tacc/tacc_functions idev_ip $=\mathrm{c} 455-061$

SLURM_JOB_PARTITION=development

I_MPI_FC=ifort

TACC_VTK_INC=/home1/apps/intel18/impi18_0/vtk/8.1.1/ 」 $\hookrightarrow$ include

PVFMM_LIB=/home1/anonymous/USER/installs/pvfmm/lib/p 」

$\hookrightarrow \quad \mathrm{Vfmm}$

LOGNAME $=$ USER

ICC_LIB=/opt/intel/compilers_and_libraries_2018.2.19」

$\hookrightarrow$ 9/linux/compiler/lib/intel64

TACC_FAMILY_MPI =impi

LMOD_FAMILY_VTK=vtk

PYTHONPATH=/home1/apps/intel18/impi18_0/vtk/8.1.1/li 」

$\hookrightarrow$ b/site-packages/mpi4py:/home1/apps/intel18/impi1

$\hookrightarrow$ 8_0/vtk/8.1.1/1ib/python2.7/site-packages/vtk

CVS_RSH=ssh

QTLIB=/usr/lib64/qt-3.3/lib

LMOD_SETTARG_TITLE_BAR=yes

BOOST_ROOT=/opt/apps/intel18/boost/1.68

SSH_CONNECTION=206.76.192.54 47394 206.76.206.122

XDG_DATA_DIRS=/home1/anonymous/USER/. local/share/fla 」

$\hookrightarrow$ tpak/exports/share:/var/lib/flatpak/exports/shar

$\hookrightarrow$ e:/usr/local/share:/usr/share

LC_CTYPE $=U T F-8$

SLURM_JOB_ACCOUNT=TG-DPP130002

HEDGEHOG_CXX=mpicXX

MODULESHOME $=/$ opt $/$ apps $/ 1 \mathrm{mod} / 1 \mathrm{mod}$

SLURM_JOB_NUM_NODES $=1$

__LMOD_REF_COUNT_LIBRARY_PATH=/opt/intel/compilers_a

$\hookrightarrow$ nd_libraries_2018.2.199/linux/daal/../tbb/lib/in

$\hookrightarrow$ tel64_lin/gcc4.4:1;/opt/intel/compilers_and_libr」

$\hookrightarrow$ aries_2018.2.199/linux/daal/lib/intel64_lin:1;/o」

$\hookrightarrow$ pt/intel/compilers_and_libraries_2018.2.199/linu」

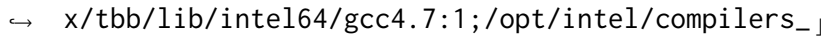

$\hookrightarrow$ and_libraries_2018.2.199/linux/mkl/lib/intel64_l」

$\hookrightarrow$ in:1;/opt/intel/compilers_and_libraries_2018.2.1」

$\hookrightarrow$ 99/linux/compiler/lib/intel64_lin:1;/opt/intel/c 」

$\hookrightarrow$ ompilers_and_libraries_2018.2.199/linux/ipp/lib/

$\hookrightarrow$ intel64:1

MPI_HOME=/opt/intel/compilers_and_libraries_2018.2.1 」

$\hookrightarrow 99 /$ linux/mpi

LESSOPEN=| $/$ usr $/$ bin/lesspipe. sh \%s

LMOD_SETTARG_FULL_SUPPORT $=$ full

OMP_NUM_THREADS $=48$ 
__LMOD_REF_COUNT_LD_LIBRARY_PATH=/home1/apps/intel18 」 $\hookrightarrow$ /impi18_0/p4est/2.0/lib:1;/home1/apps/intel18/im」 $\hookrightarrow$ pi18_0/vtk/8.1.1/lib:1;/opt/apps/gcc/6.3.0/lib64」 $\hookrightarrow \quad: 2 ; /$ opt/apps/gcc/6.3.0/lib:2;/opt/apps/qt5/5.11.」 $\hookrightarrow$ 2/lib:1;/opt/apps/intel18/boost/1.68/lib:1;/home 」 $\hookrightarrow$ 1/apps/intel18/impi18_0/petsc/3.10/skylake-i64/1」 $\hookrightarrow \quad \mathrm{ib}: 1$;/opt/apps/libfabric/1.7.0/lib:1;/opt/intel/」 $\hookrightarrow$ compilers_and_libraries_2018.2.199/linux/mpi/int 」 $\hookrightarrow$ el64/lib:1;/opt/intel/debugger_2018/libipt/intel」 $\hookrightarrow$ 64/lib:1;/opt/intel/debugger_2018/iga/lib:1;/opt 」 $\hookrightarrow$ /intel/compilers_and_libraries_2018.2.199/linux/ 」 $\hookrightarrow$ daal/../tbb/lib/intel64_lin/gcc4.4:1;/opt/intel/」 $\hookrightarrow$ compilers_and_libraries_2018.2.199/linux/daal/li J $\hookrightarrow$ b/intel64_lin:1;/opt/intel/compilers_and_librari 」 $\hookrightarrow$ es_2018.2.199/linux/tbb/lib/intel64/gcc4.7:1;/op

$\hookrightarrow$ t/intel/compilers_and_libraries_2018.2.199/linux 」

$\hookrightarrow$ /mkl/lib/intel64_lin:1;/opt/intel/compilers_and_」

$\hookrightarrow$ libraries_2018.2.199/linux/compiler/lib/intel64_」

$\hookrightarrow \quad$ lin:2;/opt/intel/compilers_and_libraries_2018.2.」

$\hookrightarrow$ 199/linux/ipp/lib/intel64:1;/opt/intel/compilers」

$\hookrightarrow$ _and_libraries_2018.2.199/linux/compiler/lib/int」

$\hookrightarrow$ el64:1;/opt/apps/intel18/impi18_0/fftw3/3.3.8/li 」

$\hookrightarrow \mathrm{b}: 1$

PKG_CONFIG_PATH=/opt/intel/compilers_and_libraries_2

$\hookrightarrow$ 018.2.199/linux/mkl/bin/pkgconfig:/opt/apps/inte 」

$\hookrightarrow$ 118/impi18_0/fftw3/3.3.8/lib/pkgconfig

HEDGEHOG_DIR=/home1/anonymous/USER/projects/boundary 」

$\hookrightarrow \quad /$ mobo-temp

TACC_OVERRIDE_PROJECT $=$ TG-DPP130002

PROMPT_COMMAND $=\$\{$ X_SET_TITLE_BAR: $::\}$

$\hookrightarrow \quad$ "\$USER@\$ SHOST $\}: \$\{\mathrm{PWD} / \#$ \$HOME $/ \sim$ \}"

__Init_Default_Modules=1

TACC_FFTW3_LIB=/opt/apps/intel18/impi18_0/fftw3/3.3.」 $\hookrightarrow \quad 8 / 1 \mathrm{ib}$

LMOD_FAMILY_COMPILER=intel

TACC_IMPI_LIB=/opt/intel/compilers_and_libraries_201 」

$\hookrightarrow$ 8.2.199/linux/mpi/intel64/lib

TACC_VTK_BIN=/home1/apps/intel18/impi18_0/vtk/8.1.1/ 」 $\hookrightarrow$ bin

VTK_LOCATION=/home1/apps/intel18/impi18_0/vtk/8.1.1

XDG_RUNTIME_DIR=/run/user $/ 846024$

ARCHIVE=/home/anonymous/USER

OLDHOME=/oldhome1/anonymous/USER

IDEV_PWD=/home1/anonymous/USER

__LMOD_REF_COUNT_INTEL_LICENSE_FILE=/home 1 /anonymous 」

$\hookrightarrow$ /USER/intel/licenses: 1 ;/opt/intel/licenses: 1 ;/op ]

$\hookrightarrow \quad t /$ intel/compilers_and_libraries_2018.2.199/linux 」

$\hookrightarrow /$ licenses: 1

TACC_FAMILY_MPI_VERSION=18.0.2

__LMOD_REF_COUNT_PKG_CONFIG_PATH=/opt/intel/compiler 」

$\hookrightarrow$ S_and_libraries_2018.2.199/linux/mkl/bin/pkgconf 」

$\hookrightarrow \quad$ ig: 1 ;/opt/apps/intel18/impi18_0/fftw3/3.3.8/1ib/」

$\hookrightarrow$ pkgconfig: 1

TACC_QT5_LIB=/opt/apps/qt5/5.11.2/1ib

LMOD_FAMILY_VTK_VERSION=8.1.1
PVFMM_DIR=/home1/anonymous/USER/installs/pvfmm/share 」 $\hookrightarrow \quad / p v f m m$

LMOD_DIR=/opt $/$ apps $/ 1 \mathrm{mod} / 1 \mathrm{mod} / 1 \mathrm{ibexec}$

__LMOD_REF_COUNT_MANPATH=/opt/apps/libfabric/1.7.0/s 」

$\hookrightarrow$ hare/man:1;/opt/intel/compilers_and_libraries_20」

$\hookrightarrow$ 18.2.199/linux/mpi/man:1;/opt/intel/documentatio」

$\hookrightarrow$ n_2018/en/man/common:1;/opt/intel/documentation_」

$\hookrightarrow$ 2018/en/debugger/gdb-igfx/man: 1 ;/opt/intel/docum 」

$\hookrightarrow$ entation_2018/en/debugger/gdb-ia/man:1;/opt/apps 」

$\hookrightarrow$ /intel18/impi18_0/fftw3/3.3.8/man:1

INCLUDE=/home $1 /$ apps/intel18/impi18_0/vtk/8.1.1/inclu 」 $\hookrightarrow$ de:/opt/apps/gcc/6.3.0/include

_ModuleTable006_=L2FwcHMvbW9kdWx1ZmlsZXMiLCIvb3B0L21」

$\hookrightarrow \quad$ VZHVsZWZpbGVZIix9LFsic3lzdGVtQmFzZU1QQVRII109Ii9

$\hookrightarrow \quad$ vcHQvYXBwcy94c2VkZS9tb2R1bGVmaWxlczovb3B0L2FwcHM 」

$\hookrightarrow$ vbW9kdWx1ZmlsZXM6L29wdC9tb2R1bGVmaWxlcyIsfQ==

PETSC_DIR=/home1/apps/intel18/impi18_0/petsc/3.10/

LMOD_FAMILY_QT =qt5

SCRATCH=/scratch/anonymous/USER

SLURM_TACC_NNODES_SET $=1$

SLURM_TACC_CORES $=1$

TACC_MKL_DIR=/opt/intel/compilers_and_libraries_2018 」

$\hookrightarrow \quad .2 .199 / \mathrm{linux} / \mathrm{mkl}$

FI_PSM2_LAZY_CONN=1

LMOD_FAMILY_MPI $=$ impi

TACC_MPI_GETMODE=impi_hydra

I_MPI_ROOT=/opt/intel/compilers_and_libraries_2018.2 」

$\hookrightarrow .199 /$ linux/mpi

TACC_QT5_INC=/opt/apps/qt5/5.11.2/include

MACHINE_NAME=stampede

BASH_FUNC_sbatch ()=() $\{$ echo -e "InNOTIFICATION:

$\hookrightarrow$ sbatch not available on compute nodes. Use a login

$\hookrightarrow$ node. $\backslash$ n"

\}

BASH_FUNC_module ()$=()\{$ if $[-z$

$\hookrightarrow \quad$ "\$\{LMOD_SH_DBG_ON+x $\} "]$; then

case "\$-" in

$* v * x *)$

_-_lmod_sh_dbg= ' vx'

; ;

$\star v *)$

_-_lmod_sh_dbg=' $v$ '

; ;

$* x *)$

__lmod_sh_dbg=' $x$ '

; ;

esac;

fi;

if [ - n "\$\{__lmod_sh_dbg:- $\}$ " ]; then

set +\$_llmod_sh_dbg;

echo "Shell debugging temporarily silenced: export

$\hookrightarrow$ LMOD_SH_DBG_ON=1 for Lmod's output";

fi;

eval \$(\$LMOD_CMD bash "\$@") \&\& eval

$\hookrightarrow \quad \$\left(\$\left\{L M O D \_S E T T A R G \_C M D:-:\right\}-s \quad s h\right)$;

local_lmod_my_status $=\$$; 


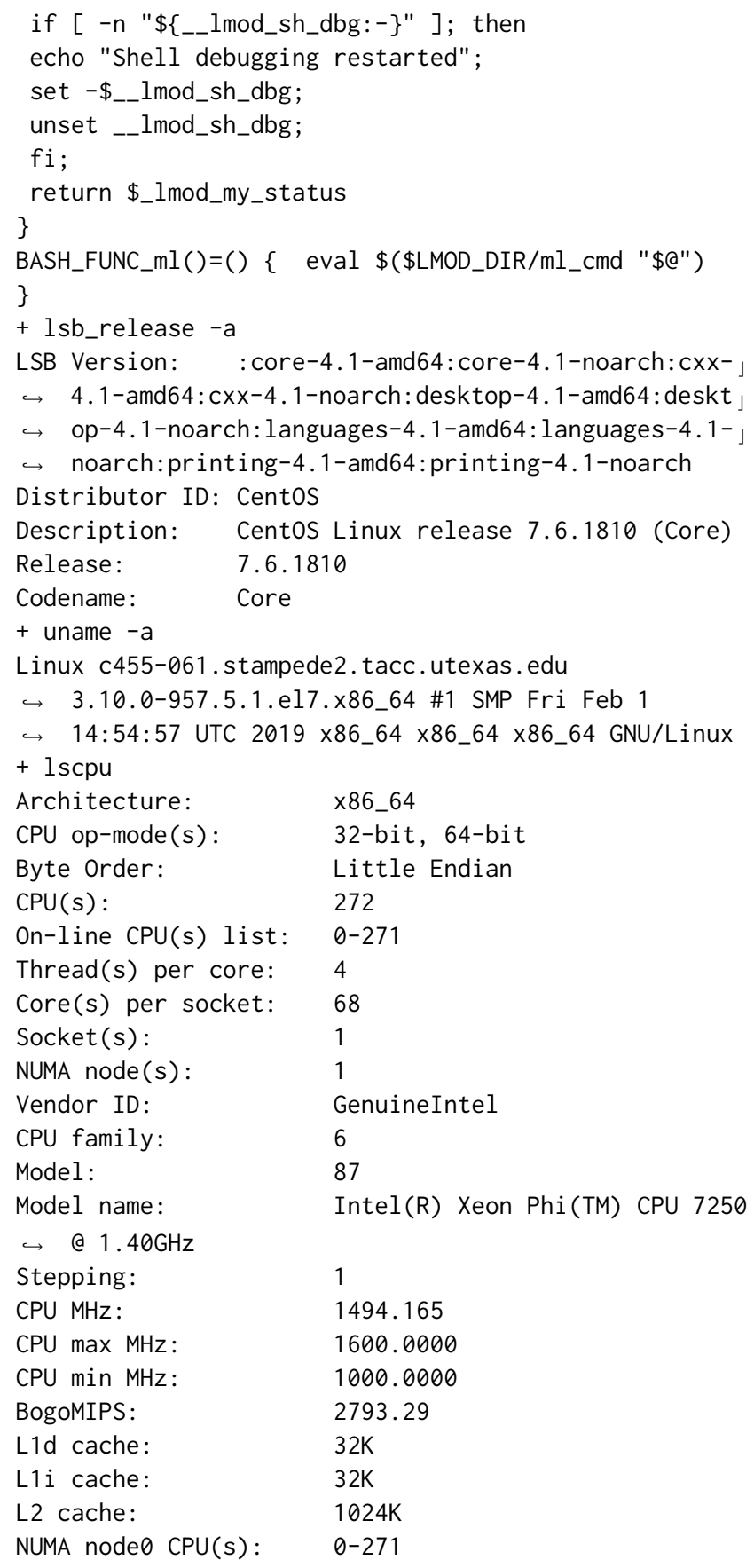

Flags:

fpu vme de pse tsc msr pae mce

$\hookrightarrow$ cx8 apic sep mtrr pge mca cmov pat pse36 clflush

$\hookrightarrow$ dts acpi mmx fxsr sse sse2 ss ht tm pbe syscall nx

$\hookrightarrow$ pdpe1gb rdtscp lm constant_tsc arch_perfmon pebs

$\hookrightarrow$ bts rep_good nopl xtopology nonstop_tsc aperfmperf

$\hookrightarrow$ eagerfpu pni pclmulqdq dtes64 monitor ds_cpl est

$\hookrightarrow \quad$ tm2 ssse3 fma cx16 xtpr pdcm sse4_1 sse4_2 x2apic

$\hookrightarrow$ movbe popcnt tsc_deadline_timer aes xsave avx f16c

$\hookrightarrow$ rdrand lahf_lm abm 3dnowprefetch ring3mwait epb

$\hookrightarrow$ ibrs ibpb fsgsbase tsc_adjust bmi1 avx2 smep bmi2

$\hookrightarrow$ erms avx512f rdseed adx avx512pf avx512er avx512cd

$\hookrightarrow \quad$ xsaveopt dtherm ida arat pln pts spec_ctrl

+ cat /proc/meminfo

MemTotal: $\quad 98698220 \mathrm{kB}$

MemFree: $\quad 88660500 \mathrm{kB}$

MemAvailable: $88227780 \mathrm{kB}$

Buffers: $\quad 0 \mathrm{kB}$

Cached: $\quad 1177244 \mathrm{kB}$

SwapCached: $\quad 0 \mathrm{kB}$

Active: $\quad 151664 \mathrm{kB}$

Inactive: $\quad 1147140 \mathrm{kB}$

Active(anon): $\quad 122740 \mathrm{kB}$

Inactive(anon): $1107700 \mathrm{kB}$

Active(file): $28924 \mathrm{kB}$

Inactive(file): $\quad 39440 \mathrm{kB}$

Unevictable: $\quad 0 \mathrm{kB}$

Mlocked: $\quad 0 \mathrm{kB}$

SwapTotal: $\quad 0 \mathrm{kB}$

SwapFree: $\quad 0 \mathrm{kB}$

Dirty: $\quad 0 \mathrm{kB}$

Writeback: $\quad 0 \mathrm{kB}$

AnonPages: $\quad 121724 \mathrm{kB}$

Mapped: $\quad 74876 \mathrm{kB}$

Shmem: $\quad 1108868 \mathrm{kB}$

Slab: $\quad 4957208 \mathrm{kB}$

SReclaimable: $\quad 633376 \mathrm{kB}$

SUnreclaim: $\quad 4323832 \mathrm{kB}$

KernelStack: $\quad 43088 \mathrm{kB}$

PageTables: $\quad 8012 \mathrm{kB}$

NFS_Unstable: $\quad 0 \mathrm{kB}$

Bounce: $\quad 0 \mathrm{kB}$

WritebackTmp: $\quad 0 \mathrm{kB}$

CommitLimit: $\quad 90802360 \mathrm{kB}$

Committed_AS: 1521944 kB

VmallocTotal: 34359738367 kB

VmallocUsed: $\quad 1585460 \mathrm{kB}$

VmallocChunk: 34357935928 kB

HardwareCorrupted: $\quad 0 \mathrm{kB}$

AnonHugePages: $\quad 38912 \mathrm{kB}$

CmaTotal: $\quad 0 \mathrm{kB}$

CmaFree: $\quad 0 \mathrm{kB}$

HugePages_Total: 0

HugePages_Free: 0

HugePages_Rsvd: $\quad 0$

HugePages_Surp: $\quad 0$

Hugepagesize: $2048 \mathrm{kB}$

DirectMap4k: $1181920 \mathrm{kB}$ 


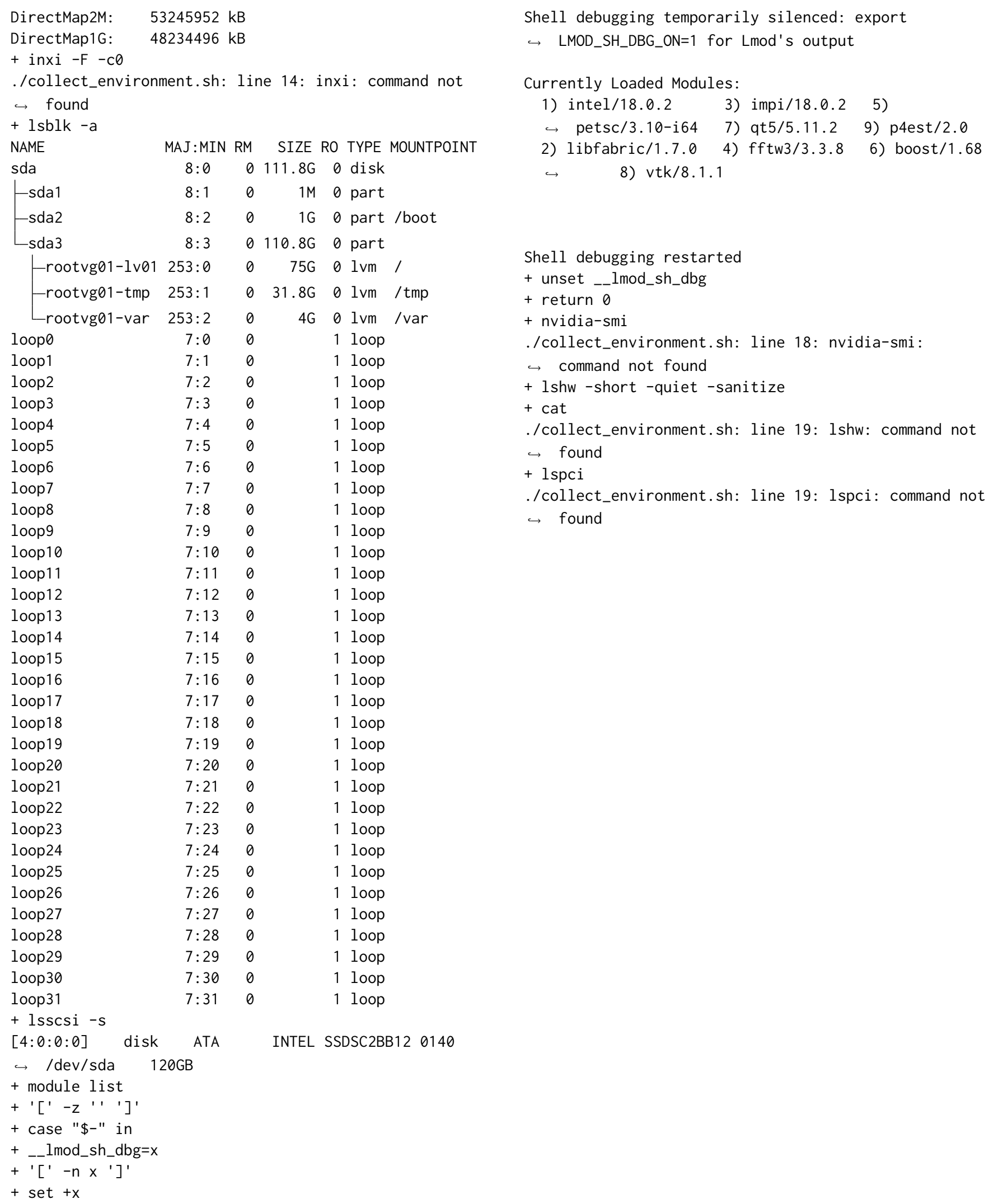

Shell debugging temporarily silenced: export

$\hookrightarrow$ LMOD_SH_DBG_ON=1 for Lmod's output

Currently Loaded Modules: 